Portland State University

PDXScholar

$11-8-1991$

\title{
Stochastic Hydrologic Modeling in Real Time Using a Deterministic Model (Streamflow Synthesis and Reservoir Regulation Model), Time Series Model, and Kalman Filter
}

Philip Kwok Fan Tang

Portland State University

Follow this and additional works at: https://pdxscholar.library.pdx.edu/open_access_etds

Part of the Civil Engineering Commons

Let us know how access to this document benefits you.

\section{Recommended Citation}

Tang, Philip Kwok Fan, "Stochastic Hydrologic Modeling in Real Time Using a Deterministic Model (Streamflow Synthesis and Reservoir Regulation Model), Time Series Model, and Kalman Filter" (1991). Dissertations and Theses. Paper 4580.

https://doi.org/10.15760/etd.6464

This Thesis is brought to you for free and open access. It has been accepted for inclusion in Dissertations and Theses by an authorized administrator of PDXScholar. Please contact us if we can make this document more accessible: pdxscholar@pdx.edu. 
AN ABSTRACT OF THE THESIS OF Philip Kwok Fan Tang for the Master of Science in Civil Engineering presented November 8, 1991.

Title: Stochastic Hydrologic Modeling in Real Time Using a Deterministic Model (Streamflow Synthesis and Reservoir Regulation Model), Time Series Model, and Kalman Filter.

APPROVED BY THE MEMBERS OF THE THESIS COMMITTEE:
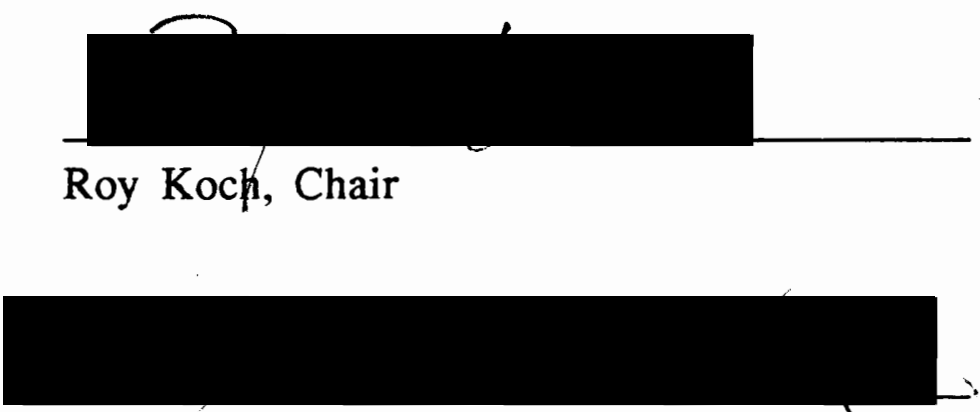

Eugene Enneking

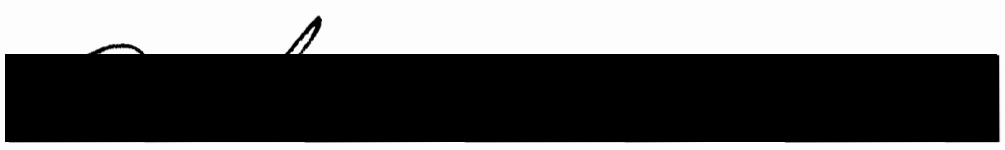

Andrew Fraser

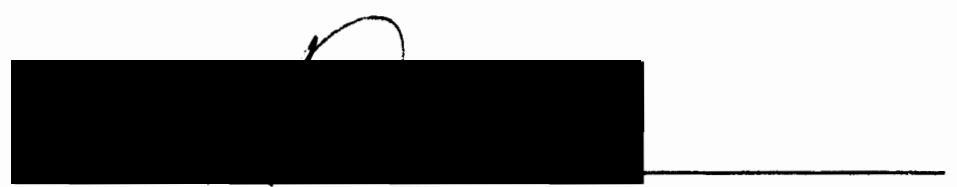

Scott Wells

The basic concepts of hydrologic forecasting using the Streamflow Synthesis And Reservoir Regulation Model of the U.S. Army Corps of Engineers, auto-regressive-moving-average time series models (including Greens' functions, inverse functions, auto- 
covariance Functions, and model estimation algorithm), and the Kalman filter (including state space modeling, system uncertainty, and filter algorithm), were explored. A computational experiment was conducted in which the Kalman filter was applied to update Mehama local basin model. (Mehama is a 227 sq. miles watershed located on the North Santiam River near Salem, Oregon.), a typical SSARR basin model, to streamflow measurements as they became available in simulated real time. Among the candidate AR and ARMA models, an ARMA(1,1) time series model was selected as the bestfit model to represent the residual of the basin model. It was used to augment the streamflow forecasts created by the local basin model in simulated real time. Despite the limitations imposed by the quality of the moisture input forecast and the design and calibration of the basin model, the experiment shows that the new stochastic methods are effective in significantly improving the flood forecast accuracy of the SSARR model. 
STOCHASTIC HYDROLOGIC MODELING IN REAL TIME USING A DETERMINISTIC MODEL (STREAMFLOW SYNTHESIS AND RESERVOIR REGULATION MODEL),

TIME SERIES MODEL, AND

KALMAN FILTER

by

PHILIP KWOK FAN TANG

A thesis submitted in partial fulfillment of the requirement for the degree of

\author{
MASTER OF SCIENCE \\ in \\ CIVIL ENGINEERING
}

Portland State University

1992 


\section{TO THE OFFICE OF GRADUATE STUDIES:}

The members of the Committee approve the thesis of Philip Kwok Fan Tang presented November 8, 1991.

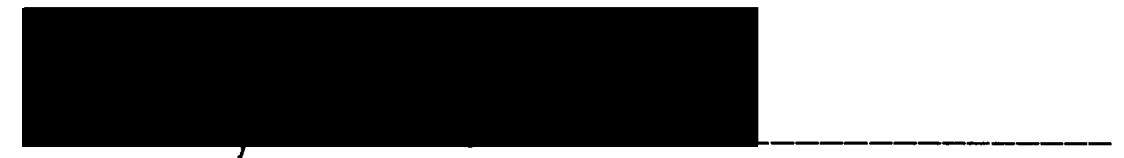

$$
\text { Roy W/Koch, Chair }
$$

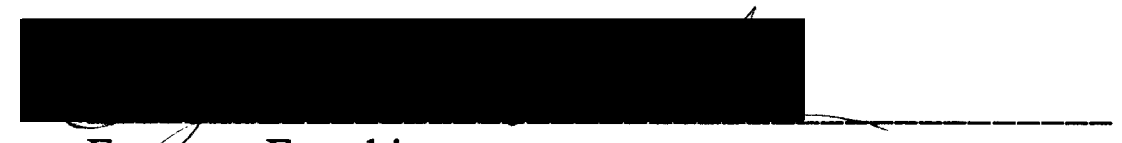

Eugene Enneking

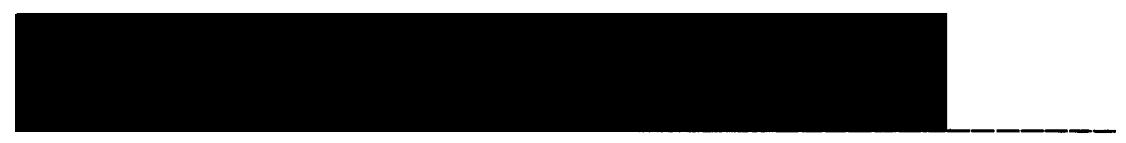

\section{Andrew Fraser}

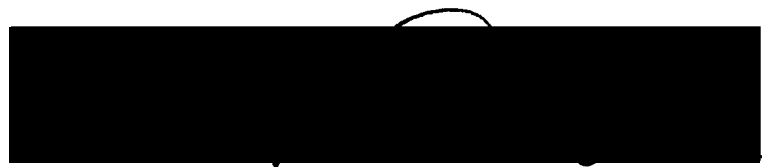

Scott Wells

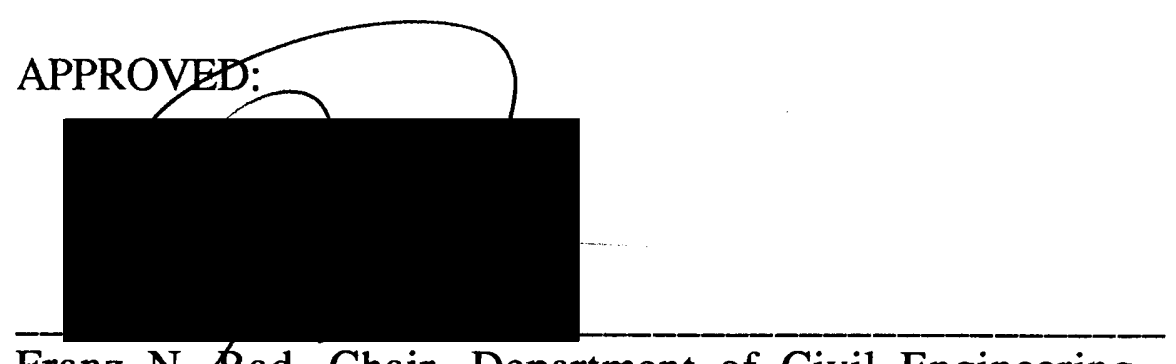

Franz N. Kad, Chair, Department of Civil Engineering

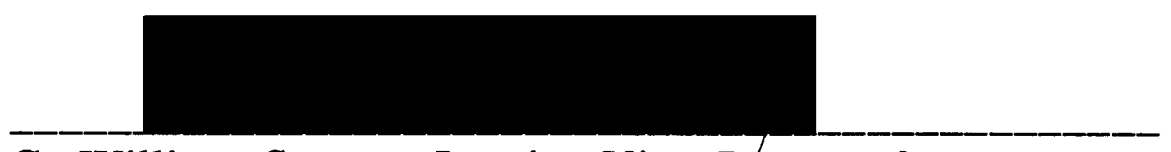

C. William Savery, Interim Vice Provost for

Graduate Studies and Research 


\section{ACKNOWLEDGMENT}

This project was funded in part through a grant from the North Pacific Division, U.S. Army Corps of Engineers. The funding allowed the author to undertake the research covered in this thesis and the development of a data handling program that automates the review and input of hydrometeorological data and operation decisions to the SSARR model.

The author expresses his appreciation to Douglas Speers of the U.S. Army Corps of Engineers, North Pacific Division, who administered the funding for this study, to Ed Davis of the Corps who provided timely SSARR lessons, to the Hydrologists of National Weather Service Northwest River Forecast Center, Dr. Bissell (who provided technical liaison), Mr. Orwig, Mr. Hartman, Mr. Halquist, Mr. Holbrook, Mr. Garen, Mr. Mathison, Mr. Pasteris, and Mr. Willoughby for their technical support, advice, and friendships, to Dr. Koch of Portland State University, the author's academic advisor, for his teaching, guidance, and unfailing moral support. 


\section{TABLE OF CONTENTS}

PAGE

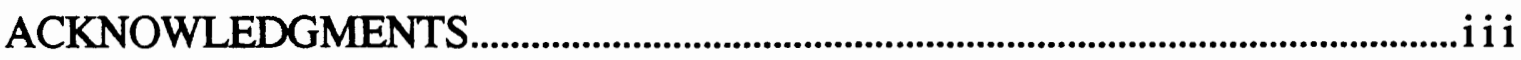

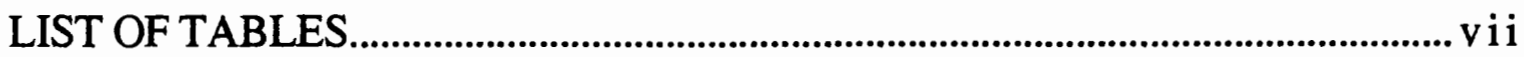

LIST OF FIGURES ..............................................................................................

\section{CHAPTER}

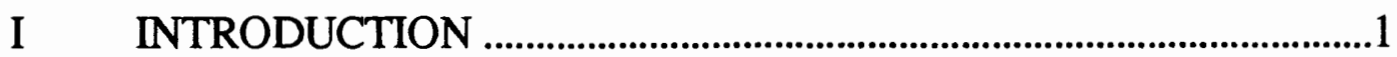

Background ............................................................................. 1

Problem Definition .............................................................. 2

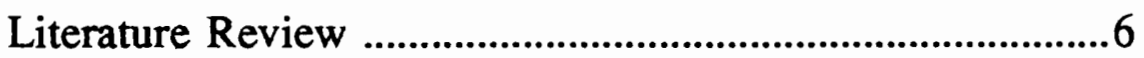

II AN OVERVIEW OF COMPUTER MODELING .................................... 15

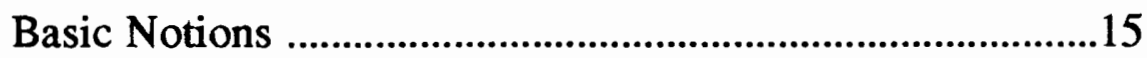

The Cognitive Value of a Computer Model..................17

A Real World System, an Imperfect Measurement, and an Imperfect Computer Model ...............................18

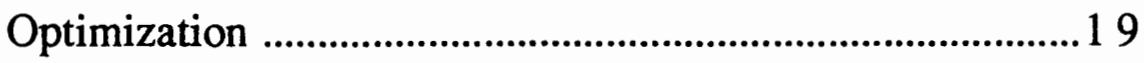

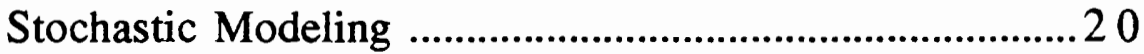

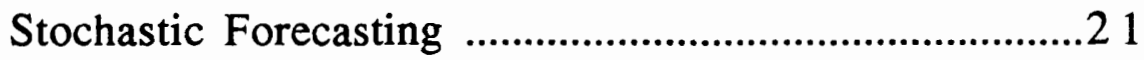

III SSARR MEHAMA LOCAL BASIN MODEL ....................................... 23

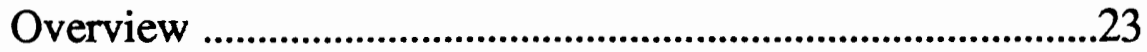

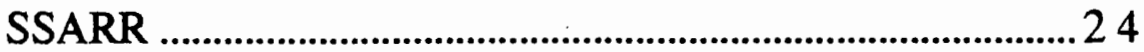


"Measuring" Runoff from Mehama Local

Basin .25

Modeling Moisture Input to Mehama Local

Basin .27

Modeling the Runoff of Mehama Local Basin .33

IV TIME SERIES MODELING 40

Introduction 40

White Noise 41

Porte Manteau Test for White Noise 43

Basic Model Forms .43

Basic Mathematical Characteristics of a

Time Series Model .46

Green's Functions .47

Inverse Functions 49

Auto-Covariance Functions ….......................................52

Model Estimation Techniques .......................................55

V STATE SPACE MODELING .........................................................64

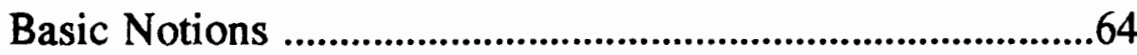

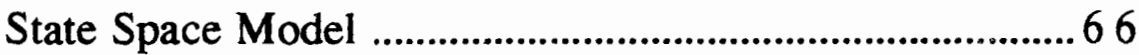

Selecting Model States ......................................................68

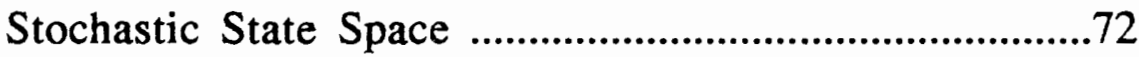

VI KALMAN FILTER _...................................................................... 73

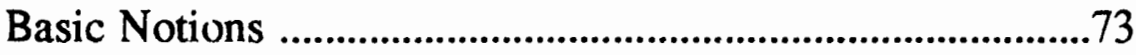

Three Kinds of State Estimation Problems...............76

Filtering in One-Dimension:

An Illustration 
Kalman Filter Algorithm in One -Dimension

Filtering in Multi-Dimensions -

The General Problem

Kalman Filter Algorithm in

Multi-Dimensions

The Sequence of Events in a Real Time

Modeling/Monitoring Scenario

VII COMPUTATIONAL EXPERIMENT

Overview-Basic Scheme and Assumptions

of the Experiment

Data .98

Model Residual (Noise) 102

Estimating a Time Series Model 103

Time Series Model Augmenting Mehama

Local Basin Model

Mehama Local Basin Model in State Space

Artificial Design of System Noise

Covariance Matrix

Performance Evaluation 


\section{LIST OF TABLES}

TABLE

PAGE

I Statistical Characteristics of the Residual

Time Series, Mehama Local Basin Model .104

II Time Series Estimation Results..........................................104

III Characteristics of Candidate Models .................................106 


\section{LIST OF FIGURES}

1. Schematic diagram of a local basin ....................................26

2. SSARR Model Schematic Flow Chart .........................................28

3. Discrete Time Series ..............................................................41

4. Three Applications of Kalman Filter .....................................77

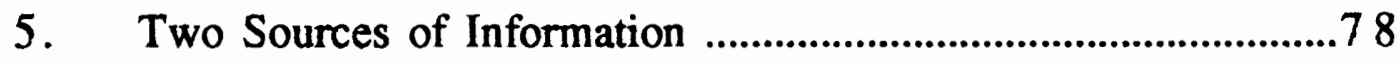

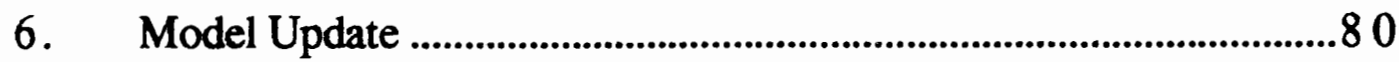

7. Change of State Variance Through Time ..............................82

8. Moisture Input to Mehama Local Basin 0000 hour

October 1 to 2400 hour December 31, 1982 ........................99

9. "Measured" Streamflow of Mehama Local Basin 0000 hour October 1 to 2400 hour December 31, 1982 .............99

10. Mehama Local Basin Model Error 0000 hour October 1 to 2400 hour December 31, 1982 ..........100

11. Residual-Squared Time Series, 0000 hour October 1 to 2400 hour December 31, 1982 ..............................................1 01

12. Comparison of Performance based on root mean square forecast errors 


\section{CHAPTER I}

\section{INTRODUCTION}

\section{BACKGROUND}

In recent decades, government agencies have been increasingly interested in the systematic monitoring and control of the hydrologic systems of the nation. One remarkable example is the establishment of Northwest River Forecast Center in Portland, Oregon (RFC). At the center, the numerous components of the water resource system of the Columbia Basin such as reservoirs, river reaches, and watersheds are individually modeled and monitored in real time using state-of-the-art hydrometeorological telemetry, computer database and computer models. The Streamflow Synthesis And Reservoir Regulation (SSARR) model is used by the RFC to simulate the streamflow associated with each reservoir, river reach, or watershed in the mainframe computer. The entire Columbia Basin is simulated by a network of interconnected SSARR models.

The primary input to the hydrologic models is moisture in the form of rainfall and snowmelt. Rainfall is estimated from measurements at rain gauges; snowmelt is estimated from snow surveys and temperature data. For making streamflow forecast, the moisture input to the models may also be derived from weather forecasts. 
To simulate the Columbia Basin, the individual hydrologic models are schematically connected such that the outflow of one hydrologic model is computationally the inflow of another hydrologic model. Hydrologists are thus able to make routine hydrologic forecasts and provide timely management recommendations to the various agencies for purposes such as flood warnings, flood control, flood mitigation, water supply, hydroelectric power, irrigation, navigation, wildlife preservation, and recreation.

The Mehama Local Basin is one of the many hydrologic systems being monitored and modeled by the RFC and is the subject of the computational experiments conducted in this thesis. It is located on the North Santiam River near Salem, Oregon, between the Big Cliff and Mehama gaging stations. The North Santiam River is a tributary to the Willamette River which is in turn a tributary to the Columbia River. The 227 sq. mile watershed has long been modeled and monitored by the RFC using the SSARR model.

\section{PROBLEM DEFINITION}

A computer model and its real world counterpart do not always agree. That is generally due to the imperfect model representation and the imperfect measurements of the real world system. In the real time modeling and monitoring of a watershed using SSARR, the forecast streamflow generated by a model and the streamflow later measured rarely agree exactly. This "noise" in the hydrologic model and measurement system is due to the following: 
1) SSARR represents only the dominant characteristics of the watershed by fitting an pre-determined conceptual model structure to an historic data set of a finite duration.

2) The rainfall and snow melt for a watershed are estimated based on field measurements at discrete locations and time-intervals. The quality of these estimates are affected by the spatial and temporal heterogeneity of the climatic phenomena, the intrinsic error in the measurement techniques, and the intrinsic error in the instruments.

3) In the case of Mehama Local Basin, the "measured" streamflow at the basin outlet is not directly measured; it is computationally deduced from streamflow measured at neighboring gages using a SSARR river routing model. This deduction introduces additional error to the "measured" streamflow.

As the noisy modeling/monitoring system is propagated through each time step, error is accumulated incrementally in the memory of the model. If the model is not routinely corrected, it will slowly drift away from the actual behavior of the system and begin to yield increasingly inaccurate streamflow forecasts. This real 
time updating of the model memory to the latest measurement is known as filtering.

The model and the measurement are the two sources of information in this forecasting system. The model, though containing noise, provides a conceptual structure for relating the dominant characteristics of the watershed. The measurement, though containing noise, is a direct representation of the real world system at certain locations and times. At the time of this study, the SSARR Mehama Local Basin Model was updated manually by RFC hydrologists or by a simplistic computational algorithm which involves multiplying some model parameters by a single factor on a trial-and-error-basis. The purposes of this study are to demonstrate the use of Kalman filter as a automatic stochastic update method and to demonstrate the use of a time series model that utilizes the pattern in the model noise to make better forecasts.

This thesis will illustrate how the stochastic modeling methods can be applied to a SSARR model based on an assumed noise structure in the model, measurements of moisture input, and measurements of streamflow. To limit the scope of this research in proportion to the available research funding, noise in the streamflow measurements will be assumed small compared to the noise in the model representation and the noise in moisture input. Hence, the model is said to be performing satisfactorily when its forecast streamflow value is close to the measured streamflow value. Also, when using the Kalman filter to update the model based 
on the latest streamflow measurement, the memory of the model and the moisture input are both adjusted such that the model streamflow will be almost equal to the measured streamflow. The specific tasks undertaken in this study are:

1. Investigate the mathematical characteristics of the SSARR model and its application to the Mehama Local Basin.

2. Explore the basic concepts of time series analysis, state space modeling, and Kalman filter that are pertinent to real time hydrologic modeling.

3. Fit a time series model to the residual time series (i.e. noise) between the model forecast and the measurement of Mehama Local Basin, for the historic data of October, November, and December of 1984. Demonstrate the use of this time series model in enhancing the model forecast in simulated real time through the months of October, November, and December of 1984.

4. Convert SSARR Mehama Local Basin Model into state space form, so that the concept and algorithm of the Kalman filter can be readily applied.

5. Demonstrate the use of Kalman filter in the routine updating of the SSARR Mehama Local Basin Model in a 
simulated real time monitoring scenario through the months of October, September, and November of 1984.

\section{LITERATURE REVIEW}

Among the many scientific and engineering pursuits, the hydrologic cycle has been given considerable attention due to the vital and paramount importance of water to human affairs and the biosphere in general. Dunne and Leopold (1983) presented systematically the basic scientific and engineering principles governing the behavior of water in the environment along with a comprehensive treatment of the various water problems faced by engineers and planners. Numerous mathematical models have been created for the various components of the hydrologic cycle. Haan et al (1982) have provided general insight into the mathematical modeling of the various sub-systems in the hydrologic cycle, including the applications of both the deterministic and stochastic methods.

The uses of stochastic methods, as opposed to the more traditional deterministic methods, are sometimes viewed as controversial. As an opponent of the extensive use of stochastic methods in Hydrology, Klemes (1982) argued that mathematical modeling should increase human understanding of the hydrologic system and any effort that is outside of this purpose are of less importance. However, addressing the pragmatic issues faced by modelers, Shaw (1984) used the investigation of a dripping faucet as 
a chaotic system to illustrate the fundamental characteristics of the modeling process. In the introduction, Shaw wrote

We live in a whirl of moving structures, swept by social, economic, and personal currents whose dominant theme is one of unpredictability. Yet laws, constraints of some sort seem to be operating, as evinced by our ability to function. The central issue of physics, that of predictability, is in fact addressed as a practical matter by each newborn infant: How do we construct a model from a stream of experimental data which we have not seen before? How do we use the model to make predictions? What are the limits of our predictive ability? Simple experiments, as well as the experience of daily living, still have much to teach us.

The uses of stochastic modeling methods have been wide spread among the scientific and engineering practices in general. The advent of computer technology has made the implementation of stochastic methods readily practicable. Among the many excellent comprehensive treatments, Maybeck (1979) presented the concepts of stochastic dynamic system and the Kalman filter. Its discussions on the propagation of a dynamic system through time, the mechanism of filtering, and the characteristics of white noise are of particular relevance to hydrologic modeling. Likewise, Gelb et al (1974) presented the concepts of estimation using the Kalman filter, with a emphasis on the computational detail and implementation problems. Gelb et al also presented the concepts of state space modeling, a prerequisite for using the Kalman filter. Kalman filter is a solution to the problem of optimal estimation. Gelb (1974), referring to real time modeling in general, wrote 
An optimal estimator is a computational algorithm that processes measurement to deduce a minimum error estimate of the state of a system by utilizing: knowledge of system and measurement dynamics, assumed statistics of system noises and measurement errors, and initial condition information.

The use of the Kalman filter is widespread in the science and industry to process real-time signals for supporting decision making. For example Schlee, Standish, and Toda (1967) discussed the divergence of the Kalman filter in the context of a space navigation system. In the system, the Kalman filter was used in real time to compute differential corrections to the location of a landmark and the position and velocity of a spacecraft. Another category of popular stochastic methods is time series modeling, a "black-box" approach to derive model forms from time series data. An excellent example of a comprehensive treatment is Pandit and Wu (1983) which discussed the basic concepts of time series analysis in terms of the Green's functions, inverse functions, and the auto-covariance functions and provided detailed derivation and practical computational algorithms for estimating time series models such as the $\operatorname{ARMA}(1,1), \operatorname{ARMA}(2,1), \operatorname{ARMA}(2,2)$, and $\operatorname{ARMA}(3,1)$ models by mathematical inference.

Parallel to the development in the general scientific and engineering practices, stochastic modeling methods such as the Kalman filter and time series analysis in Hydrology have become widely accepted in the recent years. At National Weather Service 
Northwest River Forecast Center (Portland), Koch (1985) presented a lecture series on the subject of optimal estimation in hydrology which stressed the uses of stochastic methods. Anderson, Burt, et al (1985) reviewed and reported the general concepts and practices in the hydrologic field; the concepts of modeling and the Kalman Filter were reviewed in considerable details. Salas et. al. (1985) is a textbook devoted to the subject of fitting hydrologic time series with the various forms of time series models. Clearly defining the black-box philosophy of time series modeling, Salas et. al. (1985) wrote, "The exact mathematical models of hydrologic time series are never known. The inferred population models are only approximations. The exact model parameters are also never known in hydrology since they must be estimated from limited data." Szollosi-Nagy (1976) explored the basic concepts of deterministic and stochastic state space models in discrete and continuous space and demonstrated their use to water resources system.

There are numerous cases in which the stochastic methods have been successfully applied: Lettenmaier and Burges (1976) concisely explored the basic concepts of Kalman filter and its application to the BOD and DO in stream water as they are governed by the Streeter-Phelps Equations. Additionally, brief discussions were made on applying Kalman filter to measurement system design, analysis of requirement for model building and assessment of models, extending the utility of existing data, and calibration of models. Moore (1973) designed a water quality monitoring system by using Kalman filter to determine the states to be measured and the 
temporal and spatial frequencies of sampling. That was done by representing the eco-system with a state space model and deriving the minimum measurement requirement that allows state estimation of acceptable uncertainty at minimum operation cost. Jones and Koch (1985) used Kalman Filter in flood forecasting at the Toutle and Cowlitz Rivers in the State of Washington. Streamflow was modeled by the Simplified Muskingum Routing. The nonlinear equation was first linearized by Taylor expansion and then rewritten in state space form. Variance in the measurement was derived by analyzing the model structure and the stage versus discharge relationship; variance in the system model were derived from comparing the model streamflow with the measured streamflow for an actual storm. Based on an actual storm event, the study showed that flood forecast accuracy was improved by the application of the Kalman filter. Hains (1988) applied Kalman filter to estimate the states of a rainfall-runoff model. He observed that water mass is not conserved through the filtering process. That is, the filter computationally added/subtracted water to/from the memory of the model in order to match the measurement. Refsgaard, Rosebjerg, and Markussen (1983) used Kalman filter to update a deterministic lumped conceptual rainfall-runoff model known as NAM. NAM was rewritten into state space form; the states are the water content in surface storage, water content in lower zone storage, water content in groundwater storage, water content in routing reservoir no. 1, water content in routing reservoir no. 2 , time constant in routing, and the parameter in an overland flow equation. Sources of 
uncertainty in the modeling system were grouped in four categories: the error in input data to model, the error in measurement of output from nature, the error in model structure, and the non-optimal values of model parameters. The first two categories were accounted for quantitatively; the last two categories were accounted for in a "heuristic manner". Analysis of uncertainty in the study showed that the uncertainty in rainfall input is "significantly predominant for the resulting uncertainty of simulated runoff values." The study showed that forecast accuracy was gained by using Kalman filter to update the model. Ngan and Russell (1986) used Kalman filter with a streamflow forecast model that was in the form of a ARMAX time series model. The model coefficients of the ARMAX model were selected as states of the system and were estimated by Kalman filter. The ARMAX model itself was conveyed by the observation equation. Variances in measurement and model were estimated by a method based on maximum likelihood. Data to the system was first transformed before filtering, allowing the estimation of variance in measurement to be approximately constant. The study showed how "art" is involved in the design of a successful filter. One-day ahead streamflow forecast was made for Fraser River in British Columbia. Moll (1983) used Kalman filter in a flood forecast model which is based on a differential equation governing convection and diffusion with constant parameters. The partial equation was solved numerically using finite difference approximations; ARIMA time series model was used as one of the boundary conditions. Kalman Filter was used to estimate the flow at 
the "grid points" in the finite difference scheme. Ambrus and Szollosi-Nagy (1983) used ARMAX time series models in adaptive mode using Kalman filter which continually updated the model parameters in real time; the model was for estimating hourly expected water demands necessary in making efficient water allotment decision. The water demand time series contains randomness; therefore deterministic method alone would not suffice. The deterministic pattern was modeled by a Fourier Series; the random component was modeled by an ARMAX time series model. The Fourier + ARMAX model (of 2nd or 3rd order) was then written in state space form and Kalman filter was applied; lagged values of water demand random residual series were chosen as model states. Prediction error was computed based on historic data. Due to the online model estimation by Kalman filter, initial estimation of the time series model was avoided. Tested on actual historic data, the experiment showed that forecast accuracy was gained by adopting the stochastic approach using the time series model and Kalman filter.

The wide-acceptance of the stochastic methods may be due to the fact that a deterministic model often falls short in representing the entirety of the observed phenomenon, unless the observed phenomenon is remarkably simple. That is, a deterministic model and its real life counterpart do not always agree. Given a real time computer model and a measurement that do not agree, how does one arrive at an understanding of the true state of the system? This balancing act between the modeled and the measured is the charter 
of optimal estimation. As demonstrated in the aforementioned studies, the discrepancy between the deterministic model and the observation are oftentimes successfully resolved by the stochastic methods.

This thesis further explores the benefits of the stochastic methods, in this case, Kalman filter and time series modeling, as applied in the practice of real-time operational hydrology at the RFC. In the day-to-day operation of the RFC where real-time computer models and advanced hydrometeorological telemetry are used, timely and accurate hydrologic forecast is imperative. One routine and fundamental challenge faced by the hydrologists at the RFC is to make the SSARR models and the actual observations agree, as discussed by Bissell and Orwig (1984). Each SSARR lumped-basin model in operation at the RFC is routinely updated by running the model in "backup" mode in which the hydrologic variables in the model are adjusted manually such that the basin outflow computed by the model will agree with the observed outflow. The manual adjustment involves changing the amount of moisture input by a multiplicative factor derived on a trial-and-error basis; it also involves other options such as changing the soil moisture index, the melt rate of snow, and snow line elevation. The adjustability of these model variables varies differently through time within a forecast season which starts on December 1 and ends on June 1 of each year. Ultimately, this manual adjustment is based on the judgment of the operation hydrologist(s) performing the model backup. This thesis shows how SSARR, an deterministic model, can 
be augmented by the stochastic methods and how forecast accuracy is thereby improved. Unlike the manual methods discussed by Bissell and Orwig (1984) which relies heavily on engineering judgment, this thesis demonstrates the uses of the Kalman filter and a time series model which are automatic, mathematical, and ready to be integrated into the computer codes of SSARR for routine operation. 
CHAPTER II

\section{AN OVERVIEW OF COMPUTER MODELING}

\section{BASIC NOTIONS}

This chapter explores the characteristics of computer modeling pertinent in further defining the technical perspective of this thesis. The concepts and terminology discussed herein are general and wide spread among publications on modeling. Examples are textbooks such as Haan, et al (1982), Anderson, et al (1985), Gelb (1984), Shaw (1984), Maybeck (1979, and Salas, et al (1980).

To model a real world system is to represent the system in a mathematical form. That is done by analyzing the observed input and output of the real world system. Normally, the course of analyses are guided by apriori knowledge of the system. The modeler's knowledge of the system should in turn increase as a result of the modeling effort. A computer model is a computational "simile" of a real world system. The model user generally obtains a feeling of the real world system by studying the model.

A record of the system signal is achieved by measurement. In general, the system signal is multivariate. For example, a watershed can be measured in terms of its streamflow at various points, its storage of water underground, and its ever changing soil moisture which affects infiltration, among many possible variables. The 
modeler usually chooses to measure a system using a minimal number of variables to be cost-effective. The choice is dictated by the purpose of modeling. For example to model the flooding downstream from a small watershed, one would choose to measure only the streamflow at the mouth of the watershed and a few other variables that strongly affect the streamflow.

Models are almost always considerably less complex than their real world counterpart. This may be due to the limitations in funding or a lack of understanding of the nature of the system. However, a simplistic model is easier to understand. It can provide a better tool for the model user in understanding the dominant characteristics of the real system. Whether this tool is effective or not depends entirely on the quality of the model.

To a modeler, a real world system can be viewed as a body of information which gives out a signal in the domain of space and time. A model is a computational algorithm that generates signal in a fashion similar to that of the observed real world system. Modeling is itself an exercise of information processing. Its purpose is to investigate and predict. The processing of information (i.e. modeling) is made more complicated when one considers the involvement of uncertainty in the flow of information. Firstly, the frequency of measurement in the domains of space and time would dictate whether enough of the systems signal is sampled so that the system is adequately represented in the recorded information. One's understanding of the system is limited by the quality of the measurement. Secondly, a computer model generally represents only 
the dominant characteristics reflected in the measured signal. Information is inevitably corrupted, first by the measurement process, second by the modeling process. This corruption of information is manifested as disagreement between the simulation model and the real world system. This uncertainty corrupting the flow of information is also called noise. Noise must be studied and described by stochastic methods. Using statistics and computational tools, the model could, in some instances, characterize and utilize the model noise itself as a source of information which in turn enhances the quality of the model. In essence, one of the goals of this thesis is to enhance the model using its noise.

\section{THE COGNITIVE VALUE OF A COMPUTER MODEL}

In recent years, computer technology has allowed the formation of many databases, but data by themselves do not necessarily provide understanding for the human mind. This is particularly true when the data are from a complex system. Cognitive understanding of the data can be derived by modeling the data; that is, by fitting the data into a conceptual framework which provides an explanation. In the case of SSARR, this "cognitive value $^{n}$ is crucial and essential for the hydrologists to acquire a timely and systematic understanding of the huge quantity of hydrometeorological data on the complex hydrologic systems made available by state of the art telemetric equipment.

Cognition is a human activity, but it can be greatly assisted by the use of computer. By fitting a computational structure of flow 
components (i.e., surface flow, subsurface flow, baseflow, and deep baseflow) to a time series of observed streamflow of a watershed, the hydrologist acquires insight as to how moisture input may have been distributed, stored, and released as streamflow. The hydrologist acquires further insight into the hydrologic system by changing the values of some of the model parameters such as the moisture input and precedent soil moisture index and running the model to see how the streamflow characteristics are affected.

\section{A REAL WORLD SYSTEM, AN IMPERFECT MEASUREMENT, AND AN IMPERFECT COMPUTER MODEL}

A real world system is generally composed of a huge number of sub-systems. From an operational standpoint, it is not costeffective and may not even be possible to fully investigate each subsystem and represent it with a mathematical form. Likewise, it is generally neither cost-effective nor possible to completely represent a real world system by a computer model, unless the particular real world system is remarkably simple.

A real system is generally continuous and heterogeneous in space and time while measurements are usually discrete in time and space. From an operational standpoint, it is generally neither costeffective nor possible to completely measure a real word system continuously in time and space. A hydrologic system (a reservoir, river reach, or a watershed) definitely fits into this general category of real world system in that it is continuous and heterogeneous in space and time and it consists of large number of 
subsystems. Consider a watershed where the precipitation characteristics, vegetation cover, surface and subsurface soil and rock characteristics, drainage characteristics, and topography all vary continuously and heterogeneously in both space and time. A huge number of subsystems will be involved if one was to exhaustively represent its streamflow generation behavior.

\section{OPTIMIZATION}

Optimization is a basic modeling tool. In essence, to model a physical system is to find a mathematical form that optimally represents the physical system. Generally, the criteria of optimality are always modified by the constraints in the time and funding available to create and operate the model. In other words, parsimony as an engineering concept is almost always a built-in modifier to the criteria of model optimality.

The basic idea of optimization can be summarized in this working template (Rekalaitis, et al, 1983) :

Find the values of a set of variables that will maximize (or minimize) a criterion function by adjusting the variables within a certain range of values. For example, to estimate a time series model given a time series data set, one need to find the form and the values of the model parameters that will minimize the model residual sum of square function by adjusting the form and the parameters within the range of available forms and parameter values confined by the conditions of stationary, invertibility, white noise residual, and parsimony. 
Model calibration is an exercise of optimization. At the RFC, hydrologists adjust the parameters of the SSARR model within reasonable ranges of values to find the best fit to a certain hydrologic system (e.g. Mehama Local Basin). Here, the criterion of best fit is based on engineering judgment. Given a differentiable mathematical function, maxima and/or optima can be derived analytically.

\section{STOCHASTIC MODELING}

The origin of the word "stochastic" connotes the shooting of an arrow toward a target. Despite the intent of the archer, there is a significant room for chance. A stochastic model has two components: a deterministic and a random component. Each component can be thought of and treated as a sub-model. The deterministic sub-model represents the physics of the system and it can be described in a mathematical form relating the inputs and the outputs of the system (e.g. a system of differential or difference equations). The random sub-model represents the statistics of the system and it can be described by statistical models. If the stochastic component is completely random, this random sub-model is a white-noise model. A white noise model generates a series of values that do not correlate with one another in time.

From an operational stand point, the SSARR model has been treated as strictly deterministic. That is, stochastic methods have not been used in the operation of SSARR. This thesis is an attempt to deal with the uncertainty of the SSARR Mehama Local Basin Model. 
The model noise, i.e. discrepancy between the modeled and the measured streamflow, is studied and analyzed using time series analysis and the Kalman filter.

\section{STOCHASTIC FORECASTING}

A computer model becomes stochastic once noise is considered. Both the model and the measurement are trying to convey information about the real system, but the information is noisy. Thus the likelihood of a result from the computer model conveying the "true information" of the real system is based on the probability distribution $P_{C}\left(I_{c, n}\right)$ while for the case of measurement it is $\mathrm{P}_{\mathrm{m}}\left(\mathrm{I}_{\mathrm{m}, \mathrm{n}}\right)$. I denotes information. The subscript $\mathrm{n}$ denotes a certain time step; $\mathrm{m}$, the measurement; and $\mathrm{c}$, the computer model. The modeler needs to combine the information given in $P_{c}\left(I_{c, n}\right)$ and $\mathrm{P}_{\mathrm{m}}\left(\mathrm{I}_{\mathrm{m}, \mathrm{n}}\right)$ to arrive at an optimum understanding of the system. This optimum understanding can be achieved computationally by applying the Kalman filter to update the computation model.

The problem of stochastic forecasting can be best explained in terms of conditional probabilities. Given the past information on a system made available by a real time computer simulation model and a real time measurement, how likely is it that one can predict the behavior of the system in the future? In other words, $I m, n+1$ is linked to $I_{c, n}$ and $I_{m, n}$ by the conditional probability function $P\left(I_{c, n+1} \mid I_{c, n}, I_{m, n}\right)$ where $I_{c, n}$ is the information of the system from the computer model at time step $n, I m, n$ is the information of the system from the measurement at time step $n$, and $I m, n+1$ is the 
information of the system as predicted by the computer model for time step $n+1$. The quality of a forecast depends on using the information from the model and measurement such that $P\left(I_{c}, n+1 \quad\right.$ $\left.I_{c, n}, I_{m, n}\right)$ will have the minimum variance possible. The purpose of this thesis is to demonstrate how this can be achieved by applying time series modeling and the Kalman filter. 


\section{CHAPTER III}

\section{SSARR MEHAMA LOCAL BASIN MODEL}

\section{OVERVIEW}

The mathematical structure of the SSARR model is described in this chapter. The concepts and computer programs of the SSARR model were developed and are continually updated by the U.S. Army Corps of Engineers, North Pacific Division. The following discussion is limited to the concepts of the watershed model. The complete and in depth discussion of the theory and use of SSARR is found in the User Manual, SSARR Model (U.S. Army Corps of Engineers, 1985).

The SSARR Basin Model belongs to a category of hydrologic model known as "rainfall-runoff" models which take moisture in the form of snowmelt and rainfall as input and create streamflow as output. There are three types of rainfall-runoff models: distributed physically based models, lumped conceptual models, and black-box models. Distributed physically based models are based on mass, energy, and/or momentum considerations in three dimensional space. Lumped conceptual models are based on simplistic, quasi-physical concepts involving inter-connected storages and budgeting procedures that maintain conservation of mass through discrete space and time. Black-box models are based on mathematical concepts, such as those from statistics and system theory, that 
relate precipitation as input and runoff as output. However, no attempt is made to determine the hydrological mechanism behind the mathematics. The SSARR basin model is a lumped conceptual model.

\section{SSARR}

Each SSARR model is a discrete conceptual deterministic real time model. SSARR runs on discrete time steps, as opposed to continuous time. Thus it is readily implementable on a digital computer. The time steps can be of lengths 6-hour, 12-hour, 24hours, etc. The model is conceptual in the sense that the simulation of each hydrologic system (e.g., watershed, river reach, or reservoir) is based on an apriori conceptual structure. This conceptual structure is a computational scheme and is not an exhaustive representation of the physics of the real world system. This computational scheme however does convey a rationalized explanation of the general movement of water through a hydrologic system. In the case of Mehama Local Basin, the computational scheme of the SSARR model distributes moisture input among several flow components with different of response rates: surface flow is the fastest; subsurface flow is slower; and baseflow is the slowest. This distribution of moisture input is carried out by three splitting functions. The soil moisture index distributes between evapotranspiration and runoff; the baseflow infiltration index separates between baseflow and the flow through the sub-surface and surface; surface and sub-surface separation curve separates between surface and sub-surface flow. Furthermore, each of the 
three flow components generates runoff based on a routing algorithm which controls the time-response characteristics. The routing algorithm used in SSARR is known as the linear cascaded reservoirs algorithm (LCRA). This algorithm simulates runoff generation by draining the distributed moisture input through a series of cascaded reservoirs where the outflow of each reservoir is linearly proportional to its storage. For each time steps, the watershed runoff is the sum of the water released from each of the three flow components. This chapter will concentrate on the structure and concepts of the SSARR watershed model. The reservoir model and river reach model, while in some ways similar to the watershed model, will not be discussed.

The latest SSARR watershed model structure has provisions for modeling the flow of water in a watershed as surface flow, subsurface flow, baseflow, and deep baseflow. Each of these flow components is represented by a system of cascaded linear reservoirs. Deep baseflow was not used by RFC in modeling Mehama Local Basin. Thus the following description will not include deep baseflow. However, the concepts discussed in this thesis are also applicable to a SSARR watershed model that has a deep baseflow component.

\section{"MEASURING" RUNOFFFROM MEHAMA LOCAL BASIN}

The Mehama local basin is an actual watershed located in the North Santiam watershed between the stream gage below Big Cliff Dam and Mehama stream gage (see Figure 1). While this local basin is 
a real physical system, its runoff is not directly measured. Instead, the runoff "measurement" is deduced from the two stream gages. This deduction makes use of a SSARR river routing model which is based on the cascaded linear reservoirs algorithm. The runoff from the Mehama Local Basin is computed based on this equation :

$$
M L B=M G O-R R[B C G I]
$$

where MLB is runoff from Mehama local basin; MGO is Mehama gaged outflow; BCGI is the Big Cliff gaged inflow; RR[BCGI] is Big Cliff gaged inflow routed through the river reach between Mehama stream gage and Big Cliff stream gage using a SSARR river routine model.

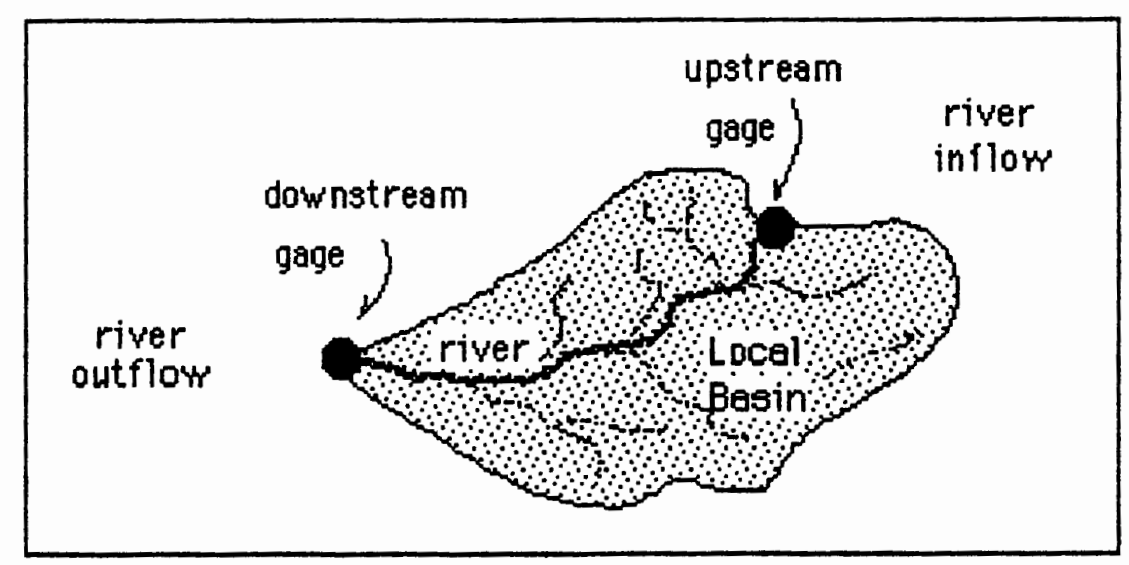

Figure 1. Schematic diagram of a local basin.

A SSARR watershed model was calibrated to an historic data set of runoff from Mehama local basin during several floods. This historic data set was generated by $\mathrm{Eq} 3.1$. In the routine monitoring 
and modeling of the local basin, this equation is used to create the "measured" runoff from the local basin.

When measurement is conducted directly without using any deduction, noise is caused by instrument error and measurement errors in gage height, point velocity, and stage-discharge relationships. Since the "measured" runoff from Mehama Local Basin is computed, it contains both noise due to the measurement method and model noise. In this thesis, to simplify the problem, the noise in this deduced runoff is assumed small in comparison to the modeled runoff. The quantitative comparison between the noise in this deduced runoff and the modeled runoff would be an interesting subject for future studies.

\section{MODELING MOISTURE INPUT TO MEHAMA LOCAL BASIN}

Moisture input is estimated from snowmelt and rainfall. Snowmelt depth estimate is based on snow survey and temperature forecast. Rainfall depth estimate is based on the local rain gage network. These depths are multiplied by the watershed area to compute volume. The area of Mehama Local Basin is 227 sq. miles. The SSARR Mehama Local Basin Model distributes each volume of moisture input among the baseflow, subsurface flow, and surface flow using three "splitting functions". Refer to Figure 2 for a flowchart of the computational structure of the SSARR model. 


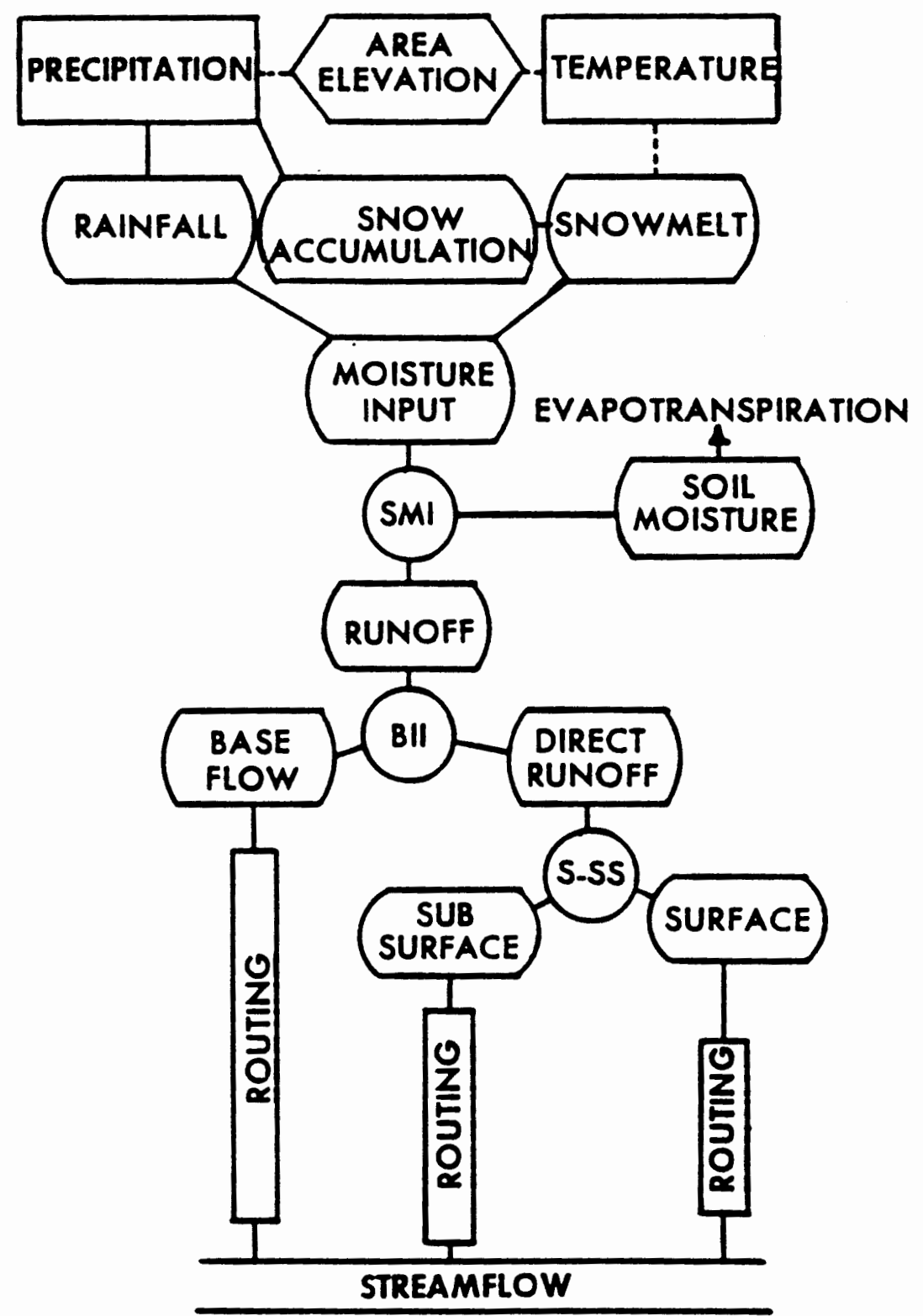

Figure 2. SSARR Model Schematic Flow-Chart. 
In the model, the processing of moisture input involves the following processes:

1. Separation of moisture input into either runoff or soil moisture, based on an index of soil moisture.

2. Calculation of an index of soil moisture.

3. Separation of runoff into direct runoff and baseflow as a function of an index of the baseflow infiltration index.

4. Separation of direct runoff into surface and subsurface flows.

The generated runoff for the period ( $t$ ) in inches, RGP, is the portion of moisture input that goes to runoff through baseflow, subsurface flow, and surface flow. The rest is stored in the hydrogeological structure of the watershed. This stored moisture is subject to loss by evapotranspiration. RGP is computed for each period based on this equation :

$\mathrm{RGP}=\mathrm{ROP} * \mathrm{WP}$

where ROP is the runoff percent. This is a function of SMI, soil moisture index; this function is conveyed by a table-look-up algorithm in the computer; and WP is the weighted net 
precipitation or distributed net precipitation, for the period, respectively, in inches. This is the net moisture input based on rainfall and/or snowmelt considerations.

The soil moisture index, SMI, is the amount of moisture stored in the hydrogeological structure of the watershed. SMI is updated at each time period by a difference equation which accounts for the input of moisture through precipitation and the output of moisture through runoff and evapotranspiration:

$\mathrm{SMI}_{\mathrm{i}}=\mathrm{SMI}_{\mathrm{i}-1}+(\mathrm{WP}-\mathrm{RGP})-(\mathrm{t} / 24) * \mathrm{KE} * \mathrm{ETI}$

where $S M I_{i}$ is the soil moisture index (in inches) at the end of period; SMI $\mathrm{I}_{-1}$ is the soil moisture index (in inches) at the beginning of period; WP is the weighted net precipitation or distributed net precipitation, for the period, due to rainfall and/or snowmelt; RGP is the generated runoff for the period, in inches; $t$ is the period length in hours; ETI is the evapotranspiration index, in inches per day, estimated from pan evaporation data and conveyed in the computer program as a monthly time series; and $\mathrm{KE}$ is a factor for reducing ETI on rainy days which is specified to the computer in a table of $\mathrm{KE}$ versus rate of precipitation in inches per day. 
The baseflow infiltration index, BII, controls the portion of runoff (RGP) that goes to baseflow. Like SMI, BII is also updated at each period based on a difference equation:

$$
\mathrm{BII}_{2}=\mathrm{BII}_{1}+\left(24 \times \mathrm{RG}-\mathrm{BII}_{1}\right)[\mathrm{t} /(\mathrm{TSBII}+0.5 \mathrm{t})]
$$

where BII2 is the Baseflow Infiltration Index (in inches per 24 hours) at beginning of period; BII 1 is the Baseflow Infiltration Index (in inches per 24 hours) at end of period; $t$ is the period length in hours; RG is the RGP/t which is runoff rate in inches per hour; and TSBII is the time delay or time of storage, typically ranges from 30 to 60 hours. This parameter represents the allowable rate of infiltration within the time period.

The baseflow percent (as a decimal), BFP, is the fraction of runoff going to baseflow and is a function of BII. It is computed based on a table-look-up algorithm. Thus, moisture input going to baseflow in inches, IMBF, is computed by this equation:

\section{$\mathrm{IMBF}=\mathrm{RGP} \times \mathrm{BFP}$}

The rest of the runoff goes to either surface and subsurface flow. Moisture input going to surface flow is IMS and to subsurface flow is IMSS. The sum of moisture input (in inches) going to surface and subsurface flow is RGS and is computed by this equation: 
RGS = RGP (1.0 - BFP)

PS is the fraction of RGS that goes into surface flow. The rest of RGS, namely (1-PS), goes to subsurface flow. PS is a function of RGS and is conveyed in the computer program as table-look-up algorithm. PS is a function of total input rate to surface and subsurface flow. This function was created by the River Forecast Center based on several premises :

1. Minimum surface runoff is $10 \%$ of total runoff rate (RGS).

2. Subsurface flow reaches maximum and remains constant when total runoff rate is above $200 \%$ of maximum input rate to the subsurface.

The moisture inputs to surface and subsurface flows are determined by the following equations:

$$
\begin{aligned}
& \mathrm{IMS}=\mathrm{RGP}(1.0-\mathrm{BFP}) \mathrm{PS} \\
& \mathrm{IMSS}=\mathrm{RGP}(1.0-\mathrm{BFP})(1-\mathrm{PS})
\end{aligned}
$$

For each time period, IMBF, IMS, and IMSS are computed and fed into the three routing algorithms, one for each flow components. The 
routing algorithms are based on the concept of a cascade of linear reservoirs.

\section{MODELING THE RUNOFF PROCESS OF MEHAMA LOCAL BASIN}

The cascaded linear reservoir algorithm is used in the SSARR to simulate the response of a watershed to moisture input. This algorithm assumes that water cascades down a series of reservoirs for which the outflow of each reservoir is linearly proportional to the storage in each reservoir. A single linear reservoir is described as :

$\mathrm{S}=\mathrm{T}_{\mathrm{S}} \mathrm{O}$

where $S$ is the storage (in cu. $\mathrm{ft}$.); $T_{S}$ is the time of storage, which is the proportionality factor between storage and outflow, unitless; and $O$ is the outflow (in cubic feet).

It is assumed that $T_{S}$ is constant. The linearity described in Eq 3.2 is a simplification from the observed rating curves of operating reservoirs. However, one must bear in mind that these cascaded "reservoirs" are only computational tools used for the conceptual representation of the flows in a watershed.

The flow of water through a reservoir is accounted using a continuity equation which says that the difference between mean inflow and mean outflow is equal to the change in storage. In discrete form, this can be written as: 
$0.5 \mathrm{t}\left[\mathrm{I}_{\mathrm{i}-1}+\mathrm{I}_{\mathrm{i}}\right]-0.5 \mathrm{t}\left[\mathrm{0}_{\mathrm{i}-1}+\mathrm{O}_{\mathrm{i}}\right]=\mathrm{S}_{\mathrm{i}}-\mathrm{S}_{\mathrm{i}-1}$

where $I i-1$ is the inflow at the beginning of the time period (cfs); $\mathrm{I}_{\mathrm{i}}$ is the inflow at the end of the time period (cfs); $\mathrm{O}_{\mathrm{i}-1}$ is the outflow at the beginning of the time period (cfs); $\mathrm{O}_{i}$ is the outflow at the end of the time period ( $\mathrm{cfs})$; $S_{i-1}$ is the storage at the beginning of the time period (cf); $S_{i}$ is the storage at the beginning of the time period (cf); and $t$ is the time duration or period length (in hours).

From Eq. 3.2, $\mathrm{T}_{\mathrm{S}}$ can be related to inflow and outflow as:

$$
T_{S}=\left(S_{i}-S_{i-1}\right) /\left(0_{i-1}+O_{i}\right)
$$

Defining, mean inflow, Im, as:

$$
\mathrm{m}_{\mathrm{i}}=(1 / 2)\left[\mathrm{I}_{\mathrm{i}-1}+\mathrm{I}_{\mathrm{i}}\right]
$$

$\mathrm{Eq} 3.3$, Eq 3.4, and $\mathrm{Eq} 3.5$ can be rewritten algebraically in the following working form :

$$
\mathrm{O}_{\mathrm{i}}=\mathrm{t}\left[\mathrm{Im}_{\mathrm{m}}-\mathrm{O}_{\mathrm{i}-1}\right] /\left[\mathrm{T}_{\mathrm{S}}+0.5 \mathrm{t}\right]+\mathrm{O}_{\mathrm{i}-1}
$$

To simplify, let 


$$
\mathrm{A}=\mathrm{t} /\left[\mathrm{T}_{\mathrm{S}}+0.5 \mathrm{t}\right]
$$

Now Eq 3.3 can be rewritten as:

$$
\mathrm{O}_{\mathbf{i}}=(1-\mathrm{A}) \mathrm{O}_{\mathbf{i}-1}+\mathrm{A} \mathrm{Im}_{\mathbf{i}}
$$

Eq 3.8 is the building block of each linear cascaded reservoir routing system used in modeling the flow of water in a watershed.

In a system of cascaded reservoirs, water flows in a cascaded manner from one reservoir to the next reservoir. Moisture input is the inflow to the top reservoir. Beginning at the second reservoir the inflow to each reservoir is the outflow from the preceding reservoir. An additional subscript is introduced to connote the position of the reservoir. Eq 3.8 will be used to derive the expression for each reservoir in a cascaded system. Hypothetically, a system of four reservoirs will be discussed.

Modifying Eq 3.8, water flow for the first reservoir is described by the following equation :

$$
\mathrm{O}_{1}=(1-\mathrm{A}) \mathrm{O}_{1_{\mathrm{i}-1}}+\mathrm{A} \mathrm{Im}_{\mathrm{i}}
$$

where

$\mathrm{O}_{1}$ is the outflow from the first reservoir at the beginning of the time period; $\mathrm{O}_{1-1}$ is the outflow from the first reservoir at the end of the time period; $\operatorname{Im}_{\mathrm{i}}$ is the mean moisture input; and $A$ is $t /\left[T_{S}+0.5 t\right]$; 
To develop the flow equation for the outflow from the second reservoir in the cascade, modify Eq 3.9 using Eq 3.5 so that

$$
\operatorname{Im}_{i}=0.5\left[\mathrm{I}_{\mathbf{i}-1}+\mathrm{I}_{\mathrm{i}}\right]
$$

Adding a subscript to the moisture input term to connote that it goes into the first reservoir, Eq. 3.9 becomes

$$
\mathrm{O}_{1 \mathrm{i}}=(1-\mathrm{A}) \mathrm{O}_{1_{\mathrm{i}-1}}+\mathrm{A} 0.5\left[\mathrm{I}_{1_{\mathrm{i}-1}}+\mathrm{I}_{1_{\mathrm{i}}}\right]
$$

where

$\mathrm{I}_{1 \mathrm{i}-1}$ is the moisture input going into the first reservoir at the beginning of the period; and $I_{1_{i}}$ is the moisture input going into the first reservoir at the end of the period.

Renaming the subscript in Eq 3.10, the outflow from the second reservoir is the following.

$$
\mathrm{O}_{2 \mathrm{i}}=(1-\mathrm{A}) \mathrm{O}_{2 \mathrm{i}-1}+(1 / 2) \mathrm{A} \quad\left[\mathrm{I}_{2 \mathrm{i}-1}+\mathrm{I}_{2 \mathrm{i}}\right]
$$

where

$\mathrm{O}_{2} \mathrm{i}$ is the outflow from the second reservoir at the beginning of the time period; $\mathrm{O}_{2 \mathrm{i}-1}$ is the outflow from the second reservoir at the end of the time period; $I_{2} i-1$ is the moisture input going into the second reservoir at the beginning of the 
period; and $\mathrm{I}_{2} \mathrm{i}$ is the moisture input going into the second reservoir at the end of the period.

By the nature of the cascade system, the outflow from the first reservoir is the inflow to the second reservoir. Thus,

$$
\begin{aligned}
& \mathrm{I}_{2 \mathrm{i}-1}=\mathrm{O}_{1_{\mathrm{i}-1}}, \text { and } \\
& \mathrm{I}_{2 \mathrm{i}}=\mathrm{O}_{1_{\mathrm{i}}}
\end{aligned}
$$

Eq 3.12 then becomes

$$
\mathrm{O}_{2 \mathrm{i}}=(1-\mathrm{A}) \mathrm{O}_{2 \mathrm{i}-1}+\mathrm{A} 0.5\left[\mathrm{O}_{1_{\mathrm{i}-1}}+\mathrm{O}_{1_{\mathrm{i}}}\right]
$$

Rearranging and substituting Eq 3.10, EQ 3.13 becomes

$$
\mathrm{O}_{2 \mathrm{i}}=(1-\mathrm{A}) \mathrm{O}_{2 \mathrm{i}-1}+(\mathrm{A} / 2)(2-\mathrm{A}) \mathrm{O}_{1_{\mathrm{i}-1}}+2(\mathrm{~A} / 2)^{\wedge} 2 \mathrm{Im}_{\mathrm{i}}
$$

Now, likewise, for the third reservoir,

$$
\begin{aligned}
\mathrm{O}_{3}= & (1-\mathrm{A}) \mathrm{O}_{3_{\mathrm{i}-1}}+(\mathrm{A} / 2)(2-\mathrm{A}) \mathrm{O}_{2} \mathrm{i}-1^{+}(\mathrm{A} / 2)^{\wedge} 2(2-\mathrm{A}) \mathrm{O}_{\mathrm{i}^{-} 1^{+}} \\
& 2(\mathrm{~A} / 2)^{\wedge} 3 \mathrm{Im}_{\mathrm{i}}
\end{aligned}
$$

And for the fourth reservoir,

$$
\mathrm{O}_{4 i}=(1-\mathrm{A}) \mathrm{O}_{4 \mathrm{i}-1}+(\mathrm{A} / 2)(2-\mathrm{A}) \mathrm{O}_{3_{\mathrm{i}-1}}+(\mathrm{A} / 2)^{\wedge} 2(2-\mathrm{A}) \mathrm{O}_{2 \mathrm{i}-1^{+}}
$$


$(\mathrm{A} / 2)^{\wedge} 3(2-\mathrm{A}) \mathrm{O}_{1_{\mathrm{i}-1}}+2(\mathrm{~A} / 2)^{\wedge} 4 \quad \mathrm{Im}_{\mathrm{i}}$

In the Mehama Local Basin Model, four reservoirs are used for modeling surface flow; three for subsurface flow; and three for baseflow.

For surface flow, Eq's 3.9, 3.13, 3.14, and 3.15 are used, substituting $A$ by $A_{s f}$ and $\operatorname{Im}_{i}$ by $\operatorname{Im}_{i_{s f}}$, where

$$
\begin{aligned}
\mathrm{A}_{\mathrm{sf}} & =\mathrm{t} /\left[\mathrm{Ts}_{\mathrm{sf}}+0.5 \mathrm{t}\right] \\
\mathrm{Im}_{\mathrm{i}_{\text {sf }}} & =\text { Mean moisture input for the ith period to surface flow } \\
& =(1 / 2)\left[\mathrm{I}_{\mathrm{i}-1_{\mathrm{sf}}}+\mathrm{I}_{\mathrm{i}_{\mathrm{Sf}}}\right]
\end{aligned}
$$

For subsurface flow, Eq's 3.9, 3.13 and 3.14 are used, substituting A by $A_{\text {ssf }}$ and $\operatorname{Im}_{i}$ by $\operatorname{Im}_{i_{s s f}}$, where

$$
\mathrm{A}_{\text {ssf }}=\mathrm{t} /\left[\mathrm{Ts}_{\mathrm{ssf}}+0.5 \mathrm{t}\right]
$$

$\mathrm{Im}_{\mathrm{i}_{\text {ssf }}}=$ Mean moisture input for the ith period to subsurface flow

$$
=(1 / 2)\left[\mathrm{I}_{\mathbf{i}-1_{\text {ssf }}}+\mathrm{I}_{\mathbf{i}_{\text {ssf }}}\right]
$$

For baseflow, Eq's 3.9, 3.13, and 3.14 are used, substituting $A$ by $A_{b f}$ and $\operatorname{Im}_{\mathrm{i}}$ by $\operatorname{Im}_{\mathrm{ibf}}$, where

$$
\begin{aligned}
\mathrm{A}_{\mathrm{bf}} & =\mathrm{t} /\left[\mathrm{Ts} \mathrm{sf}_{\mathrm{b}}+0.5 \mathrm{t}\right] \\
\mathrm{Im}_{\mathrm{ibf}_{\mathrm{bf}}} & =\text { Mean moisture input for the ith period to baseflow } \\
& =(1 / 2)\left[\mathrm{I}_{\mathrm{i}-1} \mathrm{bf}_{\mathrm{bf}}+\mathrm{I}_{\mathrm{ibf}}\right]
\end{aligned}
$$


The flow components used in modeling the Mehama Local Basin are surface flow, subsurface flow, and baseflow. The flow components differ from each other in their $T_{S}$ values. The surface flow has the smallest $T_{S}$ value because it generates runoff the most quickly in response to a moisture input. Likewise, the subsurface flow has larger $T_{S}$ value. Baseflow, with slowest response to moisture input, has the largest $T_{S}$ value.

SSARR is a real time model. As shown above, the hydrologic continuity of the represented watershed is represented by the soil moisture index, baseflow infiltration index, and the outflow (which is linearly proportional to storage) from each of the storages in the linear cascaded reservoir routing scheme for each of the three flow components. The indices are propagated to the next time step by a set of difference equations. 


\section{CHAPTER IV}

\section{TIME SERIES MODELING}

\section{INTRODUCTION}

For a real time model such as SSARR, the discrepancy between the model and the measurement is expressed in a time series of its residual. This time series could contain a pattern that is useful in making a better forecast. The purpose of time series modeling is to extract that pattern in the form of a time series model. The time series model could then be used in real time to augment the forecast made by SSARR and thereby generate more accurate forecasts. The background concepts of time series modeling pertinent to the creating such a time series model are presented in this chapter.

The discrete univariate linear time series models discussed herein are those with autoregressive and autoregressive moving average properties. Their basic properties and the algorithms to compute them are discussed. These concepts are found in many publications. The concepts and model estimation algorithm discussed in this chapter are derived and extracted principally from Salas, et al (1980) and Pandit and Wu (1983).

A time series is a periodically measured signal. Thus, whenever a system is measured at time intervals, a time series is formed (see Figure 3). Given enough resolution in the frequency of 
measurement, the recorded signal should, to some extent, reflect the behavior of the system. Analysis of this time series would result in a model form which simulates the behavior of the system.

Cantinuous Signal

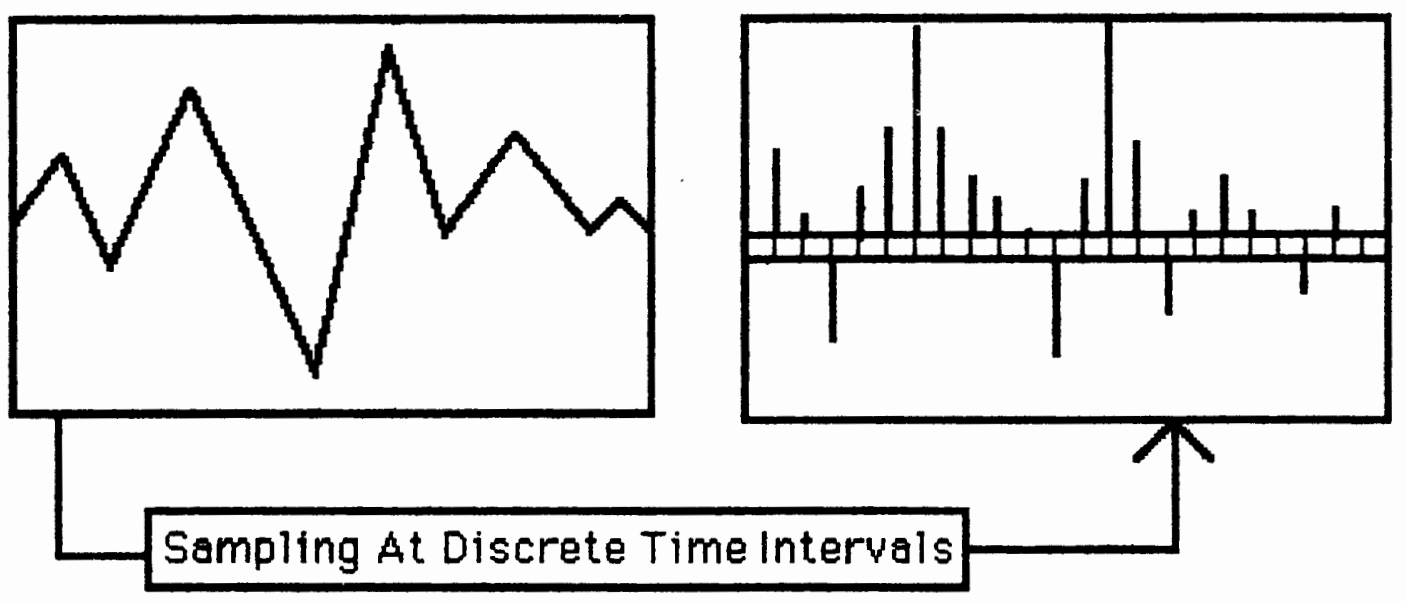

Figure 3. Discrete Time Series.

The physical mechanism of interest is called here the model variable $X$. The value of $X$ at a time step $t$ is denoted as $X_{t}$.

\section{WHITE NOISE}

Autoregressive and autoregressive-moving-average models are driven by white noise, et. A white noise is a time series with no time-dependent structure. Being completely unpredictable, there is no correlation whatsoever between the amplitude of a white noise at a given time and the amplitude of the white noise at any other time. This also implies that there is no trend or seasonality in a white noise. A trend is a systematic decrease or increase in the 
mean and/or variance while a seasonality is a pattern of cyclic fluctuation in the mean and/or the variance. Moreover, white noise exists only in discrete time. Theoretically, the formation of a white noise in continuous time requires infinite power.

By definition, the white noise has a zero mean, is normally distributed, and is uncorrelated in time; its variance characterizes power. A white noise is completely characterized by the following characteristics:

1. The auto-covariance function, $E\left[e_{t} e_{k+t}\right.$, is equal to zero except at $k=0$, where it equals to the variance of $e_{t}$.

2. A white noise is normally distributed with a zero mean and variance, $\mathrm{E}\left[\mathrm{e}_{\mathrm{t}} 2\right]$ of $\partial_{\mathrm{e}}$.

A time series is called stationary in the wide sense if it has constant zero mean with constant variance for all time. If the time series is also normally distributed, it is called stationary in the strict sense. Thus, a white noise is a series which is stationary in the strict sense.

A successful model must be able to generate a simulated time series that differs from the real time series only by a residual series that exhibits the behavior of a white noise series. Thus the adequacy of a model is checked by determining whether the residual has the characteristics of white noise. Using a long series, one can identify white noise by testing sample statistics and comparing 
them to the statistical characteristics of a theoretical white noise such as mean, skewness, and auto-covariance (or auto-correlation).

\section{PORTE MANTEAU TEST FOR WHITE NOISE}

Given an ARMA model, a less labor-intensive way to identify white noise is through Porte Manteau Test where the test statistic is:

$$
\mathrm{Q}=\mathrm{N} \sum \mathrm{ri}^{2}(\mathrm{e}) \text {; }
$$

where $r_{i}(e)$ is the correlogram at lag $i$ and the summation is taken from $\mathrm{i}=1$ to $\mathrm{L}$ where $\mathrm{L}$ is an integer equal to about 10 to $30 \%$ of the sample length.

$\mathrm{Q}$ is approximately chi-square - distributed at $\mathrm{H}$ degree of freedom, where $\mathrm{H}=\mathrm{L}-\mathrm{p}$, and $\mathrm{p}$ is the number of parameters in a given ARMA model. At a chosen significance level, when $Q$ is less than the chi-square critical value at $H$ degree of freedom, the residual series, e, is called a white noise.

\section{BASIC MODEL FORMS}

By definition, a time series model is assumed to fully capture the time-dependent structure in the time series the model represents. That is, the model-simulated time series and the actual time series should differ only by a white noise series.

An autoregressive model (AR) has a form such that the value of the model variable at any time step is a linear combination of the 
values of the model variable of previous time steps and a white noise component.

An $A R(N)$ model has the general form:

$X_{t}=a_{1} X_{t-1}+a_{2} X_{t-2}+a_{3} X_{t-3}+\ldots+a_{N} X_{t-N}+e_{t}$

where $a_{i}$ is a weighting coefficient for the $i^{\text {th }}$ previous model variable, $\mathrm{N}$ is any natural number $(1,2,3$, etc. $)$, and $e_{t}$ is the white noise input of the present time step.

An autoregressive-moving-average (ARMA) model has a form such that the value of the model variable at any time step is a linear combination of the values of the model variable of previous time steps and the white noise terms of previous time steps.

An $\operatorname{ARMA}(\mathrm{N}, \mathrm{M})$ model has the general form :

$$
\begin{aligned}
X_{t}=a_{1} X_{t-1}+a_{2} X_{t-2}+ & a 3 X_{t-3}+\ldots+a N X_{t-N}-m_{1} e_{t-1}- \\
& m 2 e_{t-2}-m_{3} e_{t-3}-\ldots-m M e_{t-M}+e_{t}
\end{aligned}
$$

where $m_{i}$ is the weighting coefficient for the $i^{\text {th }}$ previous white noise and $N$ and $M$ are any natural numbers $(1,2,3$, etc. $)$. 
An AR model is a special form of ARMA model, namely an $A R(N)$ is an $\operatorname{ARMA}(\mathrm{N}, 0)$. Therefore, the ARMA form, Eq 4.1, is referred to as the standard model, with these designs :

1. The subscripts denote time steps.

2. The a's and m's are model parameters, which define the nature of a particular model and must be computed when modeling a time series.

3. $\mathrm{N}$ is the number of the autoregressive terms.

4. $\quad \mathrm{M}$ is the number of the moving average terms.

5. $\mathrm{X}$ is the univariate model variable which is a measured attribute of a real world system.

6. The e's are the model residual which is assumed to be a white noise.

To model a particular time series, one needs to find the ARMA model form by estimating the integer value of $N$ and $M$ and the values of the model parameters a's and m's.

White noise is the input to every AR and ARMA time series model. Interestingly, white noise is the "purpose" of time series 
modeling. Rearranging the general form of an ARMA model, EQ 4.1, we have:

$$
\begin{array}{r}
e_{t}=X_{t}-\left\{a_{1} X_{t-1}+a_{2} X_{t-2}+a_{3} X_{t-3}+\ldots+a N X_{t-N}-m_{1} e_{t-1}-\right. \\
\left.m_{2} e_{t-2}-m_{3} e_{t-3}-\ldots .-m_{M} e_{t-M}\right\}
\end{array}
$$

This equation shows that to model is to reduce a time series to white noise. In other words, an adequate model must have diagnosable white noise residual.

\section{BASIC MATHEMATICAL CHARACTERISTICS}

\section{OF A TIME SERIES MODEL}

The mathematical characteristics of an ARMA model hinge on its relations to the values of the model variable $(\mathrm{X})$ and white noise terms (e) of previous time steps; these relations are expressed by the Green's Functions, Inverse Functions, and Auto-Covariance Functions. Pandit and Wu (1983) showed that the ARMA model can be written as

$P(B) X t=Q(B) e_{t}$

where $\mathrm{P}(\mathrm{B})$ is a $\mathrm{N}$ degree polynomial; $\mathrm{Q}(\mathrm{B})$ is a $\mathrm{M}$ degree polynomial; and B is the backward shift operator.

Rearranging, we can arrive at two equations for the dual transformations between $X_{t}$ and $e_{t}$ : 


$$
X_{t}=\{Q(B) / P(B)\} \text { et }
$$

and

$$
e_{t}=\{P(B) / Q(B)\} X_{t}
$$

The term $\{Q(B) / P(B)\}$ gives rise to Green's Functions; the term $\{P(B) / Q(B)\}$ gives rise to the inverse functions; and, additionally, the statistical correlations among the model variable $X$ of various time steps are described by the auto-covariance functions.

\section{GREEN'S FUNCTIONS}

The Green's functions govern the dependence of present model variable $X_{t}$ on the previous white noise inputs, essentially showing how every ARMA model can be represented by an equivalent $\mathrm{MA}(\infty)$ model. By long hand division of Eq 4.2 and rearranging,

$$
X_{t}=e_{t}+G_{1} e_{t-1}+G_{2} e_{t-2}+G_{3} e_{t-3}+G_{4} e_{t-4}+\ldots \ldots .
$$

or, using the backward shift operator,

$$
X_{t}=\left(G_{0} B^{0}+G_{1} B^{1}+G_{2} B^{2}+G_{3} B^{3}+G_{4} B^{4}+\ldots\right) e_{t}
$$

Thus, the Green's function can be written as 
$\{\mathrm{Q}(\mathrm{B}) / \mathrm{P}(\mathrm{B})\}=\sum \mathrm{G}_{\mathrm{j}} \mathrm{Bj}$

where the $G$ 's are the Green's Functions and the summation is taken $\mathrm{j}=0$ to $\infty$;

The value of $G_{j}$ indicates how well the input at the $j^{\text {th }}$ time step is remembered by the present model variable. If a $G_{j}$ is very large, the input to system at the $\mathrm{j}^{\text {th }}$ time step will be clearly remembered for more time steps, and, moreover, it will take the model a longer time to return to its equilibrium value.

Based on long hand division, Green's functions have the following characteristics:

$$
\begin{aligned}
& G_{j}=g_{1} u_{1}^{j}+g_{2} u_{2}^{j}+g_{3} u_{3}^{j}+\cdots+g_{N} u_{N}^{j} \\
& \text { where } u_{1}, u_{2}, u_{3}, \ldots, u_{N} \text { are roots of } \\
& u^{N}-a_{1} u^{N-1}-a_{2} u^{N-2}-a_{3} u^{N-3}-\cdots-a_{N}=0 \text {; }
\end{aligned}
$$

The a's are model parameters of the autoregressive terms of the $\operatorname{ARMA}(\mathrm{N}, \mathrm{N}-1)$ model. The g's are functions of u's, m's, and $\mathrm{N}$.

The asymptotic stationarity condition ensures that the value of $\mathrm{X}_{\mathrm{t}}$ will not increase without bound. This condition requires that the absolute value of $u_{i}$ must be smaller than 1 . For any time series model meeting the asymptotic stationarity condition, if no more input is injected, $\mathrm{X}$ will asymptotically return to its equilibrium value given enough time. In other words, for a stationary time series 
model, the memory of previous inputs is gradually lost. When stationarity conditions are met, the equivalent $\mathrm{MA}(\infty)$ model can be easily approximated by an $\mathbf{M A}(\mathbf{M})$ where $M$ is some finite number. Since $u_{i}$ is affected only by the AR terms of the model, one can conclude that, given an ARMA(N,M) model, stationarity conditions of the model are based on only the values of the AR-parameters.

Finally, when a stochastic trend is present in the data series, some $u_{i}$ 's have absolute values very near to or equal to 1 . Stochastic seasonality is present when some of the roots, $u_{i}$ 's, are imaginary and their absolute values close to 1 . Periods of such seasonal series can be determined from the values of the conjugate roots.

\section{INVERSE FUNCTIONS}

Inverse functions govern the dependence of the present model noise $e_{t}$ on previous values of the model variable. It shows that every ARMA can be equivalently represented by an $\operatorname{AR}(\infty)$.

Inverse functions and Green's function are dual characteristics of a ARMA model. Therefore the derivation of the inverse functions is directly analogous to that of Green's functions.

By long hand division of $\mathrm{Eq} \mathrm{4.3,} \mathrm{and} \mathrm{again} \mathrm{using} \mathrm{the} \mathrm{backward}$ shift operator, the inverse function is

$$
\{\mathrm{P}(\mathrm{B}) / \mathrm{Q}(\mathrm{B})\}=-\sum \mathrm{Ij}_{\mathrm{j}} \mathrm{j}
$$

where I's are the Inverse functions; summation taken from $\mathrm{j}=0$ to $\infty$; and $I_{0}$ is -1 by definition. 
Going back to the analysis of Green's functions in previous subsection and replacing all the a's (AR-parameters) by m's (MAparameters), u's by v's, and $N$ (the order of $A R$ sub-system) by $M$ (the order of MA sub-system), the following equation sets can be obtained for characterizing the inverse functions:

In general, for an ARMA(N,M) model,

$$
\begin{gathered}
l_{j}=n_{1} v_{1}^{j}+n_{2} v_{2}^{j}+n_{3} v_{3}^{j}+\cdots+n_{M} v_{M}^{j} \\
\text { where } v_{1}, v_{2}, v_{3}, \ldots, v_{M} \text { are roots of } \\
v^{M}-m_{1} v^{M-1}-m_{2} v^{M-2}-m_{3} v^{M-3}-\cdots-m_{M}=0 ;
\end{gathered}
$$

Analogous to the stationarity conditions related to the ARparameters within an ARMA(N,M) model, there are invertibility conditions related to the MA-parameters. Invertibility conditions are met when all the absolute values of all the roots, $\mathrm{vi}_{\mathrm{i}} \mathrm{s}$, are smaller than 1 .

When invertibility conditions are met, the present model residual's memory of previous $X$ values will gradually fade. The value of residual is thus bounded and the equivalent $\operatorname{AR}(\infty)$ model of the ARMA model can be approximated by an $\operatorname{AR}(M)$ where $M$ is some finite number. This forms the basis for estimating model parameters by mathematical inference, discussed in later section. 
When invertibility conditions are violated, the more distant in time an $X$ value is into the past, the stronger its influence will be on the present $e_{t}$. This behavior is physically unrealistic. To achieve a physically meaningful model and ensure that the model software will run properly, invertibility condition must be scrupulously imposed on the MA parameters of every ARMA model.

A remedy does exist when the estimated ARMA(N,M) model turns out to be non-invertible. For an ARMA model, when roots vi's with absolute values larger than 1 are found, they are replaced by their reciprocals. The MA parameters are then recomputed using the second equation. The new MA parameters will give rise to new AR parameters. If the direct-least-square search method is used, problems with invertibility should not arise, due to the least square criterion.

On the other hand, while invertibility condition must always be imposed, stationarity conditions must always be preserved because they are basic model characteristics. It is therefore more scientific not to pre-process data series and remove apparent patterns. The roots of AR parameters will show whether the series is stationary or non-stationary. In the non-stationary cases, the roots will quantitatively indicate seasonality and/or trend.

When the stationarity and invertibility conditions are fulfilled, there will be a finite output $X_{t}$ for every finite input $e_{t}$, as described by $\mathrm{Eq} 4.2$ and $\mathrm{Eq}$ 4.3. In practice, when estimating an ARMA model, parameters are first estimated; then invertibility and 
stationarity conditions are tested on the roots of MA and AR parameters.

\section{AUTO-COVARIANCE FUNCTIONS}

The auto-covariance functions describe the statistical dependence among the values of model variable at different time steps. By definition :

$\operatorname{acf}(k)=E\left[X_{t} X_{t-k}\right]$

where $\operatorname{acf}(k)$ is the autocovariance function of the $X_{t}$ process at time lag $\mathrm{k}$.

It can be shown that the theoretical auto-covariance (acf) of a $\operatorname{ARMA}(\mathrm{N}, \mathrm{M})$ model has the following characteristics:

$\operatorname{acf}(k)=d_{1} u_{1} k+d_{2} u_{2} k+d 3 u_{3}^{k}+\ldots+d N u N^{k}$

where $\mathrm{k}$ is time lag; $\left\{\mathrm{u}_{1} \mathrm{k}, \quad \mathrm{u}_{2}^{\mathrm{k}}, \mathrm{u}_{3} \mathrm{k}, \ldots, \mathrm{uN}^{\mathrm{k}}\right\}$ are the roots $u_{i}$ raised to the kth power; and $\left\{d_{1}, d_{2}, d_{3}, \ldots, d_{N}\right\}$ are some functions of the variance of the white noise, the moving average parameters of the model (m's), and the u's and g's from the discussion of Green's Functions. 
Given a time series, the value of $\operatorname{acf}(k)$ can be estimated by :

$$
\operatorname{acf}(k)=(1 / N) \sum\left(X_{t}-\text { mean }\right)\left(X_{t-k}-\text { mean }\right)
$$

where $, \mathrm{k}+1<\mathrm{t}<\mathrm{N}, \mathrm{N}$ is the number of data points in the time series, and the mean is estimated by sample mean which is $\left(\Sigma \mathrm{X}_{\mathrm{t}}\right) / \mathrm{N}$ for all $\mathrm{t}$.

Unlike Green's and inverse functions, auto-covariance is estimated directly from a given sample time series. Given an ARMA model, the theoretical auto-covariance function is computed from the model form. When the model completely represents the time series data, the two auto-covariance functions become equivalent.

The theoretical auto-covariance function is directly related to Green's functions and the variance of the white noise:

Consider this aforementioned equation:

$$
\begin{gathered}
x_{t}=\sum_{j=0}^{\infty} G_{j} e_{t-j} \\
\left(G_{0}=1\right)
\end{gathered}
$$

It is known that

$$
E\left[e_{t} e_{t-k}\right]=\left\{\begin{array}{l}
0 \text { if } k \neq 0 \\
1 \text { if } k=0
\end{array}\right\}=\partial \text { variance(e) }
$$

$\partial$ is Kronecker delta. 
The acf is related to the Green's Functions, as follows :

Deriving,

$$
\begin{aligned}
\operatorname{acf}(k) & =E\left[X_{t} X_{t-k}\right] \\
& =E\left(\sum_{j=0}^{\infty} G_{j} e_{t-j}\right)\left(\sum_{j=0}^{\infty} G_{j} e_{t-(k+j)}\right) \\
& =\sum_{i=0}^{\infty} \sum_{j=0}^{\infty} G_{i} G_{j} \partial_{i-(k+j)} \text {.variance(e) } \\
& =\text { variance(e) } \sum_{j=0}^{\infty} G_{j} G_{j+k}
\end{aligned}
$$

Hence, given an ARMA model form, the $\operatorname{acf}(k)$ can be calculated from the Green's Functions.

The theoretical auto covariance functions and sample autocovariance functions can be conveniently compared visually when they are plotted against time lag k. After an $\operatorname{ARMA}(\mathrm{N}, \mathrm{M})$ model is fitted to a time series data, one can compute the sample autocovariance at different lags from the data and the theoretical autocovariance from the model's Green's functions at different lags. If the corresponding values and patterns of the 2 groups are similar, one may conclude that the fitted model is in agreement with the behavior of the data. 


\section{MODEL ESTIMATION TECHNIQUES}

This section deals with the pragmatic issues of creating a time series model from a time series data set. Model estimation involves first the selection of a set of candidate models and then the estimation of the best model from among the candidates.

\section{Normalization of Time Series Data}

Before any modeling analysis is performed, every time series, $X_{t}$, should be first normalized about its sample mean and sample standard deviation by the following transformation:

$$
\mathrm{Z}_{\mathrm{t}}=\frac{\underline{X}_{\mathrm{t}}-\text { (sample mean) }}{\text { (sample st. dev.) }}
$$

for all time steps.

The transformed time series, $Z_{t}$, thus has zero mean, and a variance of 1 . While it may resemble a normal distribution, the skewness of the original time series is preserved.

Estimating An AR Model By Multivariate Linear Least Square Regression

Consider a univariate regressive system:

$$
Y_{t}=a_{1} X_{t}+e_{t}
$$


where $Y_{t}$ is the dependent variable; $X_{t}$ is the independent variable; and $e_{t}$ is a white noise sequence.

One can show that the sum of squares of residual, $e_{t}$, is at its minimum when

$$
\mathrm{a}_{1}=\sum \mathrm{Y}_{\mathrm{t}} \mathrm{X}_{\mathrm{t}} / \sum \mathrm{X}_{\mathrm{t}}{ }^{2}
$$

where the summation is over all $\mathrm{t}$.

Generalizing to the multivariate case, $Y_{t}$ is a linear combination of a finite number of variables, $X_{1 t}, X_{2 t}, \ldots, X_{k t}$; the model is:

$$
Y_{t}=a_{1} X_{1 t}+a_{2} X_{2 t}+a_{3} X_{3 t}+\ldots+a_{k} X_{k t}+e_{t}
$$

Rewriting in matrix form,

$$
Y_{t}=\mathbf{A ~ X} \mathbf{X}_{t}+e_{t}
$$

where $X_{t}$ is $\left[\begin{array}{lllllll}X_{1} t & X_{2 t} & X_{3} t & X_{4} & \ldots & X_{k t}\end{array}\right]^{T}$; $A$ is $\begin{array}{lllll}a_{1} & \text { a2 } & \text { a3 } & \ldots\end{array}$ ak $] ;$ and $e_{t}=\left[\begin{array}{llllll}e 1 t & e 2 t & e 3 t & e 4 t & \ldots & e k t\end{array}\right]^{T}$.

Given a data set of $\mathrm{N}$ points, to estimate a model in the form of Eq. 4.6, one needs to compute the values of the elements (a's) in 
matrix A that minimize the square error function. The result of this minimization is:

$$
\mathbf{A}=\left[\mathbf{X}^{\mathrm{T}} \mathbf{X}\right]^{-1} \mathbf{X}^{\mathrm{T}} \mathbf{Y},
$$

$$
\begin{aligned}
& \text { where } \quad x=\left[\begin{array}{ccccc}
x_{11} & x_{21} & x_{31} & \ldots & x_{k 1} \\
x_{12} & x_{22} & x_{32} & \ldots & x_{k 2} \\
x_{13} & x_{23} & x_{33} & \ldots & x_{k 3} \\
\vdots & \vdots & \vdots & \vdots & \vdots \\
x_{1 N} & x_{2 N} & x_{3 N} & \ldots & x_{k N}
\end{array}\right] \text {, and } \\
& \mathrm{Y}^{\mathrm{T}}=\left[\begin{array}{llllll}
\mathrm{Y}_{1} & \mathrm{Y}_{2} & \mathrm{Y}_{3} & \ldots & \ldots & . . \\
\mathrm{Y}_{\mathrm{N}}
\end{array}\right], \quad \text { for a data set of } \mathrm{N} \text { points. }
\end{aligned}
$$

Now consider the general form of an $\operatorname{AR}(k)$ model :

$$
X_{t}=a_{1} X_{t-1}+a_{2} X_{t-2}+a_{3} X_{t-3}+\ldots+a_{k} X_{t-k}+e_{t}
$$

The form is the same as a general regression model, Eq 4.5. By observation and equating the analogous terms, one arrives at the following general formulations for computing the parameter of the AR(k) model:

Given a time series, $\left\{\mathrm{X}_{1}, \mathrm{X}_{2}, \mathrm{X}_{3}, \ldots \ldots ., \mathrm{X}_{\mathrm{N}}\right\}$, 


$$
\begin{aligned}
\mathbf{A} & =\left[\begin{array}{lllll}
\mathbf{a}_{1} & \mathbf{a} 2 & \mathbf{a 3} & \ldots & \mathbf{a k}
\end{array}\right] \\
& =\left[\mathbf{X}^{\mathbf{T}} \mathbf{X}\right]^{-1} \mathbf{X}^{\mathbf{T}} \mathbf{Y}
\end{aligned}
$$

where

$$
\begin{aligned}
& \mathrm{Y}^{\mathrm{T}}=\left[\begin{array}{llllll}
\mathrm{X}_{\mathrm{k}+1} & \mathrm{X}_{\mathrm{k}+2} & \mathrm{X}_{\mathrm{K}+3} & \ldots & \ldots & . . \\
\mathrm{X}_{N}
\end{array}\right] \text {, and }
\end{aligned}
$$

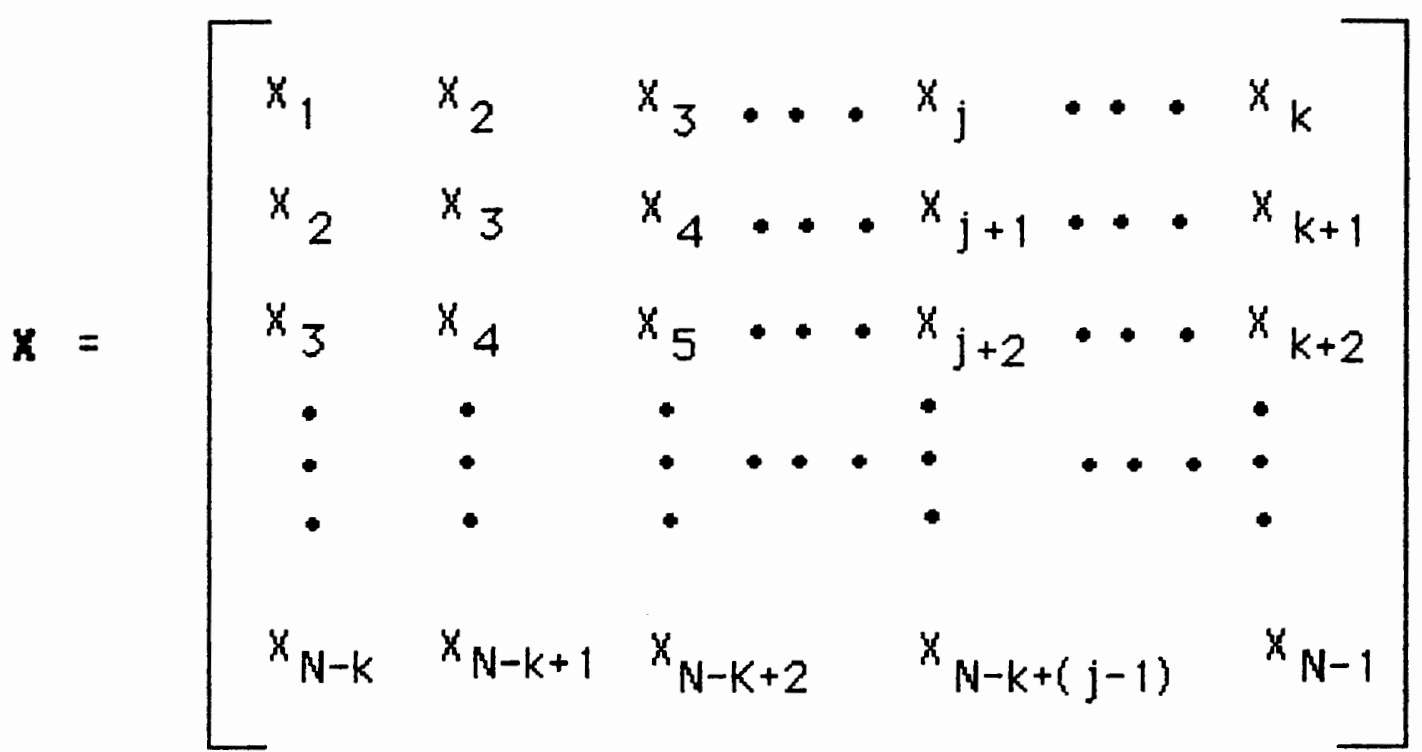

Note that the computations required in $\mathrm{Eq} 4.6$ are readily achievable with a digital computer. Once the time series data are arranged in the form of $\mathbf{X}, \mathbf{A}$ can be computed by a computer programs that manipulates matrices and vectors. The stationarity of each $\operatorname{AR}(\mathrm{N})$ model should be checked by solving for its roots, as described earlier. Models that do not meet stationarity condition should not be used due to the lack of physical meaning and the potential value overflow during computation. 
Estimating An ARMA Model By Mathematical Inference

An ARMA model can be approximated by an equivalent AR model of infinite order. However, practically speaking, because of the invertibility conditions imposed on the ARMA model, the inverse functions, I's, of high orders are small. Thus, the equivalent $\operatorname{AR}(\infty)$ model can be approximated by some $\operatorname{AR}(N)$ model where $N$ is an adequately large but finite number. As a rule of thumb, for estimating $\operatorname{ARMA}(n, k)$ based on an $\operatorname{AR}(N), N=\max (n, k)+k$. Pandit and Wu (1983) provide a step by step description of how the estimation can be accomplished. When the number of data points in a time series is large, this approach is most effective and further estimation using more exact computational procedures becomes optional.

\section{Least Square Direct Search in Multi-Dimensions}

Given an assumed model form and a data series, an alternative to the mathematical inference approach is a technique which computes model parameters by iterative direct search with a digital computer. The search criteria is the minimum sum of squared error. The goal is to find the values of the model parameters that will minimize the model residual sum of square function by adjusting the parameters within the range of values confined by stationarity and invertibility conditions.

To fit an $\operatorname{ARMA}(1,1)$ model to a time series, $X_{t}$, the goal is to find $\mathrm{a}_{1}$ and $\mathrm{m}_{1}$ that will minimize the sum of residual square, 
$\sum e^{2}{ }^{2}$, for all $t$ where $e_{t}=X_{t}-a_{1} X_{t-1}+m_{1} e_{t-1}$ by adjusting a1 and $\mathrm{m} 1$ within their ranges of values allowed by invertibility and stationarity conditions. These conditions are:

$$
\begin{aligned}
& -1<=\mathrm{u}_{1}<=+1 \quad \text {; and } \\
& -1<\mathrm{v} 1<+1
\end{aligned}
$$

where u's and v's are related by these two equations:

$$
\begin{aligned}
& \mathrm{u}_{1}-\mathrm{a}_{1}=0 \\
& \mathrm{v}_{1}-\mathrm{m}_{1}=0
\end{aligned}
$$

Given an assumed model, this method should succeed in finding the best fit model in the least squares sense. Moreover, goodness of fit of the model is indicated by its associated value of sum of squared residual, for comparison between candidate models.

Sequential Algorithm for Estimating the Optimal Time Series Model

So far, this discussion has dealt with the estimating the parameters of an AR or ARMA model given the model form. This section deals with the ultimate question of estimating the model form that will best fit a given time series. This calls for a sequential algorithm by which a number of candidate models are estimated and the best model is selected. This algorithm (Pandit 
and $\mathrm{Wu}, 1983)$ is based on computing $\mathrm{AR}$ models by regression and inferring ARMA models from AR models. Systematically, several (about 8 to a dozen) low order AR and ARMA models are produced, and from them the most adequate model is selected.

The algorithm proceeds as follows:

Step 1. Create $\operatorname{AR}(\mathrm{k})$ models for $\mathrm{k}=1,2,3$, and 4 .

Step 2. By inference, estimate $\operatorname{ARMA}(1,1), \operatorname{ARMA}(2,1)$, $\operatorname{ARMA}(3,1)$, and $\operatorname{ARMA}(2,2)$ using the $\operatorname{AR}$ models estimated at step 1. If the reduction in sum of residual square of an inferred ARMA model is very small when compared to the corresponding $A R$ model, the modeling process is assumed to have reached the "point of best fit" in the sense that a more complex model would not bring about significant improvement.

Step 3. Eliminate candidate models that do not produce a white noise model residual based on the Porte Manteau lack of fit test.

Step 4. Compare the performances of all candidate models and choose the best model, based on the following three performance criteria: 
a. Compare their sums of squares of the residuals and number of model parameters using the F-test, using the following test statistics:

$$
F=\left[\left(A_{1}-A_{0}\right) / 4\right] /\left[A_{0} /(N-4 n-4)\right]=F(4, N-4 n-4)
$$

where $A_{0}$ is the sum of squares of residuals for the $\operatorname{ARMA}(2 n+2,2 n+1)$ model; $A_{1}$ is the sum of squares of residuals for $\operatorname{ARMA}(2 n, 2 n-1)$, the model from last step; and $\mathrm{N}$ is the number of data in the time series. The value of $F$ indicates whether the reduction in sum of square of residuals going from $\operatorname{ARMA}(2 n, 2 n-1)$ to $\operatorname{ARMA}(2 n+2,2 n+1)$ is significant or not. When $\mathrm{F}$ is less than the tabulated value (say, at $5 \%)$, it indicates that $\operatorname{ARMA}(2 n, 2 n-1)$ is adequate. Sequential fitting is then stopped and the neighborhood check should begin. When used properly, A0 is always smaller than A1.

b. Compare the whiteness of the residual series using the mean, skewness, and auto-covariance.

c. Compare their measures of model parsimony using the Akaike Information Criterion (AIC) and choose the model with the smallest AIC. Parsimony of 
model form means minimum number of model variables required for an adequate representation of the system. In other words, a parsimonious model is one that can represent adequately the dynamics of the real system with minimum amount of model structures. The Akaike Information Criterion is a convenient measure of parsimony:

For an $\operatorname{ARMA}(p, q)$ model,
$\operatorname{AIC}(p, q)=N \ln ($ sum of squares of noise $/ N)+2(p+q)$
where $\mathrm{N}$ is the number of data point.

This algorithm can be modified for estimating models of higher orders. However, for the purpose of this study, it has proven sufficient. 


\section{CHAPTER V}

\section{STATE SPACE MODELING}

The derivation of the Kalman filter in modern estimation theory utilizes the state space form for system representation. Hence, in order to implement the filter algorithm, the SSARR Mehama Local Basin Model must first be re-written in state space.

\section{BASIC NOTIONS}

In general, state is defined as "a set of circumstances or attributes characterizing a person or thing at a given time". From the standpoint of mathematical modeling, for a dynamic system, the states at a given time, together with the input(s) at subsequent times, uniquely determine the states at subsequent times. The concepts discussed in this chapter are also discussed in many references, such as Szollosi-Nagy (1976), Gelb (1974), and Maybeck (1979).

Considering a discrete dynamic system, for any time step, the dynamic characteristics of the system are completely conveyed by the states. The system is driven forward (or backward) by a given input one time step at a time. Knowing the states and input of a time step, the states of the next time step can by uniquely computed. 
States change in value through time based on the dynamic structure of the system and in response to inputs to the system. The dynamic structure of the system dictates how the states of a time step are related to the states of the next time step. To go from one time step to the next, the state is processed by the transition relation and then the input is added to some or all states depending on the nature of the system.

The states of a dynamic system are ever-changing, either in response to the an input or "relaxing" toward their equilibrium values when input is absent. The states can be brought exponentially closer to equilibrium by propagating the system with zero input through many time steps. Near equilibrium, the effect of all nonzero input are almost undetectable in the values of the states.

For example, consider a simple deterministic auto-regressive model representing the streamflow of a hypothetical reach of river:

$$
\begin{aligned}
& X_{t}=0.95 X_{t-1}+C_{t-1} \\
& Y_{t}=X_{t}
\end{aligned}
$$

where $X_{t}$ is the streamflow at time $t$ (in CFS); $X_{t-1}$ is the streamflow at time $\mathrm{t}-1$ (in CFS); $\mathrm{C}_{\mathrm{t}-1}$ is the moisture input to the river reach at time $t-1$ (in CFS); and $Y_{t}$ is the observation of streamflow at time $t$. 
Eq 6.1 says that, in the absence of moisture input, the streamflow on a given day is $95 \%$ of that on the previous day. Moreover, there is no discrepancy between the measurement and model.

The state of this streamflow system is $X$, the streamflow. The dynamic behavior of the river at time step $t$ is fully characterized by $X_{t}$. Knowing the streamflow and moisture input of a time step, the streamflow of the next time step can be uniquely computed. The dynamic structure of the system is conveyed by the coefficient 0.95 which dictates the transition of the state through time. In the absence of non-zero input, the state "relaxes" exponentially toward its equilibrium value which is zero CFS.

\section{STATE SPACE MODEL}

The state space of a system is the set of possible values of the states. State space modeling is a modeling format in which a multivariate dynamic model is written in the following two state equations.

$$
\begin{aligned}
& \mathbf{X}_{t+1}=T_{t+1, t} \mathbf{X}_{t}+F_{\mathbf{t}_{t}} \\
& \mathbf{Z}_{t+1}=\mathbf{H} \mathbf{X}_{\mathrm{t}+1}
\end{aligned}
$$

where $\mathbf{X}_{\mathbf{t}+1}$ is the state vector containing the states of the model of time step $t+1 ; X_{t}$ is the state vector containing the states of the model of time step $t ; T$ is the state transition 
matrix, converting states from time $t$ to time $t+1$; $u_{t}$ is the input vector; $Z_{t+1}$ is the observation vector of time step $t+1$; $\mathbf{H}$ is the observation matrix; and $\mathbf{F}$ is the input matrix, which preprocesses the input vector and affects the way the input vector affects the states

The first equation expresses in the form of a difference equation the current state vector as a function of the previous state vector and the current system input vector. The second expresses the current system measurement (or output) vector as a function of the current state vector. While the system input and system measurement (or output) always have a physical meaning and real world counterparts, the states of the system model may be solely a mathematical convenience that define the causalities of the model internal structure and do not always all have real world counterparts. A mathematical model could have more than one state space representation.

The state equations for a given mathematical model can be achieved by observation. For example, consider the simple streamflow model of Eq 6.1. No vector or matrix is needed for Eq 6.1 because the system is univariate. However, one can rewrite this system with vectors and matrices of dimension one. Eq. 6.1 becomes the following :

$$
\mathbf{X}_{\mathrm{t}}=[0.95] \mathbf{X}_{\mathrm{t}-1}+[1] \mathrm{C}_{\mathrm{t}-1}
$$




$$
\mathbf{Z}_{\mathrm{t}}=[1] \mathbf{X}_{\mathbf{t}}
$$

Note that $T$ the transition matrix, is an $1 \times 1$ matrix [0.95] and $H$ the observation matrix is an $1 \times 1$ matrix [1]. The state vector is simply the streamflow $X$, now a one dimensional vector $X$. Likewise moisture input and observation are one dimensional vectors $\mathbf{C}$. and $\mathbf{Z}$. Input matrix, $\mathbf{F}$, is now an $1 \times 1$ matrix [1].

The dynamic characteristics of the a state space model are conveyed completely by the state transition matrix, $T$. The scheme of observing the modeled system is conveyed completely by the observation matrix, H. Although the one dimensional state space system of Eq 6.1 may seem trivial, at higher dimension, such as the case with Mehama Local Basin Model, the formulation of $\mathbf{X}, \mathbf{T}$, and $\mathbf{H}$ is not trivial.

\section{SELECTING MODEL STATES}

Given an existing mathematical model, the states of the system can be identified following the definition that the states must convey all dynamic (time-dependent) information of a system at a time. That is, all attributes in the model (or, interchangeably, system) that are necessary in describing the dynamic characteristics of the model at a given time should be selected as states. To ensure that the resulting model is of minimum vector dimension, the selection of states must not be redundant.

When selecting states for a complex system, one might also consider the nature of measurement and model update. Namely, the 
model variables that need to be updated by measurement and the model variables that are measured, directly or indirectly should be included as states of the system.

The measurement, $Z_{t}$, is crucial in updating the states so that the model will represent the latest measured conditions of the real system. Successful model update is dependent on the information derived from the measurement. When the values of the model states can be uniquely determined for a certain time step based on the information provided by the measurements and the inputs of a finite number of time steps, the model is said to be observable. In designing a state space model, one should make every effort to achieve an observable model.

The concept of observability (Gelb, 1984) is presented mathematically in the following. Assume a state space model with an n-dimensional state vector and scalar measurement:

$$
\begin{aligned}
& \mathbf{X}_{t+1}=\mathbf{T} \mathbf{X}_{t}+\mathbf{u}_{t} \\
& Z_{t+1}=\mathbf{H} \mathbf{X}_{t+1}
\end{aligned}
$$

where $\mathbf{T}=\mathbf{T}_{\mathrm{t}+1, \mathrm{t}}$ is the transition matrix converting the states from time $t$ to time $t+1$.

Note that in this case, $Z_{t+1}$, the measurement is a scalar, not a vector. This situation of a scalar measurement is analogous to the Mehama Local Basin Model in which the streamflow (in scalar form) 
is measured, despite the vector (or multivariate) nature of the states of the model as discussed in later sections. Since measurement is a scalar and state vector is of dimension $n, H$ is a $n$ rows by 1 column matrix.

To uniquely determine states at time step $0, \mathrm{X}_{0}$, given the inputs and measurements of a number of time steps $0,1,2,3, \ldots, n-1$, the following is required.

The scalar measurements are $Z_{0}, Z_{1}, Z_{2}, \ldots, Z_{n-1}$. And they are expressed in followings:

$$
\begin{aligned}
Z_{0} & =H X_{0} \\
Z_{1} & =H X_{1} \\
& =H\left[T X_{0}+\mathbf{u}_{0}\right] \\
& =H T X_{0}+H_{0} \\
Z_{2} & =H X_{2} \\
& =H\left[T\left[T X_{0}+\mathbf{u}_{0}\right]+\mathbf{u}_{1}\right] \\
& =H\left[T^{2} \mathbf{X}_{0}+\mathbf{T} \mathbf{u}_{0}+\mathbf{u}_{1}\right] \\
& =\mathbf{H T}^{2} \mathbf{X}_{0}+\mathbf{H T} \mathbf{u}_{0}+\mathbf{H u}_{1}
\end{aligned}
$$

$$
\begin{aligned}
& \mathrm{Z}_{\mathrm{n}-1}=\mathbf{H} \mathbf{X}_{\mathrm{n}-1} \\
& =\mathbf{H}\left[\mathbf{T}^{\mathrm{n}-1} \mathbf{X}_{0}+\mathbf{T}^{\mathrm{n}-2} \mathbf{u}_{0}+\mathrm{T}^{\mathrm{n}-3} \mathbf{u}_{1}+\mathrm{T}^{\mathrm{n}-4} \mathbf{u}_{2}+\ldots+\mathrm{T} \mathbf{u}_{\mathrm{n}-3}+\mathbf{u}_{\mathrm{n}-2}\right] \\
& =\mathbf{H T}^{\mathrm{n}-1} \mathbf{X}_{0}+\mathbf{H T}^{\mathrm{n}-2} \mathbf{u}_{0}+\mathrm{HT}^{\mathrm{n}-3} \mathbf{u}_{1}+\mathrm{HT}^{\mathrm{n}-4} \mathbf{u}_{2}+\ldots+\mathrm{HT} \mathbf{u}_{\mathrm{n}-3}+ \\
& \mathbf{H} \mathbf{u}_{\mathbf{n}-2}
\end{aligned}
$$

Rearranging the above equations gives: 
$\mathrm{Z}_{0}$

$\mathrm{Z}_{1}-\mathrm{Hu}_{0}$

$\mathrm{Z}_{2}-\mathrm{HT} \mathbf{u}_{0}-\mathrm{Hu}_{1}$
$=\mathbf{H} \mathbf{X}_{0}$

$=\mathrm{HT}^{1} \mathbf{X}_{\mathbf{0}}$

$=\mathbf{H T}^{2} \mathbf{X}_{\mathbf{0}}$

$$
\begin{array}{rr}
Z_{n-1}-H^{n-2} \mathbf{u}_{0}-H^{n-3} \mathbf{u}_{1}-H^{n-4} u_{2}-\ldots-H T & u_{n-3}-H_{n-2} \\
= & H^{n-1} X_{0}
\end{array}
$$

Convert the above group of equations into a single vector-matrix equation :

$$
\mathbf{Z}=\mathbf{E} \mathbf{X}_{\mathbf{0}}
$$

where $\mathbf{Z}$ is formed by stacking up vertically the term on left hand side of each of the equations and $E$ is formed by stacking up vertically $H, H^{1}, H^{2}, H^{3}, \ldots$, and $H^{n}{ }^{n-1}$.

Eq. 6.3 shows that in order to successfully solve for $\mathrm{X}_{0}$ from the series of inputs and scalar measurements, matrix $\mathbf{E}$ must be invertible such that one can compute $X_{0}$ from $Z$, that is:

$$
\mathbf{X}_{0}=\mathbf{E}^{-1} \mathbf{Z}
$$

The invertibility of $\mathbf{E}$ is thus the condition of observability for a state space model. Eq. 6.3 also shows that the observability of a 
system model is dependent on the structures of both the measurement matrix $\mathbf{H}$ and state transition matrix $\mathbf{T}$. The nature of $\mathbf{T}$ is affected directly by the selection of states. The nature of $\mathbf{H}$ is affected by both the selection of states and the design of the measurement system. In practice, when selecting states and designing measurements, the invertibility of $\mathbf{E}$ should be used as a criterion to verify the observability of the state space model.

\section{STOCHASTIC STATE SPACE}

The discussion so far does not consider the issue of uncertainty. Uncertainty is the difference between the behavior of a state space model and its real world counterpart. It involves the noise in the state propagation model and the measurement model. The uncertainty can be represented as a stochastic state space model of the form:

$$
\begin{aligned}
& \mathbf{X}_{t+1}=\mathbf{T} \mathbf{X}_{t}+\mathbf{u}_{t}+\mathbf{w}_{t} \\
& \mathbf{Z}_{\mathrm{t}+1}=\mathbf{H} \mathbf{X}_{\mathrm{t}+1}+\mathbf{v}_{\mathrm{t}}
\end{aligned}
$$

where $w_{t}$ is the model noise and $v_{t}$ is the measurement noise. 


\section{CHAPTER VI}

\section{KALMAN FILTER}

The Kalman filter utilizes the latest available system measurement to update the system variance and optimally estimates the system states. The filter does not perform forecasting. However, it can improve forecast accuracy by providing optimal state estimates of the current time step such that the model is most representative of the real world system as depicted by the latest measurement.

The Kalman filter is used in this study because of its robust characteristics in the sense that the filter algorithm is generally effective regardless to the nature of probability distribution of the uncertainty in the model and measurement (Gelb, 1974). This chapter is a discussion of the pertinent assumptions and characteristics of the Kalman filter. These materials are covered in great depths in many excellent references such as Gelb (1974), Maybeck (1979), Koch (1986), and Anderson, and Burt (1985).

\section{BASIC NOTIONS}

The derivation and structure of the Kalman filter are based on four assumptions : 
1. The system can be represented by a linear model. (Most complex real world systems are non-linear. In such cases linearity can be achieved by first expanding the nonlinear function in Taylor series and then including only terms of first order.)

2. The model and measurement contains white noise.

3. The noise in the model and measurement are Gaussian.

4. The noise in model and measurement are uncorrelated.

The Kalman filter was derived assuming white noise in the model and the measurement. This assumption is not restrictive because a noise containing time-dependence can always be reduced to a white noise using time series models. These associated time series model can then be added to the system model. The assumption regarding the absence of correlation between the model noise and the measurement noise is generally not restrictive, because the source of noise in the two should be unrelated.

The assumption of Gaussian distribution essentially requires that the statistical information of the system noise and the measurement noise be fully conveyed by their first and second moments such that Kalman filter can completely account for their propagations through time. That is, because the Kalman filter algorithm is concerned only with the first and second moments of 
the system noise and the measurement noise. A Gaussian distribution is uniquely characterized by its first and second moments. A Non-Gaussian distribution may require more moments to be uniquely characterized; thus its statistical information may be conveyed by the mean, variance, and moments of higher orders. As long as an adequate amount of statistical information is conveyed by the first and second moments, the Kalman filter would still operate meaning fully.

The assumption of Gaussian noise in the system model and the measurement is not severely restrictive because of the theoretical convenience provided by the Central Limit Theorem which states that the sum of many independent identically distributed distributions of any nature resembles a Gaussian distribution. The noises in the system and measurement in theory could be attributable to a number of sources, the higher the number of sources the more the noise distributions would appear Gaussian.

The Kalman filter algorithm computes an unbiased state estimate based on minimum variance. The Kalman filter is unbiased in the sense that the algorithm is designed such that the mean of the state estimate is equal to the mean of the actual state. Regardless of the nature of the noise distributions, the Kalman filter computes state estimate such that the variance in the state estimate will always be less than or equal to variances of the system and measurement. Kalman filter never causes increase in variance. This characteristic implies that the uncertainty in the system state(s) 
will never increase through the use of the Kalman filter; it will either decrease or stay unchanged.

The Kalman filter is recursive. It utilizes only the latest measurement of the system as it is made available to update the system variance and compute state estimate. It does not re-process any previously utilized measurement. That is because the information from each previously utilized measurement has already been extracted and is "stored" in the filter algorithm in terms of the system variance and state estimate. This recursive property is vital to ensure cost effectiveness in real time operation. A non-recursive filter would require an ever-growing computational task to process and re-process real time hydrologic data which accumulate rather quickly, incrementally slowing down the filtering process.

\section{THREE KINDS OF STATE ESTIMATION PROBLEMS}

The state propagation and estimation procedures can be applied in three different configurations with respect to time and the availability of measurements as shown in Figure 4. Namely, one might update the system states up to the last time step at which measurement is available. This updating is also known as filtering. One might also choose to update the model up to a time step which is some time steps before measurement is last available. This is known as smoothing. Lastly, one might estimate the system states beyond the time step at which measurement is last available. This is called prediction (or forecasting). 
FILTERING

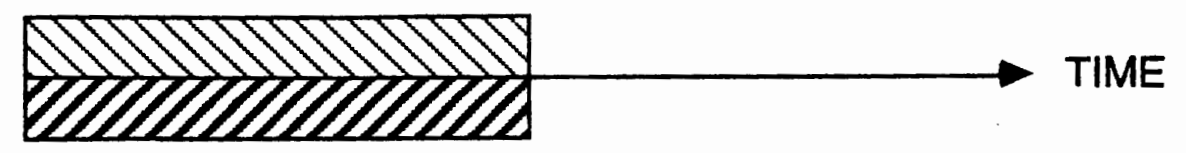

SMOOTHING
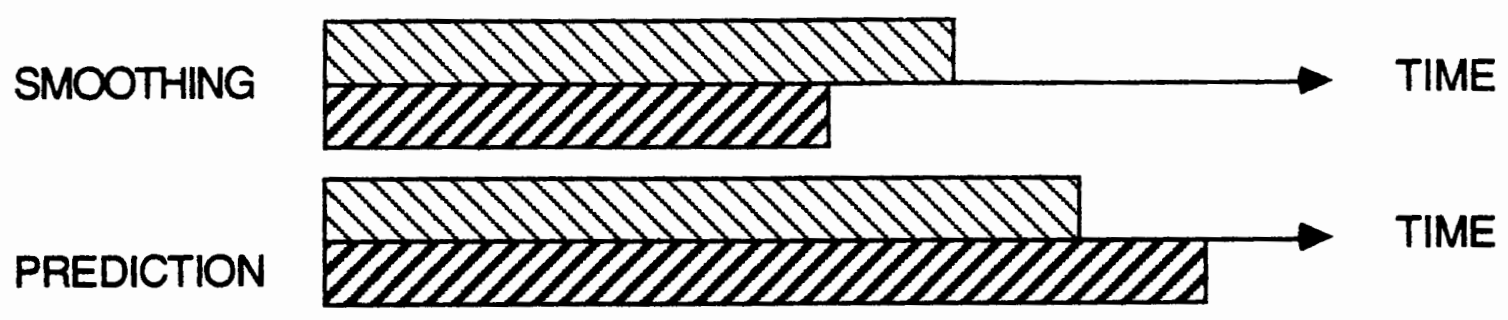

Legend

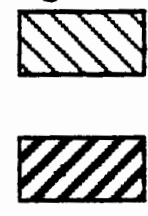

Available Meaurement

Model Estimate

$\longrightarrow$ Time Line

Conducted

Figure 4. Three Applications of Kalman Filter.

In this study, the Kalman filter will be used in filtering the system states. SSARR in state space and a time series model will be used in predicting the system states.

\section{FILTERING IN ONE-DIMENSION: AN ILLUSTRATION}

This example illustrates the basic concepts in the most simplistic case, a one-dimensional estimation problem. Later, this one dimensional concepts will be extrapolated into the general case, filtering in multi-dimensions. Philosophically speaking, the Kalman filter algorithm assumes that all information is useful regardless of its degree of uncertainty and that the degree of usefulness of each information is directly proportional to its uncertainty.

In this illustration, the state occurs in one dimension and is assessed by a system model and by direct measurement. The Kalman 
filter combines the two sources of information and makes an optimal estimate weighting the two by their uncertainties.

Here, information is described by a mean and a variance. Referring to Figure 5, information with less uncertainty $\left(\mathrm{X}_{1}\right)$ has smaller variance and an information with higher variance contains more uncertainty. As shown in Figure 5, the model in general contains more uncertainty than the measurement. The task of the Kalman filter is to estimate the state making use of these two sources of information.

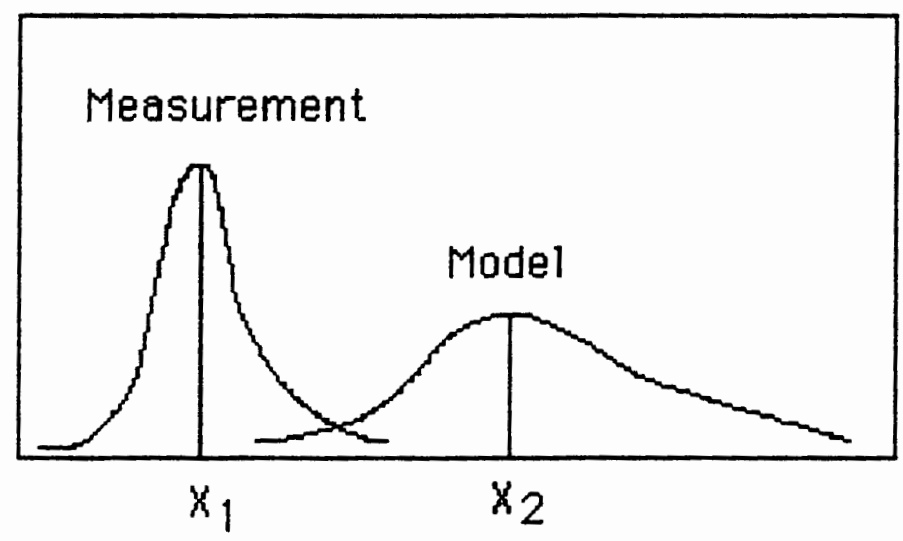

Figure 5. Two Sources of Information.

In one-dimension, the Kalman filter operates by this algorithm:

$$
\mathrm{X}=\mathrm{X}_{1}\left[\mathrm{~V}_{2} /\left(\mathrm{V}_{1}+\mathrm{V}_{2}\right)\right]+\mathrm{X}_{2}\left[\mathrm{~V}_{1} /\left(\mathrm{V}_{1}+\mathrm{V}_{2}\right)\right]
$$

where $X$ is the optimal state estimate; $V_{1}$ is the variance of the state estimated by the forecast model; $\mathrm{X}_{1}$ is the state estimated by the forecast model; $V_{2}$ is the variance of the 
state estimated by the measurement; and $\mathrm{X}_{2}$ is the state estimated by the measurement.

Note that the mean of the optimal estimate $\mathrm{X}$ is closer to the mean of the better (less uncertain) information source, in this cast the measurement.

Based on EQ 6.1, one can derive the variance (V) of the optimal state estimate of $\mathrm{X}$ to be:

$$
V=\left[V_{2} /\left(V_{1}+V_{2}\right)\right]^{2} V_{1}+\left[V_{1} /\left(V_{1}+V_{2}\right)\right]^{2} V_{2}
$$

where $\mathrm{V}$ is the variance of the optimal state estimate.

Note that $\left[\mathrm{V}_{2} /\left(\mathrm{V}_{1}+\mathrm{V}_{2}\right)\right]$ is always less than 1 . Thus $\mathrm{V}$ is always less than $V_{1}$ and $V_{2}$. That is, the variance (uncertainty) of the optimal estimate is always less than the variances of the model prior to filtering and the measurement. This illustrates the minimum variance nature of Kalman filter, displayed graphically in Figure 6. 


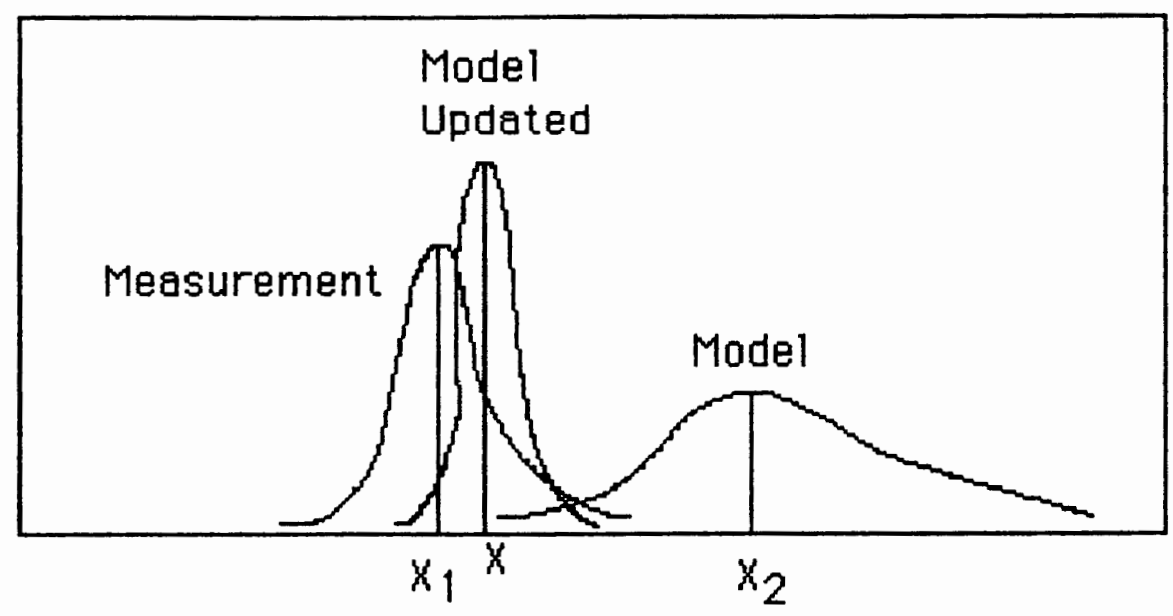

Figure 6. Model Update

Furthermore, using Kalman filter, one can compute the reduction in uncertainty (i.e. Variance, V) :

$\mathrm{V}=\mathrm{V}_{1}-($ reduction in $\mathrm{V})$

So the reduction in $\mathrm{V}$ is $\mathrm{V}_{1}-\mathrm{V}$ or

$$
\mathrm{V}=\mathrm{V}_{1} 2 /\left(\mathrm{V}_{1}+\mathrm{V}_{2}\right)
$$

Thus, the uncertainty of the one-dimensional model is reduced by $\mathrm{V}_{1} 2 /\left(\mathrm{V}_{1}+\mathrm{V}_{2}\right)$ when the measurement is "filtered into the model". This shows that the reduction in uncertainty is minimal but always larger than zero when the measurement very uncertain (i.e. $V_{2}$ is large). Likewise, when measurement is very certain (i.e. $V_{2}$ is very small), uncertainty of the model is reduced by an amount approaching $\mathrm{V}_{1}$, meaning that the uncertainty in the model is 
essentially eliminated. (When $V_{2}$ equals $V_{1}$, the reduction in $\mathrm{V}$ is $\mathrm{V}_{1} / 2$, meaning that the uncertainty in the model is reduced by one half.) No matter how large $V_{2}$ is, the uncertainty of the model is always reduced by the filter.

From the standpoint of modeling in real time, the optimal state will be corrupted by the forecast model when another forecast is made. This corruption is due to the uncertainty inherent in goodness of fit of the model and the uncertainty in the deterministic input to the model. Thus, the variance of the state will increase. When measurement is available, this new state forecast will be filtered and its variance will once again decrease. This increasethen-decrease in the state variance through the course of forecasting and filtering is typical in real time modeling using a forecast model and Kalman filter (Figure 7.).

Usually, forecasting and filtering begins with some almost arbitrary initial condition of the states which is usually a very uncertain estimate of the state. The state uncertainty steeply decreases when a measurement is available and filtering is performed. In subsequent time steps, the state variance may stabilize in a way that it fluctuates up and down within a fixed range of amplitude through the forecast and filtering activities. 

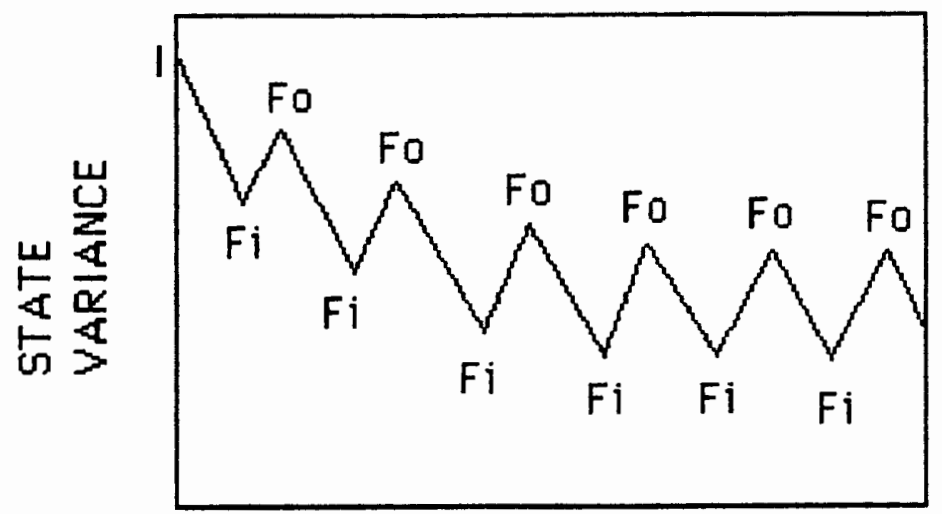

Legend

TIME

$1=$ initial condition

$\mathrm{Fi}=$ Filtering

Fo $=$ Forecast

Figure 7. Changes of State Variance Through Time. (Note the increase and decrease of variance through the course of forecasting and filtering.)

\section{KALMAN FILTER ALGORITHM IN ONE-DIMENSION}

Eq 6.1 and 6.2 conveyed the conceptual essence of Kalman filter. Suppose the one dimension system discussed above is autoregressive and the state is directly measured. Utilizing the basic ideas embedded in $\mathrm{Eq} 6.1$ and 6.2, the following onedimensional filtering algorithm can be created, of which the multidimensional algorithm is simply an extension.

1. The state model is:

$$
\mathrm{X}_{\mathrm{k}}=\mathbf{a} \mathrm{X}_{\mathrm{k}-1}+\mathrm{w}_{\mathrm{k}-1}
$$


where $X$ is the state of the system; a is the autoregressive coefficient; $w$ is the white noise in system model; and $\mathrm{k}$ is time step.

2. The measurement model is:

$\mathrm{Z}_{\mathrm{k}}=\mathrm{X}_{\mathrm{k}}+\mathrm{v}_{\mathrm{k}}$

where $v$ is the white noise in measurement and $Z_{k}$ is the measurement of the system for time step $k$.

3. The state estimate extrapolation is performed by:

$\mathrm{X}_{\mathrm{k}}(-)=\mathrm{a} \mathrm{X}_{\mathrm{k}-1}(+)$

where $\mathrm{X}_{\mathbf{k}}(-)$ is the state estimate of time step $\mathrm{k}$ without filtering and $\mathrm{X}_{\mathrm{k}-1}\left(^{+}\right)$is the optimal state estimate of time step $k-1$ after filtering

4. The error variance extrapolation is:

$\mathrm{P}_{\mathrm{k}}(-)=\mathrm{a}^{2} \mathrm{P}_{\mathrm{k}-1}(+)+\mathrm{Q}_{\mathrm{k}-1}$

where $\mathrm{P}_{\mathrm{k}}(-)$ is the system variance of time step $\mathbf{k}$ before filtering; $\quad \mathrm{P}_{\mathrm{k}-1(+)}$ is the optimal system variance of time step $\mathrm{k}-1$ after filtering; and $\mathrm{Q}_{\mathrm{k}-1}$ is the variance of system model noise at time step $\mathrm{k}-1$. 
5. Kalman Gain is computed as the Kalman gain matrix, $\mathrm{K}_{\mathrm{k}}$, a algebraic term that accounts for the gain in information by filtering the measurement into the model:

$\mathrm{K}_{\mathbf{k}}=\mathrm{P}_{\mathbf{k}}(-) /\left(\mathrm{P}_{\mathbf{k}}(-)+\mathrm{R}_{\mathbf{k}}\right)$

where $\mathrm{K}_{\mathrm{k}}$ is the Kalman Gain of time step $k$ and $R_{k}$ is the variance of measurement noise of time step $k$.

Note that the computation of the Kalman gain matrix is conceptually equivalent to the Eq. 6.2.

6. The optimal state estimate by filtering is given by:

$\mathrm{X}_{\mathrm{k}}(+)=\mathrm{X}_{\mathrm{k}}(-)+\mathrm{K}_{\mathrm{k}}\left[\mathrm{Z}_{\mathrm{k}}-\mathrm{X}_{\mathrm{k}(-)}\right]$

Note that this filtering step is essentially equivalent to Eq. 6.1.

7. The optimal error variance by filtering is given by:

$\mathrm{P}_{\mathrm{k}}(+)=\left[\begin{array}{lll}1-\mathrm{K}_{\mathrm{k}} & \mathrm{P}_{\mathrm{k}}(-)\end{array}\right.$

Note that this filtering step is again essentially equivalent to Eq. 6.2. 


\section{FILTERING IN MULTI-DIMENSIONS - THE GENERAL PROBLEM}

The above illustration shows how the Kalman filter works in one-dimension. The Kalman filter algorithm is written using vectors and matrices because system models generally occur in multi-dimension such that the state occurs as a state vector and variance occurs as a covariance matrix. The weighting process that gives rise to the optimal state estimate is embedded in Kalman gain matrix, which is computed based on system measurement and covariance matrix of the system model.

A covariance matrix contains variances of the states in the diagonal elements and covariances of the states in the off-diagonal elements. Thus the covariance matrix does not only contain information regarding the variability (or uncertainty) of the states alone; it also contains information regarding the correlations among the states.

The Kalman filter weighs two sources of the information of each particular state. In general, with a multi-variate system model, each state is either related to the measurement by a function or the state is not measured in anyway at all. In the former case, the Kalman filter will compute the state estimate using both the deterministic information conveyed in the measurement matrix and the statistical information conveyed in the system covariance matrix. In the latter case, the Kalman filter will compute the state estimate using only the statistical information. 


\section{KALMAN FILTER ALGORITHM IN MULTI-DIMENSIONS}

The filtering algorithm in multi-dimensions is completely analogous to the one-dimensional algorithm discussed earlier. The following is a summary of the seven basic components in the algorithm in multi-dimensions.

\section{System Model in State Space Form}

In order to apply the Kalman filter at all, the system must first be represented in a linear state space format. The system model discussed here is time invariant such that $T, I$, and $H$ are constant and do not need to be subscripted by time step. However these following equations do apply to time-variant system. In such cases, $\mathbf{T}, \mathbf{I}$, and $\mathbf{H}$ do vary through time and need to be subscripted with time step. The model is represented by:

$$
\begin{aligned}
& \mathbf{X}_{\mathbf{k}}=\mathbf{T} \mathbf{X}_{\mathbf{k}-1}+\mathbf{F} \mathbf{U}_{\mathbf{k}-1}+\mathbf{w}_{\mathbf{k}-1} \\
& \mathbf{Z}_{\mathbf{k}}=\mathbf{H} \mathbf{X}_{\mathbf{k}}+\mathbf{v}_{\mathbf{k}}
\end{aligned}
$$

where $\mathbf{X}_{k}$ is the state vector of time step $k$. The variability of this state vector is characterized by the state covariance matrix $P_{k} ; T$ is the time invariant state transition matrix; $X_{k-}$ 1 is the state vector of time step $k-1$. The variability of this state vector is characterized by the state covariance matrix $\mathbf{P}_{\mathbf{k}-1} ; \mathbf{F}$ is the input matrix; $\mathbf{U}_{\mathbf{k}-1}$ is the input vector; $\mathbf{w}_{\mathrm{k}-1}$ is the model noise of time step $k-1$. It is characterized by 
covariance matrix $Q_{k} ; Z_{k}$ is the measurement vector of time step $\mathbf{k}$; and $\mathbf{v}_{\mathbf{k}}$ is the measurement noise of time step $k$. It is characterized by covariance matrix $\mathbf{R}_{\mathbf{k}}$.

As discussed earlier, the derivation of the Kalman filter uses several assumptions on the stochastic properties of the system :

a. The model noise, $\mathbf{w}_{\mathbf{k}}$, is normally distributed and zero mean.

b. The measurement noise, $\mathbf{v}_{\mathrm{k}}$, is normally distributed and zero mean.

c. The model noise, $\mathbf{w}_{\mathbf{k}}$, and measurement noise, $\mathbf{v}_{\mathbf{k}}$, are uncorrelated.

When these assumptions are violated, the performance of the filter will be suboptimal.

\section{Initial Condition of the System Model}

The model must be given an initial condition, before any forecast or filtering is conducted. Unless an initial condition is known to be accurate, the user assumes some arbitrary initial condition and assigns to it a noise covariance matrix, $\mathbf{P} 0$, of very high magnitude. Thus, once the forecast and filtering sequence is started, the real measurement will be used to guide the system model from the initial state of high uncertainty to reasonable certainty. This will occur automatically in the filtering process. 
The initial condition of the state is thus a state vector of numbers that the user assigns based on engineering judgment if no more accurate means of estimation is available. The initial state vector is defined here as $\mathrm{X}^{\prime} 0(+)$, which indicates that the state vector is an estimate, the subscript 0 indicates that the vector is at initial time, and the $(+)$ indicates that the state vector is the estimate of this time step and is now ready to be propagated.

\section{State Estimate Extrapolation}

The state extrapolation procedure is to propagate the state vector of the system from one time step using the input and the system structure conveyed in the state transition matrix. In other words, this is the forecast step from time step $k-1$ to time step $k$. The propagation (or forecast) is conducted be the equation:

$$
\mathbf{X}_{\mathbf{k}}^{\prime}(-)=\mathbf{T} \mathbf{X}_{\mathbf{k}-1}(+)+\mathbf{F} \mathbf{U}_{\mathbf{k}-1}
$$

where $X^{\prime} k(-)$ is the state vector forecast for time step $k$; $T$ is the state transition matrix; $\mathbf{X}_{\mathrm{k}-1\left(^{+}\right)}$is the state vector estimate; $F$ is the input matrix. It controls how the input vector affects the states; and $\mathbf{U}_{\mathbf{k}-1}$ is the input vector.

Note that the difference between a state vector forecast and a state vector estimate is that the forecast is computed using only the propagation model but the estimate is computed utilizing both the model and measurement. 


\section{State Error Covariance Extrapolation}

The error in the states due to model noise is accounted for in the dynamic system model propagating the model covariance matrix. Note that the difference between a state noise covariance matrix forecast and a state noise covariance matrix estimate is that the forecast is computed using only the propagation model but the estimate is computed utilizing both the model and measurement.

The propagation of state noise is analogous to the propagation of the states. The propagation of uncertainty among the states is driven by that same mechanism the drives the states and is computed as:

$$
\mathbf{P}_{\mathbf{k}}(-)=\mathbf{T} \mathbf{P}_{\mathbf{k}-1(+)} \mathbf{T}+\mathbf{Q}_{\mathbf{k}-1}
$$

where $\mathbf{P}_{\mathbf{k}}(-)$ is the state noise covariance matrix forecast of time step $\mathbf{k} ; \mathbf{T}$ is the state transition matrix; $\mathbf{P}_{\mathbf{k}-1}\left(^{+}\right)$is the state noise covariance matrix estimate of time step $k-1$; and $Q_{k-1}$ is the model noise covariance matrix of time step $k-1$.

\section{Kalman Gain Matrix}

The Kalman gain matrix, $K$, contains all the information needed for estimating the states and the associated uncertainty in the system, making an optimal compromise between the information provided by the model forecast and the information provided by system measurement. Note that the computation of Kalman gain matrix does not require a large and growing database containing 
information of the past behavior of the system. Kalman filtering is a recursive process. New information is processed and synthesized recursively such that the state noise covariance matrix will at each time step be updated. This covariance matrix at each time step contains the latest updated information of the uncertainty of the system states.

The Kalman gain matrix controls the adjustment of the state vector such that each state in the state vector is adjusted based on:

a. The covariances (and variances) relating the particular state and the states that are used in computing the forecast,

b. the weighting coefficients in the measurement matrix that form the linear combination of the states for generating the forecast, and

c. the observation noise vector of time step $\mathbf{k}$.

The optimal estimate of the state vector and the state covariance matrix is subsequently controlled by the values of Kalman gain matrix which is computed at each time step as:

$$
\mathbf{K}_{\mathbf{k}}=\mathbf{P}_{\mathbf{k}(-)} \mathbf{H}^{\mathrm{T}}\left[\mathbf{H} \mathbf{P}_{\mathbf{k}(-)} \mathbf{H}^{\mathrm{T}}+\mathbf{R}_{\mathbf{k}}\right]^{-1}
$$

where $\mathbf{K}_{\mathbf{k}}$ is the Kalman gain matrix of time step $\mathbf{k} ; \mathbf{P}_{\mathbf{k}}(-)$ is the system state noise covariance matrix forecast; $H^{T}$ is the 
transpose of observation matrix; and $\mathbf{R}_{\mathbf{k}}$ is the observation noise vector of time step $k$.

\section{State Estimate Update}

At each time step $\mathbf{k}$, the state forecast, $\mathbf{X}_{\mathbf{k}} \mathbf{( - )}$, is only a product of the state transition model. The measurement of $k$, when available in real time, is utilized to update the state forecast, producing the state estimate $\mathrm{X}_{\mathrm{k}}^{\prime}(+)$, using the following equation :

$$
\mathbf{X}_{\mathbf{k}}^{\prime}(+)=\mathbf{X}_{\mathbf{k}}^{\prime}(-)+\mathbf{K}_{\mathbf{k}}\left[\mathbf{Z}_{\mathbf{k}}-\mathbf{H} \mathbf{X}_{\mathbf{k}(-)}^{\prime}\right]
$$

Substituting $\mathbf{X}_{\mathbf{k}(-)}^{\prime}=\mathbf{T} \mathbf{X}_{\mathbf{k}-1}^{\prime}(+)-F \mathbf{U}_{\mathbf{k}-1}$, gives:

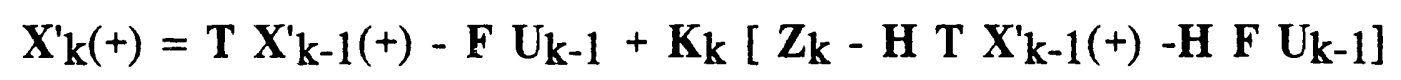

This equation illustrates the discrepancy between the forecast observation and the measured observation. This discrepancy is weighted by the Kalman Gain Matrix and added to the state forecast. For each state in the state vector, the resulting adjustment could be positive or negative. $\mathrm{X}_{\mathbf{k}}^{\prime}(+)$ is the optimal compromise between the state forecast model and the real observation after the noise associated with the two are weighted.

\section{State Error Covariance Estimate Update}

In the same way the state are propagated and updated through each time step, the noise associated with the states, $P$, are also 
propagated and updated. The state covariance matrix estimate, as opposed to the forecast, is computed by the following equation :

$$
\mathbf{P}_{\mathbf{k}}(+)=\left[\mathbf{I}-\mathbf{K}_{\mathbf{k}} \mathbf{H}\right] \mathbf{P}_{\mathbf{k}}(-)
$$

where $\mathbf{P}_{\mathbf{k}}(+)$ is the system state noise covariance matrix estimate; $\mathbf{P}_{\mathbf{k}}(-)$ is the System State Noise Covariance Matrix forecast; $I$ is the identity matrix; $K_{k}$ is the Kalman gain matrix at time step $k$; and $H$ is the observation matrix.

\section{THE SEQUENCE OF EVENTS IN A REAL TIME MODELING/MONITORING SCENARIO}

Once a state space model and the mechanism to measure the real system are established, the system model is ready to be used in real time modeling, in the following sequence of steps :

\section{Step 1: Set initial condition}

At time step 0 , define initial state values based on judgment. Assign state noise covariance with values of high magnitude in comparison to the noise covariance associated with the model and measurement.

Step 2: Compute state vector and state noise covariance forecasts

At time step 0 , propagate state from time step 0 to 1 using; 


$$
\mathbf{X}^{\prime} 1(-)=\mathbf{T} \mathbf{X}_{0}(+)+\mathbf{F} \mathbf{U}_{0}
$$

Also propagate state noise covariance matrix from time step 0 to 1 using:

$$
\mathbf{P}_{1}(-)=\mathbf{T} \mathbf{P}_{0}(+) \mathbf{T}+\mathbf{Q} 0
$$

\section{Step 3: Obtain system measurement}

At time step $1, \mathrm{Z}_{1}$ is made available through the measuring mechanism, such as telemetric streamflow gauges and other hydrologic models as is the case when monitoring the Mehama Local Basin.

Step 4: Compute Kalman gain matrix

At time step 1, perform the computation:

$$
\mathbf{K}_{1}=\mathbf{P}_{1(-)} \mathbf{H}^{\mathrm{T}}\left[\mathbf{H}_{\mathbf{1}} \mathbf{P}_{1}-\mathbf{H}^{\mathrm{T}}+\mathbf{R}_{1}\right]^{-1}
$$

Step 5: Compute state vector and state noise covariance estimates

At time step 1, update state vector using the measurement and the Kalman gain matrix as:

$$
\mathbf{X}_{1}^{\prime}(+)=\mathbf{X}_{1}^{\prime}(-)+\mathbf{K}_{1}\left[\mathbf{Z}_{1}-\mathbf{H} \mathbf{X}^{\prime} 1(-)\right]
$$


Update the state noise covariance matrix using the measurement and the Kalman gain matrices by:

$$
\mathbf{P}_{1}(+)=\left[\mathbf{I}-\mathbf{K}_{1} \mathbf{H}\right] \mathbf{P}_{1}(-)
$$

Repeat steps 2 through step 5 for each time steps in real time. 


\section{CHAPTER VII}

\section{COMPUTATIONAL EXPERIMENT \\ OVERVIEW- BASIC SCHEME AND ASSUMPTIONS \\ OF THE EXPERIMENT}

The background concepts of modeling in general, time series modeling and the Kalman filter have been discussed. This chapter is a presentation of how these concepts were put to use in an experiment, in conjunction with a deterministic hydrologic model, the SSARR Mehama Local Basin Model. The historic residuals of this model (discrepancy between the historic model forecasts and the corresponding historic streamflow measurements) were analyzed and used to achieve better model forecast accuracy. This experiment is a first attempt. Its purpose is to establish a "base case" illustration as to how time series analysis and the Kalman filter can be applied to SSARR in real time forecasting. That is, this base case is intended to adopt the most simplistic route to solving the problem. The solution achieved herein, being the base case, may not necessarily contribute to the operation at RFC immediately. However, it will serve the purpose of providing a starting-point and a direction with which more sophisticated solutions can be developed in the future. The Mehama Local Basin Model was chosen as the subject model at the recommendation of RFC Senior Hydrologist 
Dr. Vernon Bissell. While this model has the typical structure of all other SSARR models, this model also belongs to a category of models that are rather uncertain in the sense that they are measured indirectly based on mathematical inference using hydrologic data of nearby basins and river reaches.

In this experiment, when analyzing the stochastic characteristics of the SSARR Mehama Local Basin Model, the assumption was made that the measurement, however indirect, represents the true behavior of the local basin. This assumption implies that the "measured" streamflow is assumed to be practically without noise. It also implies that the model must be corrected to reflect the measurement whenever it disagrees with the measurement.

The state space formulation used here is for only the storages in the routing phases for a single time step. These routing phases are responsible for uniquely computing the streamflow hydrograph. This state space form involves only 9 states and is linear. The author believes that this is the simplest state space form for the local basin model. This state space form is observable when checked by the conditions defined by the measurement matrix, $\mathbf{H}$, and the state transition matrix, $\mathrm{T}$.

More sophisticated state space forms inevitably require more states; one state space form, suggested by Dr. Vernon Bissell of the RFC, requires about 84 states. Briefly, the formulation involves a structure that incorporates states of several time steps expressed as functions of states of some initial time step (as many as 7 was 
proposed by Dr. Bissell). This formulation is designed to estimate the states at an initial time steps using the streamflow and moisture input measurements of the initial as well as several subsequent time steps. The inclusion of moisture accounting terms makes the model non-linear. This 84-state state space form is not necessarily more effective than the simplistic base-case formulation in achieving the goals of optimal filtering. This is due to two observations:

1. This form involves a non-linear model due to the inclusion of the moisture accounting term and, unless the Extended Kalman filter is applied, the model needs to be linearized by approximation using the Taylor Expansion. Such an expansion would considerably increase the uncertainty in the model.

2. Preliminary derivation indicated that the algebraic formulation and computer coding required for this form were extremely tedious and lengthy. Early in its development, it became obvious that the development of this state space form would greatly exceed the research funding and time available for this study.

The development of this 84-state state space form was promptly discontinued upon these two observations. The simplistic 9-state form was adopted instead. 
Computational experiments were carried out by a battery of original FORTRAN and Pascal programs that interfaced with the existing software and database at RFC. These programs were run on the AMDALH mainframe used by RFC and a Macintosh personal computer.

\section{DATA}

The basic data for this computational experiment were for the four early winter months of 1982, October 1 through December 31 . Dr. Bissell generated these data when he reproduced the operation of Mehama Local Basin for that period at 6-hourly time steps. The following procedure was used to generate the data:

1. To generate the model "forecast" for the local basin, he ran Mehama Local Basin using historic record of temperature and moisture inputs. The moisture input time series is shown in Figure 8.

2. To generate the "measurement" for the local basin, the routed Big Cliff streamflow were subtracted from the Mehama outflow. The time series is shown in Figure 9. 


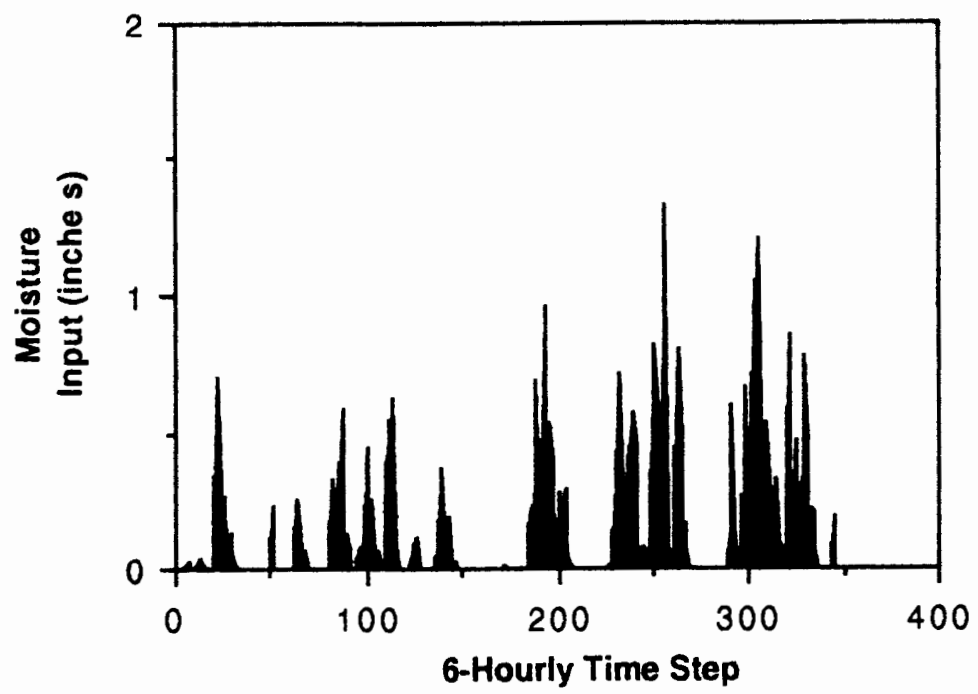

Figure 8. Moisture input to Mehama Local Basin 0000 hour October 1 to 2400 hour December 31, 1982.

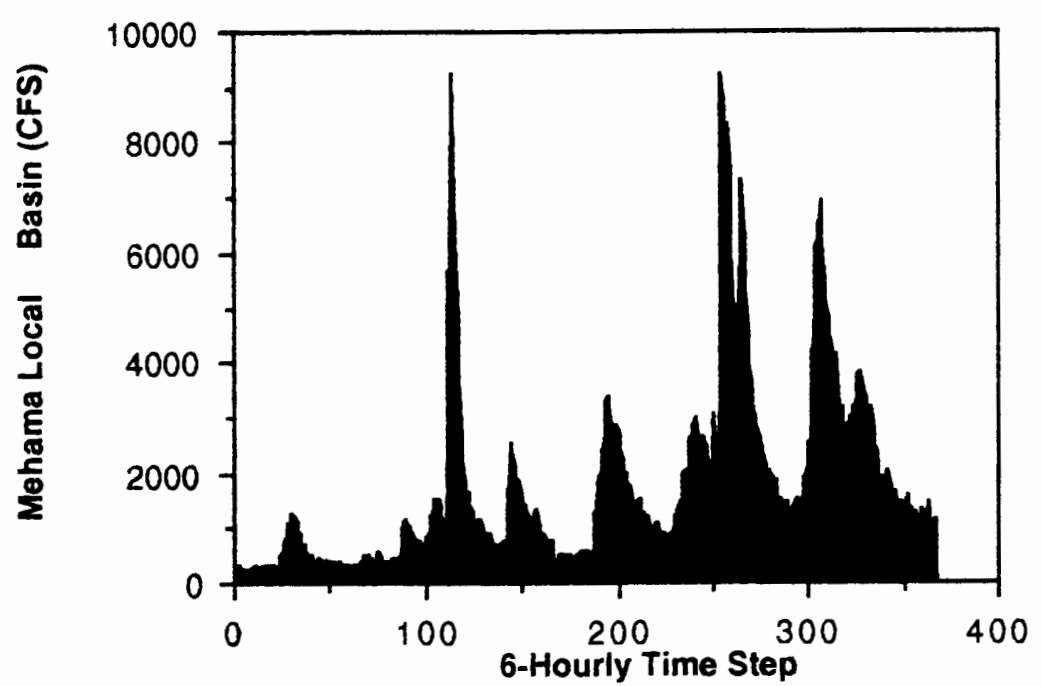

Figure 9. "Measured" Streamflow of Mehama Local Basin 0000 hour October 1 to 2400 hour December 31, 1982. 
3. Evapotranspiration data applicable for the project area were determined by Dr. Bissell. The monthly average evapotranspiration loss in inches, beginning with January and ending with December, was $0.15,0.2,0.3,0.5,0.6$, $0.8,0.9,1.0,0.8,0.6,0.3,0.15$.

The error and error-squared time series are shown in Figures 10 and 11 respectively. The error series was generated by subtracted the modeled streamflow from the measured streamflow. This error series was the source of information regarding the stochastic nature of the model-measurement system. After the stochastic modeling work, any structure of this error time series was extracted and utilized to ultimately reduce the variance of the error time series.

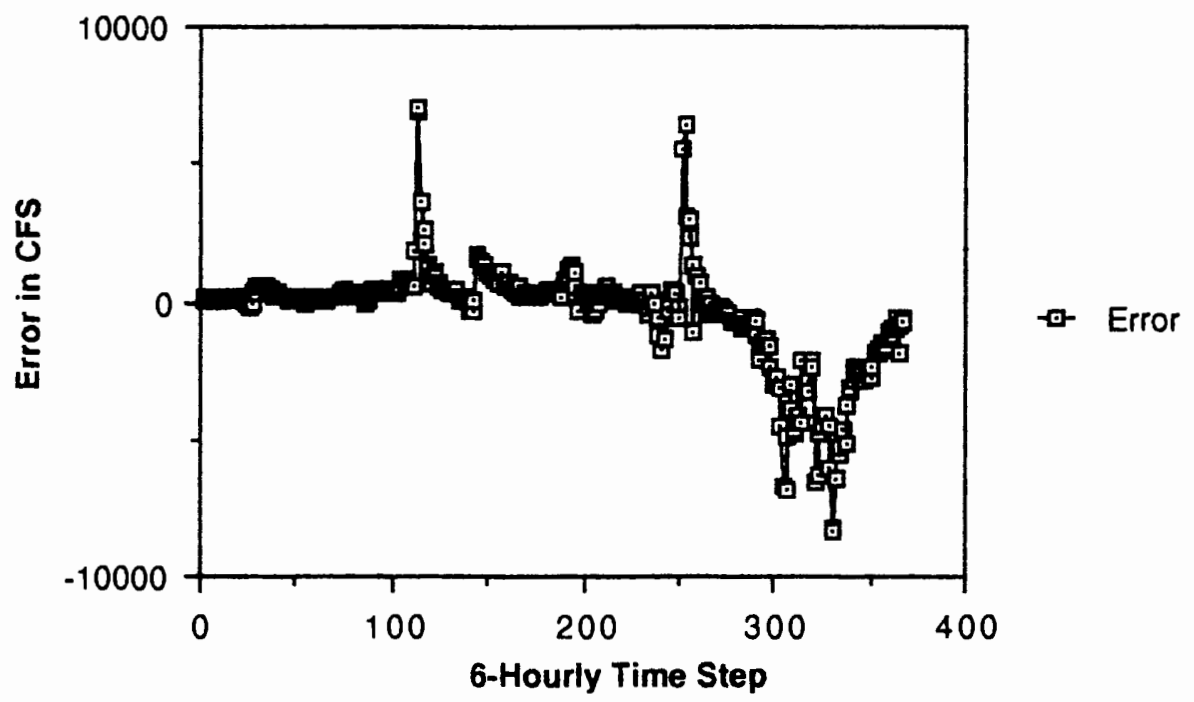

Figure 10. Mehama Local Basin Model Error 0000 hour October 1 to 2400 hour December 31, 1982. 


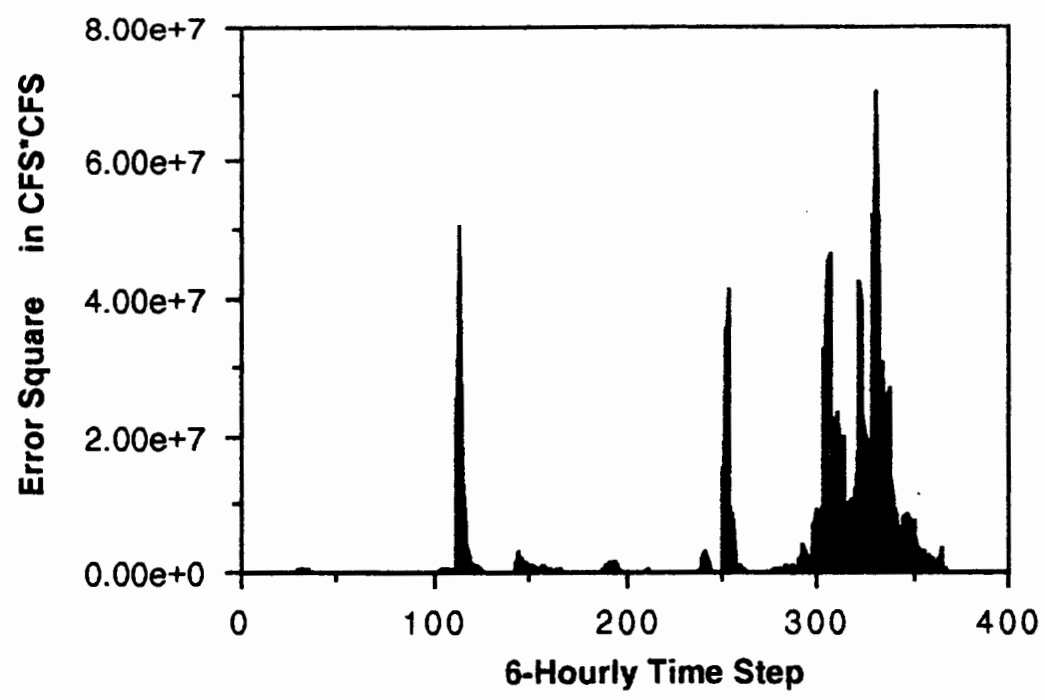

Figure 11. Residual-Squared Time Series, 0000 hour October 1 to 2400 hour December 31, 1982.

Figures 8 through 11 illustrate the following:

1. The local basin responds to the moisture input on an event-by-event basis. The rising limbs of the response hydrographs are sharp and abrupt, while the falling limbs are, in general, more gradual.

2. The peak flows of the hydrographs are proportional to the antecedent condition of the watershed in the sense that higher peaks are achieved when moisture input occur through a cluster of time steps. When moisture input does not occur in a cluster of time steps, the watershed is allowed to dry and subsequent moisture input does not cause as high a hydrograph peak. 
3. The error squared time series is strongly correlated with the moisture input series. This suggests that the uncertainty in SSARR Mehama Local Basin Model is attributable to moisture input. When moisture input is low, the model tends to make a better forecast.

Observation 1 and 2 are consistent with the usual response of a watershed to moisture inputs.

\section{MODEL RESIDUAL (NOISE)}

Using historic precipitation and temperature data, the SSARR Mehama Local Model, and the appropriate gaged river flow data of $\mathrm{N}$. Santiam River for the period from the 0000 hour on October 1 to the 2400 hour on December 31,1982, the residual time series was generated by the equation :

\section{Residualt $=$ MLBC $_{t}-$ MLBMV $_{t}$}

where $\mathrm{t}$ is the time step; MLBC is the Mehama Local Basin Outflow; MLBMV is the Mehama Local Basin Outflow generated by the SSARR Model

The Mehama Local Basin Outflow, MLBC, was computed by this equation : 
$\mathrm{MLBC}_{\mathrm{t}}=\mathrm{MGO}_{\mathrm{t}}-\mathrm{RR}[\mathrm{BIGC}]_{\mathrm{t}}$

where MGO is the Mehama gaged outflow; BIGC is the Big Cliff gaged outflow; and RR[BIGC] is the Big Cliff gaged outflow routed through a reach of $N$. Santiam River by a SSARR river routing model;

The residual time series was computed as 6-hour long time steps for October, September, and November of 1982. The resulting residual time series consisted of 368 data points. Its statistical characteristics are shown in Table $I$.

The residual time series was then normalized by this equation: $\mathrm{Z}=(\mathrm{X}-\mathrm{Mean}) /($ Standard Deviation $)$

\section{ESTIMATING A TIME SERIES MODEL}

Several candidate models were computed for the residual time series, using the algorithm discussed earlier. They are summarized in Table II where " $\mathrm{m}$ " terms are MA parameters and "a" terms are AR parameters. 
TABLE I

STATISTICAL CHARACTERISTICS OF THE RESIDUAL TIME SERIES MEHAMA LOCAL BASIN MODEL

Characteristic

Mean

Sum of Squares

Estimated Variance

Estimated Standard Deviation

Estimated Skewness
Value

-397.85 CFS

1309423000 CFS $^{2}$

$3.6 \times 10^{6}$

1888.89

$-1.027$

(i.e. skewed to the right)

TABLE II

TIME SERIES ESTIMATION RESULTS

Model

$\operatorname{AR}(1)$

$\operatorname{AR}(2)$

$\operatorname{AR}(3)$

$\operatorname{AR}(4)$

$\operatorname{ARMA}(1,1)$

$\operatorname{ARMA}(1,1)$
Parameters

$\mathrm{a} 1=0.934$

$\mathrm{a}_{1}=1.1100, \mathrm{a}_{2}=-0.1899$

$\mathrm{a}_{1}=1.1739, \mathrm{a}_{2}=-0.5657$ a3 $=0.3396$

$\mathrm{a}_{1}=1.1779, \mathrm{a}_{2}=-0.5723$

$\mathrm{a} 3=0.3533, \mathrm{a} 4=-0.0116$

$\mathrm{a}_{1}=0.88608, \mathrm{~m}_{1}=-0.38992$

$\mathrm{a}_{1}=0.9398, \mathrm{~m}_{1}=-0.1702$
Estimation

Procedure

Regression

Regression

Regression

Regression

Direct 2-D

Search

Inferred from $\operatorname{AR}(2)$ 
TABLE II

TIME SERIES ESTIMATION RESULTS

(continued)

Model

$\operatorname{ARMA}(2,1)$

$$
\begin{aligned}
& \mathrm{a}_{1}=-0.4819, \mathrm{a}_{2}=0.0000 \\
& \mathrm{~m}_{1}=0.6920
\end{aligned}
$$

$\operatorname{ARMA}(2,2)$

$$
\begin{aligned}
& \mathrm{a}_{1}=0.7476, \mathrm{a}_{2}=0.2573 \\
& \mathrm{~m}_{1}=-0.4303, \mathrm{~m}_{2}=0.3227
\end{aligned}
$$

$\operatorname{ARMA}(3,1)$

$$
\begin{aligned}
& \mathrm{a}_{1}=1.2107, \mathrm{a}_{2}=-0.6109 \\
& \mathrm{a}_{3}=0.3721, \mathrm{~m}_{1}=0.0328
\end{aligned}
$$

Estimation

Procedure

Inferred

from

$\operatorname{AR}(3)$

Inferred from

AR(4)

Inferred from

AR(5)

These candidate models all produce white noise residual (based on Porte Manteau Test) and satisfy invertibility conditions. Not every model was checked for stationary conditions. The AR(3) was determined to be non-stationary and it is assumed that AR(4) was non-stationary too, because its model parameters differ very little from the parameters of $\operatorname{AR}(3)$.

For comparison, the characteristics of the models were computed and are displayed in Table III. 
TABLE III

CHARACTERISTICS OF CANDIDATE MODELS

Note : $\quad \begin{array}{ll}\mathbf{N} & =\text { number of data points; } \\ \mathbf{k} & =\text { number of parameters in model; } \\ \text { AIC } & =\text { Akaike Information Criterion for parsimony }\end{array}$

Sum of Square Correlation

Model $\underline{\mathrm{N} / \mathrm{k}} \quad \underline{\mathrm{AIC}}$ of Residual $\underline{\text { of Residual }}$

$\underline{(\mathrm{Lag}=1)}$

$\begin{array}{lccll}\operatorname{AR}(1) & 368 & -754.7 & 47.0739 & 0.1764 \\ \operatorname{AR}(2) & 184 & -766.1 & 45.3909 & 0.0643 \\ \operatorname{AR}(3) & 123 & -809.4 & 40.1356 & 0.0036 \\ \operatorname{AR}(4) & 92 & -807.5 & 40.1317 & 0.0003 \\ \operatorname{ARMA}(1,1) & 184 & -792.7 & 43.23 & -0.0563 \\ \operatorname{ARMA}(2,1) & 123 & -735.4 & 49.0751 & -0.1697 \\ \operatorname{ARMA}(2,2) & 92 & -760.0 & 45.65 & -0.0505 \\ \operatorname{ARMA}(3,1) & 92 & -805.6 & 40.33 & -0.0504\end{array}$




\section{TIME SERIES MODEL AUGMENTING MEHAMA LOCAL BASIN MODEL}

All of the eight candidate models satisfy invertibility conditions and are white-noise-able. $\operatorname{AR}(1), \operatorname{AR}(2), \operatorname{ARMA}(1,1)$, $\operatorname{ARMA}(2,1)$, and $\operatorname{ARMA}(2,2)$ are stationary. $\operatorname{ARMA}(3,1)$ and $\operatorname{AR}(3)$ are proven to be non-stationary. $\mathrm{AR}(4)$ is likely to be non-stationary because its parameters are similar to $\operatorname{AR}(3)$. Non-stationary models are controversial because they may be unstable from an computation standpoint and the data record is too short to meaningfully convey any seasonality or cyclicity. Among the stationary models and judging by the number of model parameters (and thus operation cost), the sum of square, the value of "N/k", the residual correlation, and Akaike Information Criteria, the $\operatorname{ARMA}(1,1)$ is the most effective.

\section{MEHAMA LOCAL BASIN MODEL IN STATE SPACE}

The state space formulation used here was designed to include only discharges in the routing phases as states. The moisture accounting portion of SSARR, including the "splitting functions", was excluded. This decision is based on the facts that the streamflow hydrograph is fully described by the routing phases and that SSARR does not forecast moisture input. The SSARR Mehama Local Basin model has no provision for forecasting future moisture input and thus the estimation of future moisture input is strictly a measurement task based on processing hydrometeorological data outside the SSARR model. 
By filtering only the routing phases, the discharges in the linear cascaded reservoirs are adjusted to the latest available streamflow measurement, thus in essence fulfilling the task for estimating previous moisture input based on measured streamflow. The updated discharges in the linear cascaded reservoirs therefore reflect the best estimate of previous moisture input.

Exclusion of the moisture input accounting portion significantly simplifies the state space modeling task because the routing phases are readily represented by linear combinations of the states of the system. Variables such as moisture input, soil moisture index, and baseflow infiltration index in the moisture input accounting algorithms are not linearly related to moisture inputs. States derived from these parameters require linearization. Linearization using Taylor Series Expansion (truncating all terms of second and higher orders) itself is an approximation technique which will increase the level the uncertainty in the state space model. The increase in model complexity and decrease in model accuracy further support the decision not to include moisture input accounting in the state space model.

In summary, the following considerations were used in the formation of a state space model for the Mehama Local Basin Model :

1. Moisture input is considered as an input, not a measurement. Moisture input to the watershed will not be filtered as a state. 
2. Soil moisture index and baseflow infiltration index, while they are among the important model variables representing the current condition of the watershed, will not be included as state. Instead, they will be considered as a built-in mathematical component of moisture input.

3. State propagation will be conducted one time step at a time with each time step being 6-hour long.

4. The flows (in CFS) in the cascaded linear reservoirs routing scheme will be filtered. These states completely determine the shape of streamflow hydrograph.

5. The way the SSARR model operates, the states are not measured directly; only the sum of flow of the last reservoir of surface flow, subsurface flow, and baseflow can be measured.

6. The total moisture input of a time step could be calculated separately by comparing the states (discharges in the routing phases) of a time step with its previous time steps. This estimated previous moisture input can be used in re-calculating the moisture splitting functions (SMI, BII, etc.). To simplify the problem, separate calculation for previous total moisture input was not conducted and the moisture splitting functions 
were not re-calculated after the states were filtered. The omission is arguably justified by the high uncertainty in estimating moisture input; while the model forecast is affected heavily by the uncertain moisture input, additional refinement in the moisture splitting functions after each filtering step may bring about only small improvement in forecast accuracy.

The SSARR Mehama Local Basin model in state space form used in this study is given by :

$$
\begin{aligned}
& \mathbf{X}_{\mathrm{t}+1}=\mathbf{T} \mathbf{X}_{\mathrm{t}}+\mathbf{u}_{\mathrm{t}}+\mathbf{w}_{\mathrm{t}} \\
& \mathbf{Z}_{\mathrm{t}+1}=\mathbf{H} \mathbf{X}_{\mathrm{t}+1}+\mathbf{v}_{\mathrm{t}}
\end{aligned}
$$

where $\mathbf{X}$ is state vector containing system states; $\mathbf{u}$ is input vector containing system inputs; $T$ is state transition matrix; $\mathbf{H}$ is system measurement; $\mathbf{Z}$ is system measurement; $\mathbf{w t}_{\mathbf{t}}$ is model noise; and $v_{t}$ is measurement noise. 
The state vector, $\mathbf{X}_{t}$, has the form:

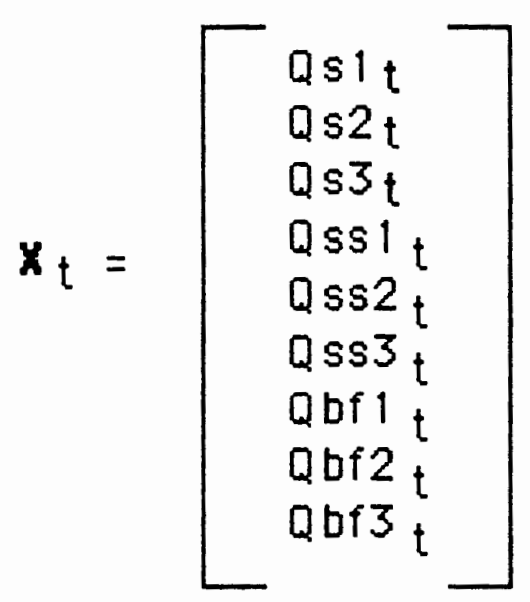

where $\mathbf{X}_{t}$ is the state vector at time step $t$; Qsit is the outflow from the $\mathrm{ith}^{\text {th }}$ reservoir in the linear cascaded reservoir algorithm (LCRA) for the surface flow phase at time step $t$; Qssit is the outflow from the $\mathrm{i}^{\text {th }}$ reservoir in the LCRA for the sub-surface flow phase at time step t; $Q b f l_{t}$ is the outflow from the $i^{\text {th }}$ reservoir in the LCRA for the base flow phase at time step $t$.

The input vector, $\mathbf{u}_{t}$, has nine components: 


$$
u_{t}=\left[\begin{array}{l}
1 s 1 t \\
1 s 2 t \\
1 s 3 t \\
15 s 1 t \\
15 s 2 t \\
15 s 3 t \\
1 b f 1 t \\
1 b f 2 t \\
1 b f 3 t
\end{array}\right]
$$

where $\mathbf{u}_{\mathrm{t}}$ is the input vector at time step $t$; Isit is the moisture input to the $i^{\text {th }}$ reservoir in the linear cascaded reservoir algorithm (LCRA) for the surface flow phase at time step $t$; Issit is the moisture input to the $i^{\text {th }}$ reservoir in the LCRA for the sub-surface flow phase at time step $t$; and $I b f l_{t}$ is the moisture input to the $\mathrm{i}^{\text {th }}$ reservoir in LCRA for the base flow phase at time step t.

The moisture input to surface flow in cubic feet per second for time step $t$ is :

Isi $=2(\mathrm{As} / 2)^{\mathrm{i} I M S}$

where $\mathrm{i}$ signifies the $\mathrm{i}^{\text {th }}$ reservoir in the LCRA; As is

$\mathrm{t} /\left[\mathrm{T}_{\mathrm{S}}+0.5 \mathrm{t}\right] ; \mathrm{t}$ is the length of time step in hour which is 6 hours; $T_{S}$ is 3.5 hours; and IMS is WP $x$ ROP (1.0 - BFP) PS $x$ 
24155. (Refer to Chapter III on the SSARR model for a discussion on the moisture input accounting.)

The Ts for surface flow used in this thesis is different from the value in the RFC version of SSARR Mehama Local Basin Model, of which Ts is 2.5 hours. This change is due to the short time step length. At $\mathrm{t}=6$ hours, As $=1.09$ which is larger 1. In turn, $(1-\mathrm{As})=$ $1-1.09=-0.09$. When $(1-\mathrm{A})$ is negative, the state transition begins to oscillate between the positive and the negative, making the model nonsensical. As a remedy, Ts was changed to 3.5 hours. Thus As = 0.92 and (1-As) is positive.

Moisture input to sub-surface flow in cubic feet per second for time step $t$ is:

Issi $=2($ Ass $/ 2)$ i IMSS

where $i$ signifies the $i^{\text {th }}$ reservoir in the LCRA; Ass is

$t /\left[T_{s s}+0.5 t\right] ; t$ is the length of time step in hours which is 6 hours; $T_{\text {SS }}$ is 8 hours; and IMSS is

WP $\times$ ROP (1.0 - BFP) (1 - PS) x 24155.

Moisture input to baseflow in cubic feet per second for time step $t$ is:

Ibfi $=2(\mathrm{Abf} / 2) \mathrm{i} \mathrm{IMBF}$ 
where $i$ signifies the $i^{\text {th }}$ reservoir in the LCRA; Abf is $t /\left[T_{b f}+0.5 t\right] ; t$ is the length of time step in hour which is 6 hours; Tbf is 60 hours; and IMBF is WP $\times$ ROP $\times$ BFP $\times 24155$.

The state transition matrix takes the form:

$T=\left[\begin{array}{ccccccccc}T_{11} & 0 & 0 & 0 & 0 & 0 & 0 & 0 & 0 \\ T_{21} & T_{22} & 0 & 0 & 0 & 0 & 0 & 0 & 0 \\ T_{31} & T_{32} & T_{33} & 0 & 0 & 0 & 0 & 0 & 0 \\ 0 & 0 & 0 & T_{44} & 0 & 0 & 0 & 0 & 0 \\ 0 & 0 & 0 & T_{54} & T_{55} & 0 & 0 & 0 & 0 \\ 0 & 0 & 0 & T_{64} & T_{65} & T_{66} & 0 & 0 & 0 \\ 0 & 0 & 0 & 0 & 0 & 0 & T_{77} & 0 & 0 \\ 0 & 0 & 0 & 0 & 0 & 0 & T_{87} & T_{88} & 0 \\ 0 & 0 & 0 & 0 & 0 & 0 & T_{97} & T_{98} & T_{99}\end{array}\right.$

where $\mathrm{T}_{11}=\left(1-\mathrm{A}_{\mathrm{S}}\right), \mathrm{T}_{21}=\left(1-\mathrm{A}_{\mathrm{S}}\right), \mathrm{T}_{22}=\left(\mathrm{A}_{\mathrm{S}} / 2\right)\left(2-\mathrm{A}_{\mathrm{S}}\right), \mathrm{T}_{31}=$ $\left(1-A_{S}\right), \quad T_{32}=\left(A_{S} / 2\right)\left(2-A_{S}\right)$, and $\mathrm{T} 33=\left(A_{S} / 2\right)^{2}\left(2-A_{S}\right) ;$

$\mathrm{T}_{44}=\left(1-\mathrm{A}_{\mathrm{SS}}\right), \mathrm{T} 54=\left(1-\mathrm{A}_{\mathrm{SS}}\right)$, T55 = $\left(\mathrm{A}_{\mathrm{Ss}} / 2\right)\left(2-\mathrm{A}_{\mathrm{SS}}\right), \mathrm{T} 64=$ $\left(1-A_{s s}\right), T_{65}=\left(A_{s s} / 2\right)\left(2-A_{S s}\right), \quad$ and $T_{66}=\left(A_{s s} / 2\right)^{2}\left(2-A_{s s}\right)$;

$\mathrm{T} 77=(1-\mathrm{Abf}), \mathrm{T} 87=(1-\mathrm{Abf}), \mathrm{T} 88=\left(\mathrm{Abf}_{\mathrm{bf}}\right)\left(2-\mathrm{Abf}_{\mathrm{b}}\right), \mathrm{T} 97=$ $\left(1-A_{b f}\right)$, T98 $=\left(A_{b f} / 2\right)\left(2-A_{b f}\right)$, and T99 $=\left(A_{b f} / 2\right)^{2}\left(2-A_{b f}\right)$.

Finally, the measurement matrix is given by $\mathbf{H}=\left[\begin{array}{lllllllll}0 & 0 & 1 & 0 & 0 & 1 & 0 & 0 & 1\end{array}\right]$ 


\section{ARTIFICIAL DESIGN OF SYSTEM NOISE COVARIANCE MATRIX}

The analysis of noise was based on the assumption that the "measured" streamflow is the true streamflow. The ultimate task in filtering is therefore to update the model to represent the measured streamflow such that, after filtering the states, the model should yield outflow identical to the measurement. The only remaining question was how the nine states (the nine storages in routing algorithm for the three flow phases) would be adjusted for each measurement in the filtering process ?

The structure of the model covariance directly affects the manner in which each of the states is adjusted by the filter. In other words, the model noise covariance can be designed to control the manner the states are to be adjusted in the filtering process. In the experiment, the design of model covariance was based on several considerations. As discussed earlier, the Kalman filter weights the information from the measurement and the model based on their variances. This weighting algorithm is driven by the relative values of the two variances. By assuming that the measurement is the true value, it is implied that the uncertainty of measurement is very small and the uncertainty in the model is very large. Therefore, at a time step when the moisture input is non-zero, the various routing phases are corrupted by uncertainty in moisture input somewhat evenly. At a time step when the moisture input is zero, the fast responding phases (such as surface flow) are undergoing depletion 
and should have less uncertainty compared to the slower responding phases that are still being excited by previous moisture input. Thus, there should be two model covariance matrices, one for time step with non-zero moisture input and one for time steps with zero moisture input. The magnitude of the variances and covariances in the covariance matrix was assumed to be about 1000 CFS, about one quarter of average streamflow observed in historic data. The uncertainty in model states are lower in magnitude during time steps of zero moisture input.

The model covariance matrix for time step of non-zero moisture input (covariance in units of $10^{6} \mathrm{CFS}^{2}$ ) is:

$$
\begin{array}{lllllllll}
2.4 & 1.8 & 1.3 & 2.1 & 1.5 & 1.2 & 1.7 & 0.9 & 0.7 \\
1.8 & 1.8 & 1.6 & 1.9 & 1.6 & 1.3 & 1.8 & 1.0 & 0.7 \\
1.3 & 1.6 & 1.6 & 1.7 & 1.7 & 1.4 & 1.8 & 1.0 & 0.7 \\
2.1 & 1.9 & 1.7 & 2.3 & 2.0 & 1.7 & 2.4 & 1.4 & 1.0 \\
1.5 & 1.6 & 1.7 & 2.0 & 2.1 & 1.9 & 2.4 & 1.5 & 1.0 \\
1.2 & 1.3 & 1.4 & 1.7 & 1.9 & 1.9 & 2.4 & 1.6 & 1.0 \\
1.7 & 1.8 & 1.8 & 2.4 & 2.4 & 2.4 & 3.5 & 2.6 & 1.9 \\
0.9 & 1.0 & 1.0 & 1.4 & 1.5 & 1.6 & 2.6 & 2.3 & 1.8 \\
0.7 & 0.7 & 0.7 & 1.0 & 1.1 & 1.1 & 1.9 & 1.8 & 1.5
\end{array}
$$

The model covariance matrix for time step of zero moisture input (covariance terms in units of $10^{3}$ CFS $^{2}$; small non-zero covariance terms are shown as 0.0 ) is: 


$\begin{array}{rrrrrrrrr}0.0 & 0.0 & 0.2 & 0.3 & 0.7 & 0.9 & 1.1 & 1.1 & 0.9 \\ 0.1 & 0.7 & 2.1 & 3.4 & 7.5 & 9.4 & 11.3 & 10.1 & 7.5 \\ 0.2 & 2.1 & 7.4 & 11.2 & 26.9 & 36.0 & 40.0 & 31.1 & 19.2 \\ 0.3 & 3.4 & 11.2 & 20.6 & 50.0 & 69.0 & 81.8 & 66.8 & 40.1 \\ 0.7 & 7.5 & 27.0 & 50.0 & 128.2 & 186.6 & 228.5 & 189.4 & 112.6 \\ 0.9 & 9.4 & 36.0 & 69.1 & 186.6 & 285.1 & 370.2 & 318.8 & 194.2 \\ 1.1 & 11.3 & 40.0 & 881.8 & 228.5 & 370.2 & 728.8 & 883.0 & 781.1 \\ 1.1 & 10.1 & 31.1 & 66.8 & 189.4 & 318.8 & 883.0 & 1298.0 & 1348.6 \\ 0.9 & 7.5 & 19.2 & 40.1 & 112.6 & 194.2 & 781.1 & 1348.6 & 1587.8\end{array}$

In reality, this contrast in uncertainty between measurement and model should be less severe due to significant noise in measuring Mehama Local Basin. In that case, after filtering the model would output model streamflow closer but not generally equal to the measured streamflow.

The "measurement" of the Mehama Local Basin is computed by subtracting the routed Big Cliff inflow from the measured Mehama Basin Outflow. This flow of local basin thus consists of two sources of noise. The first source is the noise in the measurement of Mehama outflow and Big Cliff Inflow. The second source is the noise in the routing model (another SSARR model) which is largely controlled by the uncertainty in estimating the precipitation input.

These noises could be mathematically studied and described by statistics. The measurement noise is related to the noise associated with the use of rating table. The noise in routing model is related to the goodness of fit of the particular SSARR model to the respective 
historic data of measured streamflow. Here, to simplify the computational experiment, the measurement of Mehama Local Basin is assumed noiseless.

\section{PERFORMANCE EVALUATION}

The purpose in applying the Kalman filter and a time series model is to achieve better flood forecast accuracy. To assess the usefulness and quality of performance of the new stochastic methods discussed herein, a parallel test was conducted comparing the new and the old methods. The test was based on "root mean square forecast error" which is the square root of the mean of forecast error squared for each of the forecast categories : 6 hour, 12 hour, 18 hour, 24 hour, 48 hour, 72 hour, and 96 hour. A simulated real time operation was conducted based on historic data from the early winter months of 1982 both by the author using the new methods and by the RFC senior hydrologist using the "existing method". The existing method is based on an trial-and-error algorithm which multiplies the storages in the linear cascaded reservoirs by a selection of factors until better fit is achieved. The SSARR Mehama Local Basin Model was updated to measured streamflow every 24 hours.

When the time series model was used, it was operated to enhance the forecast computation externally in the sense that it is not operated as part of the state space model. Hence, the output of the state space model was post-processed by the time series model to generate a streamflow forecast. 
Four simulations were created. The author ran two simulations, one using the Kalman filter without the time series model and one with time series model. The RFC senior hydrologist also ran two simulation, one assuming moisture as all rain and one assuming moisture input as a mixture of rain and snow melt. Historic meteorological data was used to generate the inputs to the model.

After each update, forecast streamflows were computed for the seven categories ( 6 hour, 12 hour, 18 hour, 24 hour, 48 hour, 72 hour, and 96 hour) and root mean square forecast errors were computed, as shown in Figure 12.

The graph shows that the new methods made substantial improvement in the forecast accuracy by almost 1000 CFS for the long lead times such as 24, 48, 72, and 92 hours. Compared with the approximately 4000 CFS mean streamflow of the watershed, this improvement is significant. For the short lead times such as 6, 12, and 18 hours, the new methods did not contribute significant improvement. This may suggest that, given the large uncertainty in the moisture input, SSARR model (alone without the new methods) is performing as well as possible in forecasting for the shorter lead times and no further improvement could be achieved. 


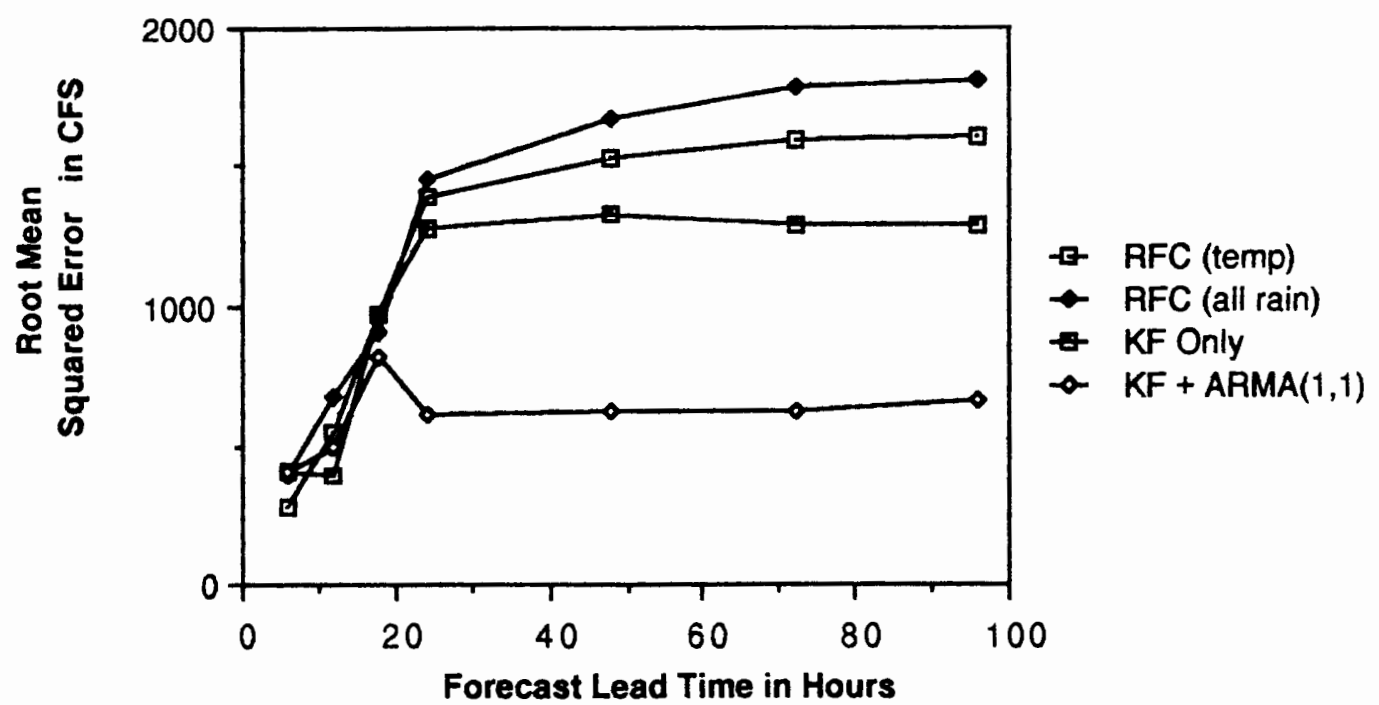

Figure 12. Comparison of performance based on root mean square forecast errors. (Forecast lead times are 6, $12,18,24,48,72$, and 96 hours.)

The graph also shows that forecasts made by the model with or without the new methods could be off by as much as one to two thousands cubic feet per second. This error may seem startling. However one ought to view this error in relation to the mean streamflow of about 4000 CFS and a watershed area of 227 square miles. Due to the large size of the watershed, a small error in moisture input could be translated into large error in streamflow forecast. For example, a moisture input of 1 inch over a 6 hour period is equivalent to 24415 CFS of water influx. For the same 6 hour period, this influx of water is partly stored in the watershed and the rest is discharged as stream runoff. If this 1 inch of moisture input was in error by 0.1 inch, the water influx would be subsequently be off by 2441 CFS. This example illustrates the high 
sensitivity of streamflow to moisture input. The moisture input to the watershed is achieved by estimation which is based on nearby rain gage readings, temperature data, and snow survey data. Other than the simplistic conceptual model structure of SSARR, moisture input is a major source of uncertainty in streamflow forecast. Due to the high sensitivity of modeled streamflow to moisture input, moisture input could well set the upper-limit of achievable forecast accuracy. Every streamflow forecast is generated by some moisture input (zero or non-zero) for future time steps. While the filter can update the model to latest observed streamflow, the streamflow model has no provision for representing the moisture input of the future time steps and is not capable of improving the accuracy of forecast moisture input. 


\section{CHAPTER VIII}

\section{CONCLUSION}

The accuracy of the SSARR Local Basin Model is largely limited by the great uncertainty in the estimated lumped moisture input. Despite the benefits of the stochastic methods, the accuracy of forecast is still dependent on the quality of the forecast model. A Kalman filter improves forecast accuracy by providing a good quality model update from which the model makes the forecast. Therefore, a Kalman filter is not capable of achieving a forecast accuracy higher than the accuracy of the forecast model. However, the use of a filter, such as the Kalman filter used in this thesis, is crucial in updating the model to the latest measurement. Unlike the Kalman filter, a time series model, such as the $\operatorname{ARMA}(1,1)$ model used in this thesis, directly augments the forecast made by the forecast model and could achieve a forecast accuracy higher than the accuracy of the forecast model. This thesis project has illustrated the uses of the Kalman filter and a time series model in the real time operation of a SSARR streamflow forecast model. It shows that the stochastic methods are capable of significantly improving forecast accuracy. 


\section{REFERENCES}

Ambrus, S.Z. and Szollosi-Nagy, "Real time forecasting of water demands using simple adaptive models for various time horizons" Scientific Procedures Applied in the Planning, Design, and Management of Water Resources System (Proceedings of the Hamburg Symposium, August, 1983) IAHS Publ. No. 147, pp 283 - 291.

Anderson, M.G. and Burt T.P. (editors), Hydrological Forecasting, John Wiley and Sons, 1985, pp. 1-12 and pp. 505-558

Bissell, V. C. and Orwig, C. E., Making Observations and Models Agree In Spring Snowmelt Forecasting, Western Snow Conference, Sun Valley, Idaho, 1984 .

Draper, N. and Smith, H. Applied Regression Analysis, Second Edition, John Wiley and Sons, New York, 1981.

Dunne, T., and Leopold, L.B., Water in Environmental Planning, W.H. Freeman and Company, San Francisco, 1978.

Gelb, A. (editor), Applied Optimal Estimation, The MIT Press, Massachusetts Institute of Technology, Cambridge, MA., 1974, pp. $1-179$

Haan, C.T. , Johnson, H.P., and Brakensiek, D.L., ed., (1982) Hydrologic Modeling of Small Watersheds, American Society of Agricultural Engineers, Michigan.

Hains, D., Lecture on Application of Kalman Filter on A RainfallRunoff Model, US Geophysical Union Meeting 1988. Personal communication, 1988.

Jones, G.A. and Koch, R.W., "State Space Approach To Flood Stage Estimation", Proceedings of the ASCE Hydraulics Division Specialty Conference, "Hydrology and Hydraulics in the Small Computer Age", Orlando, FL., August 13-15, 1985. 
Koch, R.W., A Short Course: Optimal Estimation in Hydrology, Department of Civil Engineering, Portland State University, Portland, Oregon 97207, 1985.

Lettenmaier, D. P. and Burges, S., "Use of State Estimation Techniques In Water Resource System Modeling," American Water Resources Association Water Resources Bulletin, Vol 12, No. 1, February 1976.

Maybeck, P.S., Stochastic Models, Estimation, and Control, Volume 1, Mathematics in Science and Engineering Series Volume 141-1, Academic Press, Inc., New York, 1979.

Moll, J.R., "Real Time Flood Forecasting on the River Rhine", Scientific Procedures Applied in the Planning, Design, and Management of Water Resources System (Proceedings of the Hamburg Symposium, August, 1983) IAHS Publ. No. 147, pp 265 - 272.

Moore, S., "Estimating Theory Applications To Design of Water Quality Monitoring Systems", Journal of the Hydraulic Division, ASCE, May, 1973.

Ngan, P. and Russell, S.O., "Example of Flow Forecasting with Kalman Filter", Journal of Hydraulic Engineering, ASCE, Vol 112 No. 9, September, 1986.

Pandit, S.M. and Wu, S.M., Time Series Analysis and System Analysis with Applications, Wiley, New York, 1983.

Reklaitis, G.V., et al, Engineering Optimization, John Wiley and Sons, New York, 1983, p. 22.

Refsgaard, J.C., Rosebjerg, D., and Markussen, L.M., "Application of the Kalman Filter to real time operation and to uncertainty analyses in hydrologic modeling", Scientific Procedures Applied in the Planning, Design, and Management of Water Resources System (Proceedings of the Hamburg Symposium, August, 1983) IAHS Publ. No. 147, pp 273 - 281. 
Salas, J.D., Delleur, J.W., Yevjevich, V., and Lane, W.L., Applied Modeling of Hydrologic Time Series, Water Resources publication, Colorado, 1985.

Schlee, F.H., Standish C.J., and Toda, N.F., "Divergence in the Kalman Filter", AIAA Journal Vol. 5, N0.6, June, 1967, pp 1114-1120.

Shaw, R., The Dripping Faucet as a Model Chaotic System, Aerial Press, Inc. Santa Cruz, 1984.

Szollosi-Nagy, A. "Introductory Remarks on the State Space Modeling of Water Resources Systems," International Institute for Applied Systems Analysis, October, 1976. 


\section{APPENDIX}

\section{DATA USED IN COMPUTATIONAL EXPERIMENT}

\begin{tabular}{|c|c|c|c|c|c|}
\hline Time & $\begin{array}{c}\text { Mehama } \\
\text { Outflow } \\
\text { (CFS) }\end{array}$ & $\begin{array}{l}\text { Routed } \\
\text { Big Cliff } \\
\text { (CFS) }\end{array}$ & $\begin{array}{l}\text { Mehama } \\
\text { Local Basin } \\
\quad(\mathrm{CFS})\end{array}$ & $\begin{array}{c}\text { Moisture } \\
\text { Input } \\
\text { (Inches) }\end{array}$ & Residual \\
\hline 1 & 2819 & 2500 & 319.000 & 0 & 92 \\
\hline 2 & 2844 & 2512 & 332.000 & 0 & 155 \\
\hline 3 & 2703 & 2479 & 224.000 & 0 & 78 \\
\hline 4 & 2730 & 2531 & 199.000 & 0 & 71 \\
\hline 5 & 2820 & 2557 & 263.000 & 0.01 & 147 \\
\hline 6 & 2756 & 2534 & 222.000 & 0.01 & 112 \\
\hline 7 & 2815 & 2530 & 285.000 & 0.02 & 175 \\
\hline 8 & 2780 & 2547 & 233.000 & 0 & 122 \\
\hline 9 & 2736 & 2481 & 255.000 & 0 & 145 \\
\hline 10 & 2705 & 2470 & 235.000 & 0 & 130 \\
\hline 11 & 2829 & 2530 & 299.000 & 0 & 200 \\
\hline 12 & 2776 & 2493 & 283.000 & 0.04 & 186 \\
\hline 13 & 2723 & 2438 & 285.000 & 0.02 & 185 \\
\hline 14 & 2712 & 2486 & 226.000 & 0.01 & 119 \\
\hline 15 & 2789 & 2495 & 294.000 & 0 & 186 \\
\hline 16 & 2750 & 2448 & 302.000 & 0 & 199 \\
\hline 17 & 2710 & 2440 & 270.000 & 0 & 175 \\
\hline 18 & 2671 & 2444 & 227.000 & 0 & 139 \\
\hline 19 & 2814 & 2488 & 326.000 & 0 & 246 \\
\hline 20 & 2820 & 2525 & 295.000 & 0 & 221 \\
\hline 21 & 2729 & 2526 & 203.000 & 0 & 133 \\
\hline 22 & 2755 & 2491 & 264.000 & 0.70 & 125 \\
\hline 23 & 2849 & 2524 & 325.000 & 0.32 & -37 \\
\hline 24 & 3133 & 2612 & 521.000 & 0.06 & -61 \\
\hline 25 & 3221 & 2664 & 557.000 & 0.27 & -139 \\
\hline 26 & 3331 & 2609 & 722.000 & 0.19 & -90 \\
\hline 27 & 3374 & 2548 & 826.000 & 0.07 & -53 \\
\hline 28 & 3700 & 2587 & 1113.000 & 0.09 & 278 \\
\hline 29 & 3700 & 2591 & 1109.000 & 0.13 & 332 \\
\hline 30 & 3817 & 2564 & 1253.000 & 0.06 & 517 \\
\hline 31 & 3766 & 2560 & 1206.000 & 0.01 & 539 \\
\hline
\end{tabular}




\begin{tabular}{|c|c|c|c|c|c|}
\hline 32 & 3737 & 2629 & 1108.000 & 0 & 544 \\
\hline 33 & 3677 & 2696 & 981.000 & 0 & 524 \\
\hline 34 & 3540 & 2644 & 896.000 & 0 & 530 \\
\hline 35 & 3360 & 2631 & 729.000 & 0 & 430 \\
\hline 36 & 3420 & 2695 & 725.000 & 0 & 469 \\
\hline 37 & 3206 & 2613 & 593.000 & 0 & 362 \\
\hline 38 & 3035 & 2496 & 539.000 & 0 & 322 \\
\hline 39 & 3061 & 2596 & 465.000 & 0 & 254 \\
\hline 40 & 3270 & 2762 & 508.000 & 0 & 300 \\
\hline 41 & 3111 & 2719 & 392.000 & 0 & 184 \\
\hline 42 & 2985 & 2592 & 393.000 & 0 & 185 \\
\hline 43 & 2966 & 2553 & 413.000 & 0 & 205 \\
\hline 44 & 3042 & 2602 & 440.000 & 0 & 233 \\
\hline 45 & 3054 & 2662 & 392.000 & 0 & 186 \\
\hline 46 & 2860 & 2622 & 238.000 & 0 & 34 \\
\hline 47 & 2991 & 2585 & 406.000 & 0 & 204 \\
\hline 48 & 3000 & 2589 & 411.000 & 0 & 213 \\
\hline 49 & 3011 & 2603 & 408.000 & 0 & 213 \\
\hline 50 & 2935 & 2592 & 343.000 & 0 & 15 \\
\hline 51 & 2887 & 2529 & 358.000 & 0.23 & 155 \\
\hline 52 & 2850 & 2498 & 352.000 & 0 & 117 \\
\hline 53 & 2879 & 2547 & 332.000 & 0 & 8 \\
\hline 54 & 2887 & 2525 & 362.000 & 0 & 121 \\
\hline 55 & 2647 & 2442 & 205.000 & 0 & -16 \\
\hline 56 & 2750 & 2477 & 273.000 & 0 & 7 \\
\hline 57 & 2925 & 2597 & 328.000 & 0 & 147 \\
\hline 58 & 2863 & 2576 & 287.000 & 0 & 12 \\
\hline 59 & 2786 & 2501 & 285.000 & 0 & 130 \\
\hline 60 & 2782 & 2529 & 253.000 & 0 & 107 \\
\hline 61 & 2857 & 2535 & 322.000 & 0 & 184 \\
\hline 62 & 2844 & 2544 & 300.000 & 0 & 168 \\
\hline 63 & 2914 & 2610 & 304.000 & 0.07 & 174 \\
\hline 64 & 3002 & 2681 & 321.000 & 0.25 & 167 \\
\hline 65 & 2930 & 2625 & 305.000 & 0.08 & 100 \\
\hline 66 & 2926 & 2563 & 363.000 & 0.05 & 117 \\
\hline 67 & 2962 & 2568 & 394.000 & 0.07 & 133 \\
\hline 68 & 3072 & 2566 & 506.000 & 0.07 & 241 \\
\hline 69 & 3069 & 2561 & 508.000 & 0 & 250 \\
\hline 70 & 3034 & 2560 & 474.000 & 0 & 240 \\
\hline 71 & 2946 & 2508 & 438.000 & 0 & 23 \\
\hline 72 & 2878 & 2526 & 352.000 & 0 & 187 \\
\hline 73 & 2991 & 2586 & 405.000 & 0 & 266 \\
\hline 74 & 2930 & 2565 & 365.000 & 0 & 245 \\
\hline 75 & 3180 & 2612 & 568.000 & 0 & 461 \\
\hline 76 & 3090 & 2655 & 435.000 & 0 & 33 \\
\hline 77 & 3005 & 2608 & 397.000 & 0 & 305 \\
\hline 78 & 2944 & 2587 & 357.000 & 0 & 270 \\
\hline 79 & 3011 & 2633 & 378.000 & 0 & 293 \\
\hline
\end{tabular}




\begin{tabular}{|c|c|c|c|c|c|}
\hline 80 & 3040 & 2670 & 370.000 & 0 & 288 \\
\hline 81 & 2985 & 2631 & 354.000 & 0.02 & 274 \\
\hline 82 & 2921 & 2577 & 344.000 & 0.33 & 242 \\
\hline 83 & 3005 & 2574 & 431.000 & 0.15 & 270 \\
\hline 84 & 2990 & 2571 & 419.000 & 0.29 & 168 \\
\hline 85 & 2897 & 2622 & 275.000 & 0.02 & -58 \\
\hline 86 & 3054 & 2660 & 394.000 & 0.21 & 17 \\
\hline 87 & 3164 & 2613 & 551.000 & 0.59 & 39 \\
\hline 88 & 3587 & 2622 & 965.000 & 0.11 & 246 \\
\hline 89 & 3793 & 2625 & 1168.000 & 0.06 & 409 \\
\hline 90 & 3687 & 2625 & 1062.000 & 0.13 & 377 \\
\hline 91 & 3671 & 2647 & 1024.000 & 0.06 & 396 \\
\hline 92 & 3570 & 2620 & 950.000 & 0 & 394 \\
\hline 93 & 3421 & 2576 & 845.000 & 0 & 38 \\
\hline 94 & 3426 & 2583 & 843.000 & 0 & 476 \\
\hline 95 & 3414 & 2637 & 777.000 & 0 & 486 \\
\hline 96 & 3400 & 2662 & 738.000 & 0.08 & 491 \\
\hline 97 & 3354 & 2652 & 702.000 & 0.08 & 459 \\
\hline 98 & 3306 & 2633 & 673.000 & 0.09 & 404 \\
\hline 99 & 3319 & 2643 & 676.000 & 0.01 & 381 \\
\hline 100 & 3342 & 2662 & 680.000 & 0.44 & 317 \\
\hline 101 & 3413 & 2621 & 792.000 & 0.09 & 307 \\
\hline 102 & 3527 & 2602 & 925.000 & 0.22 & 348 \\
\hline 103 & 3774 & 2617 & 1157.000 & 0.26 & 485 \\
\hline 104 & 3986 & 2642 & 1344.000 & 0.07 & 583 \\
\hline 105 & 4343 & 2810 & 1533.000 & 0.05 & 77 \\
\hline 106 & 4544 & 3006 & 1538.000 & 0.07 & 848 \\
\hline 107 & 4313 & 2937 & 1376.000 & 0.01 & 75 \\
\hline 108 & 4099 & 2876 & 1223.000 & 0 & 689 \\
\hline 109 & 4000 & 2980 & 1020.000 & 0 & 570 \\
\hline 110 & 4055 & 3039 & 1016.000 & 0.33 & 580 \\
\hline 111 & 4242 & 3085 & 1157.000 & 0.47 & 547 \\
\hline 112 & 5962 & 3059 & 2903.000 & 0.55 & 1876 \\
\hline 113 & 11505 & 3006 & 8499.000 & 0.51 & 6919 \\
\hline 114 & 11423 & 2156 & 9267.000 & 0.62 & 709 \\
\hline 115 & 7584 & 1427 & 6157.000 & 0.08 & 369 \\
\hline 116 & 6595 & 1845 & 4750.000 & 0 & $260^{\circ}$ \\
\hline 117 & 6350 & 2673 & 3677.000 & 0 & 205 \\
\hline 118 & 5731 & 3109 & 2622.000 & 0 & 1362 \\
\hline 119 & 5433 & 3235 & 2198.000 & 0 & 1217 \\
\hline 120 & 4955 & 3160 & 1795.000 & 0 & 101 \\
\hline 121 & 4389 & 3011 & 1378.000 & 0 & 728 \\
\hline 122 & 4769 & 3110 & 1659.000 & 0 & 1087 \\
\hline 123 & 4677 & 3255 & 1422.000 & 0 & 89 \\
\hline 124 & 4450 & 3189 & 1261.000 & 0.07 & 740 \\
\hline 125 & 4209 & 3072 & 1137.000 & 0.07 & 58 \\
\hline 126 & 4084 & 3007 & 1077.000 & 0.11 & 46 \\
\hline
\end{tabular}




\begin{tabular}{|c|c|c|c|c|c|}
\hline 127 & 4145 & 3000 & 1145.000 & 0 & 474 \\
\hline 128 & 4160 & 3044 & 1116.000 & 0 & 435 \\
\hline 129 & 3943 & 3019 & 924.000 & 0 & 274 \\
\hline 130 & 3820 & 2939 & 881.000 & 0 & 271 \\
\hline 131 & 3836 & 2948 & 888.000 & 0 & 317 \\
\hline 132 & 3830 & 2968 & 862.000 & 0 & 321 \\
\hline 133 & 3787 & 2952 & 835.000 & 0 & 318 \\
\hline 134 & 3983 & 3096 & 887.000 & 0 & 388 \\
\hline 135 & 3739 & 3146 & 593.000 & 0 & 109 \\
\hline 136 & 3780 & 3056 & 724.000 & 0.02 & 252 \\
\hline 137 & 3652 & 3023 & 629.000 & 0.06 & 160 \\
\hline 138 & 3696 & 3011 & 685.000 & 0.29 & 176 \\
\hline 139 & 3689 & 3006 & 683.000 & 0.37 & 24 \\
\hline 140 & 3742 & 2978 & 764.000 & 0.11 & -93 \\
\hline 141 & 3718 & 3073 & 645.000 & 0 & -279 \\
\hline 142 & 3689 & 3098 & 591.000 & 0.08 & -280 \\
\hline 143 & 3933 & 3006 & 927.000 & 0.19 & 87 \\
\hline 144 & 5517 & 2982 & 2535.000 & 0 & 1698 \\
\hline 145 & 5419 & 2989 & 2430.000 & 0.01 & 1649 \\
\hline 146 & 5124 & 3003 & 2121.000 & 0.02 & 1435 \\
\hline 147 & 4986 & 3067 & 1919.000 & 0.01 & 1316 \\
\hline 148 & 4953 & 3133 & 1820.000 & 0 & 1287 \\
\hline 149 & 4705 & 3113 & 1592.000 & 0 & 1117 \\
\hline 150 & 4489 & 3039 & 1450.000 & 0 & 1021 \\
\hline 151 & 4383 & 2994 & 1389.000 & 0 & 994 \\
\hline 152 & 4340 & 2990 & 1350.000 & 0 & 982 \\
\hline 153 & 4236 & 3043 & 1193.000 & 0 & 844 \\
\hline 154 & 4180 & 3077 & 1103.000 & 0 & 769 \\
\hline 155 & 4074 & 3026 & 1048.000 & 0 & 726 \\
\hline 156 & 4000 & 2993 & 1007.000 & 0 & 696 \\
\hline 157 & 4740 & 3385 & 1355.000 & 0 & 1054 \\
\hline 158 & 4878 & 3830 & 1048.000 & 0 & 757 \\
\hline 159 & 4695 & 3842 & 853.000 & 0 & 572 \\
\hline 160 & 4661 & 3845 & 816.000 & 0 & 545 \\
\hline 161 & 4754 & 3850 & 904.000 & 0 & 642 \\
\hline 162 & 4571 & 3832 & 739.000 & 0 & 487 \\
\hline 163 & 4467 & 3765 & 702.000 & 0 & 460 \\
\hline 164 & 4603 & 3820 & 783.000 & 0 & 551 \\
\hline 165 & 4439 & 3848 & 591.000 & 0 & 368 \\
\hline 166 & 4609 & 3845 & 764.000 & 0 & 551 \\
\hline 167 & 4348 & 3946 & 402.000 & 0 & 197 \\
\hline 168 & 4354 & 3960 & 394.000 & 0 & 199 \\
\hline 169 & 4313 & 3881 & 432.000 & 0 & 246 \\
\hline 170 & 4356 & 3870 & 486.000 & 0 & 309 \\
\hline 171 & 4361 & 3948 & 413.000 & 0 & 244 \\
\hline 172 & 4410 & 3913 & 497.000 & 0.01 & 337 \\
\hline 173 & 4124 & 3771 & 353.000 & 0 & 199 \\
\hline 174 & 4322 & 3824 & 498.000 & 0 & 351 \\
\hline
\end{tabular}




\begin{tabular}{|c|c|c|c|c|c|}
\hline 175 & 4489 & 4001 & 488.000 & 0 & 349 \\
\hline 176 & 4290 & 3936 & 354.000 & 0 & 222 \\
\hline 177 & 4139 & 3819 & 320.000 & 0 & 196 \\
\hline 178 & 4340 & 3888 & 452.000 & 0 & 335 \\
\hline 179 & 4440 & 3974 & 466.000 & 0 & 356 \\
\hline 180 & 4525 & 3975 & 550.000 & 0 & 446 \\
\hline 181 & 4278 & 3896 & 382.000 & 0 & 285 \\
\hline 182 & 4389 & 3872 & 517.000 & 0 & 425 \\
\hline 183 & 4524 & 3962 & 562.000 & 0 & 476 \\
\hline 184 & 4595 & 4021 & 574.000 & 0 & 493 \\
\hline 185 & 4137 & 3751 & 386.000 & 0.11 & 303 \\
\hline 186 & 3981 & 3450 & 531.000 & 0.23 & 416 \\
\hline 187 & 4022 & 3570 & 452.000 & 0.07 & 280 \\
\hline 188 & 4230 & 3681 & 549.000 & 0.31 & 297 \\
\hline 189 & 4360 & 3585 & 775.000 & 0.69 & 250 \\
\hline 190 & 5307 & 3511 & 1796.000 & 0.23 & 877 \\
\hline 191 & 5610 & 3596 & 2014.000 & 0.23 & 920 \\
\hline 192 & 5951 & 3706 & 2245.000 & 0 & 1184 \\
\hline 193 & 6293 & 3637 & 2656.000 & 0.96 & 1306 \\
\hline 194 & 6861 & 3639 & 3222.000 & 0.35 & 1222 \\
\hline 195 & 7160 & 3786 & 3374.000 & 0.38 & 1041 \\
\hline 196 & 7014 & 3837 & 3177.000 & 0.53 & 604 \\
\hline 197 & 6593 & 3845 & 2748.000 & 0.40 & -260 \\
\hline 198 & 6739 & 3919 & 2820.000 & 0.20 & -276 \\
\hline 199 & 7110 & 4226 & 2884.000 & 0.13 & 148 \\
\hline 200 & 6784 & 4114 & 2670.000 & 0.13 & 341 \\
\hline 201 & 6732 & 4319 & 2413.000 & 0.28 & 228 \\
\hline 202 & 7128 & 4908 & 2220.000 & 0.07 & 48 \\
\hline 203 & 6883 & 4847 & 2036.000 & 0.20 & -18 \\
\hline 204 & 6888 & 4934 & 1954.000 & 0.29 & -142 \\
\hline 205 & 6800 & 4973 & 1827.000 & 0.06 & -393 \\
\hline 206 & 6459 & 4797 & 1662.000 & 0 & -364 \\
\hline 207 & 6524 & 4762 & 1762.000 & 0.01 & 68 \\
\hline 208 & 6275 & 4900 & 1375.000 & 0 & -66 \\
\hline 209 & 6489 & 4999 & 1490.000 & 0 & 244 \\
\hline 210 & 6219 & 4845 & 1374.000 & 0 & 270 \\
\hline 211 & 6164 & 4639 & 1525.000 & 0 & 513 \\
\hline 212 & 5880 & 4630 & 1250.000 & 0 & 292 \\
\hline 213 & 6115 & 4858 & 1257.000 & 0 & 328 \\
\hline 214 & 6109 & 4955 & 1154.000 & 0 & 241 \\
\hline 215 & 5949 & 4838 & 1111.000 & 0 & 204 \\
\hline 216 & 5875 & 4690 & 1185.000 & 0 & 283 \\
\hline 217 & 5700 & 4755 & 945.000 & 0 & 48 \\
\hline 218 & 5960 & 4928 & 1032.000 & 0 & 142 \\
\hline 219 & 5939 & 4982 & 957.000 & 0 & 76 \\
\hline 220 & 5700 & 4713 & 987.000 & 0 & 117 \\
\hline 221 & 5544 & 4453 & 1091.000 & 0 & 236 \\
\hline 222 & 5098 & 4370 & 728.000 & 0 & -111 \\
\hline
\end{tabular}




\begin{tabular}{|c|c|c|c|c|c|}
\hline 223 & 5112 & 4226 & 886.000 & 0 & 64 \\
\hline 224 & 5166 & 4262 & 904.000 & 0 & 105 \\
\hline 225 & 5118 & 4415 & 703.000 & 0 & -75 \\
\hline 226 & 5189 & 4369 & 820.000 & 0 & 6 \\
\hline 227 & 5016 & 4212 & 804.000 & 0.01 & 7 \\
\hline 228 & 5075 & 4242 & 833.000 & 0.01 & 12 \\
\hline 229 & 5058 & 4236 & 822.000 & 0 & 13 \\
\hline 230 & 5210 & 4179 & 1031.000 & 0.29 & 32 \\
\hline 231 & 5292 & 4198 & 1094.000 & 0.17 & 29 \\
\hline 232 & 5655 & 4312 & 1343.000 & 0.71 & 11 \\
\hline 233 & 5844 & 4311 & 1533.000 & 0.32 & -41 \\
\hline 234 & 6333 & 4329 & 2004.000 & 0.08 & -24 \\
\hline 235 & 6551 & 4492 & 2059.000 & 0.04 & 6 \\
\hline 236 & 6522 & 4482 & 2040.000 & 0.19 & 28 \\
\hline 237 & 6296 & 4415 & 1881.000 & 0.45 & -9 \\
\hline 238 & 7002 & 4452 & 2550.000 & 0.37 & -2 \\
\hline 239 & 7110 & 4423 & 2687.000 & 0.54 & -56 \\
\hline 240 & 7139 & 4327 & 2812.000 & 0.57 & -124 \\
\hline 241 & 7596 & 4588 & 3008.000 & 0.44 & -1755 \\
\hline 242 & 7962 & 5042 & 2920.000 & 0.02 & -172 \\
\hline 243 & 7499 & 5051 & 2448.000 & 0.06 & -1277 \\
\hline 244 & 7570 & 4965 & 2605.000 & 0.08 & -28 \\
\hline 245 & 7517 & 4988 & 2529.000 & 0.06 & 9 \\
\hline 246 & 7397 & 4934 & 2463.000 & 0.06 & 33 \\
\hline 247 & 7289 & 5005 & 2284.000 & 0 & 39 \\
\hline 248 & 7203 & 5170 & 2033.000 & 0 & 37 \\
\hline 249 & 7128 & 5293 & 1835.000 & 0.11 & 31 \\
\hline 250 & 7008 & 5220 & 1788.000 & 0.61 & -21 \\
\hline 251 & 8203 & 5163 & 3040.000 & 0.81 & -58 \\
\hline 252 & 4347 & 3282 & 1065.000 & 0.64 & 550 \\
\hline 253 & 4200 & 1339 & 2861.000 & 0.34 & 644 \\
\hline 254 & 11003 & 1780 & 9223.000 & 0.21 & 317 \\
\hline 255 & 11592 & 2966 & 8626.000 & 0.82 & 237 \\
\hline 256 & 4355 & 2384 & 1971.000 & 1.32 & 293 \\
\hline 257 & 3956 & 1302 & 2654.000 & 0.21 & 135 \\
\hline 258 & 9724 & 1404 & 8320.000 & 0.08 & -111 \\
\hline 259 & 10411 & 2966 & 7445.000 & 0 & 91 \\
\hline 260 & 10934 & 4930 & 6004.000 & 0 & 100 \\
\hline 261 & 9611 & 5006 & 4605.000 & 0 & 57 \\
\hline 262 & 8846 & 4718 & 4128.000 & 0.09 & 69 \\
\hline 263 & 9038 & 4821 & 4217.000 & 0.80 & -2 \\
\hline 264 & 10775 & 4830 & 5945.000 & 0.48 & -38 \\
\hline 265 & 12246 & 4895 & 7351.000 & 0.17 & 23 \\
\hline 266 & 11195 & 4933 & 6262.000 & 0.16 & -11 \\
\hline 267 & 10320 & 5118 & 5202.000 & 0.06 & -43 \\
\hline 268 & 9912 & 5328 & 4584.000 & 0 & -40 \\
\hline 269 & 9032 & 5109 & 3923.000 & 0 & -43 \\
\hline 270 & 8779 & 5180 & 3599.000 & 0 & -258 \\
\hline
\end{tabular}




\begin{tabular}{|c|c|c|c|c|c|}
\hline 271 & 8536 & 5364 & 3172.000 & 0 & -309 \\
\hline 272 & 8181 & 5200 & 2981.000 & 0 & -238 \\
\hline 273 & 7890 & 5011 & 2879.000 & 0 & -169 \\
\hline 274 & 7788 & 5131 & 2657.000 & 0 & -282 \\
\hline 275 & 7648 & 5215 & 2433.000 & 0 & -435 \\
\hline 276 & 7457 & 5088 & 2369.000 & 0 & -447 \\
\hline 277 & 7260 & 5136 & 2124.000 & 0 & -649 \\
\hline 278 & 7328 & 5267 & 2061.000 & 0 & -669 \\
\hline 279 & 7266 & 5251 & 2015.000 & 0 & -670 \\
\hline 280 & 7200 & 5250 & 1950.000 & 0 & -685 \\
\hline 281 & 7140 & 5208 & 1932.000 & 0 & -648 \\
\hline 282 & 7068 & 5219 & 1849.000 & 0 & -672 \\
\hline 283 & 6990 & 5159 & 1831.000 & 0 & -625 \\
\hline 284 & 6676 & 5202 & 1474.000 & 0 & -914 \\
\hline 285 & 6946 & 5445 & 1501.000 & 0 & -815 \\
\hline 286 & 6889 & 5484 & 1405.000 & 0 & -836 \\
\hline 287 & 6513 & 5291 & 1222.000 & 0 & -943 \\
\hline 288 & 6425 & 4931 & 1494.000 & 0 & -593 \\
\hline 289 & 5871 & 4723 & 1148.000 & 0 & -860 \\
\hline 290 & 6051 & 4789 & 1262.000 & 0.14 & -696 \\
\hline 291 & 5830 & 4804 & 1026.000 & 0.60 & -1260 \\
\hline 292 & 6150 & 4740 & 1410.000 & 0.21 & -1563 \\
\hline 293 & 5932 & 4688 & 1244.000 & 0.14 & -2061 \\
\hline 294 & 6220 & 4718 & 1502.000 & 0.02 & -1651 \\
\hline 295 & 6108 & 4757 & 1351.000 & 0.06 & -1507 \\
\hline 296 & 6155 & 4732 & 1423.000 & 0.27 & -1399 \\
\hline 297 & 6509 & 4898 & 1611.000 & 0.23 & -1566 \\
\hline 298 & 6623 & 4868 & 1755.000 & 0.66 & -2327 \\
\hline 299 & 6900 & 4785 & 2115.000 & 0.24 & -3045 \\
\hline 300 & 7513 & 4981 & 2532.000 & 0.23 & -2742 \\
\hline 301 & 7486 & 5075 & 2411.000 & 0.56 & -2969 \\
\hline 302 & 8561 & 5089 & 3472.000 & 0.40 & -2760 \\
\hline 303 & 10114 & 5207 & 4907.000 & 1.05 & -3093 \\
\hline 304 & 11430 & 5366 & 6064.000 & 0.71 & -4491 \\
\hline 305 & 9628 & 3362 & 6266.000 & 1.20 & -6699 \\
\hline 306 & 8211 & 1272 & 6939.000 & 0.17 & -6824 \\
\hline 307 & 7140 & 1034 & 6106.000 & 0.06 & -4934 \\
\hline 308 & 6503 & 1007 & 5496.000 & 0.54 & -2976 \\
\hline 309 & 6097 & 1014 & 5083.000 & 0.52 & -3876 \\
\hline 310 & 5769 & 1020 & 4749.000 & 0.34 & -4832 \\
\hline 311 & 5471 & 1006 & 4465.000 & 0.31 & -4675 \\
\hline 312 & 5210 & 1000 & 4210.000 & 0.27 & -4352 \\
\hline 313 & 4660 & 1000 & 3660.000 & 0.06 & -4174 \\
\hline 314 & 4286 & 2025 & 2261.000 & 0.06 & -4453 \\
\hline 315 & 8494 & 4370 & 4124.000 & 0.33 & -210 \\
\hline 316 & 8298 & 5100 & 3198.000 & 0.09 & -3216 \\
\hline 317 & 8229 & 5064 & 3165.000 & 0.06 & -2915 \\
\hline 318 & 8211 & 6061 & 2150.000 & 0 & -3269 \\
\hline
\end{tabular}




$\begin{array}{rrr}319 & 9886 & 7034 \\ 320 & 9940 & 7128 \\ 321 & 9778 & 7086 \\ 322 & 9948 & 7032 \\ 323 & 10064 & 7035 \\ 324 & 10124 & 7088 \\ 325 & 10432 & 7109 \\ 326 & 11382 & 7638 \\ 327 & 12188 & 8356 \\ 328 & 11975 & 8478 \\ 329 & 11917 & 8441 \\ 330 & 11878 & 8582 \\ 331 & 11608 & 8614 \\ 332 & 11470 & 8399 \\ 333 & 11598 & 8429 \\ 334 & 11425 & 8694 \\ 335 & 11391 & 8901 \\ 336 & 11346 & 9003 \\ 337 & 9106 & 8081 \\ 338 & 9049 & 7161 \\ 339 & 9050 & 7164 \\ 340 & 8813 & 7121 \\ 341 & 8698 & 7013 \\ 342 & 8971 & 6957 \\ 343 & 8864 & 7114 \\ 344 & 8840 & 7241 \\ 345 & 8840 & 7211 \\ 346 & 8861 & 7312 \\ 347 & 8729 & 7315 \\ 348 & 8701 & 7233 \\ 349 & 8474 & 7186 \\ 350 & 7231 & 6211 \\ 351 & 6188 & 5012 \\ 352 & 6379 & 4768 \\ 353 & 6230 & 4825 \\ 354 & 6170 & 4839 \\ 355 & 6157 & 4873 \\ 356 & 6150 & 5025 \\ 357 & 6387 & 5091 \\ 358 & 6040 & 4949 \\ 359 & 6015 & 4809 \\ 360 & 6117 & 4791 \\ 361 & 6003 & 4883 \\ 362 & 6200 & 4923 \\ 363 & 6120 & 4902 \\ 364 & 6195 & 4727 \\ 365 & 3988 & 3923 \\ 366 & 4249 & 3239\end{array}$

$\begin{array}{rlr}2852.000 & 0.06 & -2132 \\ 2812.000 & 0.33 & -2331 \\ 2692.000 & 0.85 & -4375 \\ 2916.000 & 0.31 & -6508 \\ 3029.000 & 0.09 & -6256 \\ 3036.000 & 0.16 & -4837 \\ 3323.000 & 0.47 & -4470 \\ 3744.000 & 0.09 & -4519 \\ 3832.000 & 0.27 & -4147 \\ 3497.000 & 0.35 & -4466 \\ 3476.000 & 0.78 & -6000 \\ 3296.000 & 0.49 & -8231 \\ 2994.000 & 0.01 & -8373 \\ 3071.000 & 0.20 & -6381 \\ 3169.000 & 0.22 & -5271 \\ 2731.000 & 0.20 & -5509 \\ 2490.000 & 0.07 & -5323 \\ 2343.000 & 0 & -4672 \\ 1025.000 & 0 & -5175 \\ 1888.000 & 0 & -3704 \\ 1886.000 & 0 & -3252 \\ 1692.000 & 0 & -3113 \\ 1685.000 & 0 & -2881 \\ 2014.000 & 0 & -2379 \\ 1750.000 & 0 & -2512 \\ 1599.000 & 0 & -2555 \\ 1629.000 & 0.19 & -2582 \\ 1549.000 & 0 & -2834 \\ 1414.000 & 0 & -2917 \\ 1468.000 & 0 & -2649 \\ 1288.000 & 0 & -2637 \\ 1020.000 & 0 & -2722 \\ 1176.000 & 0 & -2396 \\ 1611.000 & 0 & -1808 \\ 1405.000 & 0 & -1873 \\ 1331.000 & 0 & -1815 \\ 1284.000 & 0 & -1736 \\ 1125.000 & 0 & -1774 \\ 1296.000 & 0 & -1485 \\ 1091.000 & 0 & -1572 \\ 1206.000 & 0 & -1342 \\ 1326.000 & 0 & -1108 \\ 1120.000 & 0 & -1205 \\ 1277.000 & 0 & -939 \\ 1218.000 & 0 & -892 \\ 1468.000 & 0 & -538 \\ 65.000 & 0 & -1841 \\ 1010.000 & 0 & -802\end{array}$


134

$\begin{array}{rrrrrr}367 & 4258 & 3098 & 1160.000 & 0 & -558 \\ 368 & 3910 & 2987 & 923.000 & 0 & -702\end{array}$

